\title{
A Green's-Function Approach to Exchange Spin Coupling As a New Tool for Quantum Chemistry - Supporting Information
}

Torben Steenbock, Jos Tasche ${ }^{\dagger}$, Alexander I. Lichtenstein ${ }^{\ddagger}$, Carmen Herrmann ${ }^{\star}$

Department of Chemistry, University of Hamburg, Martin-Luther-King-Platz 6, Hamburg , 20146 Germany

$\dagger$ Department of Chemistry, Durham University, South Road, Durham, United Kingdom

$\ddagger$ Department of Physics, University Hamburg, Jungiusstrasse 9, Hamburg, 20355 Germany

* To whom correspondence should be addressed

email: carmen.herrmann@chemie.uni-hamburg.de; TEL: +49-40-42838-6934

Date: November 9th, 2015

Status: Final Version 


\section{The dihydrogen molecule}

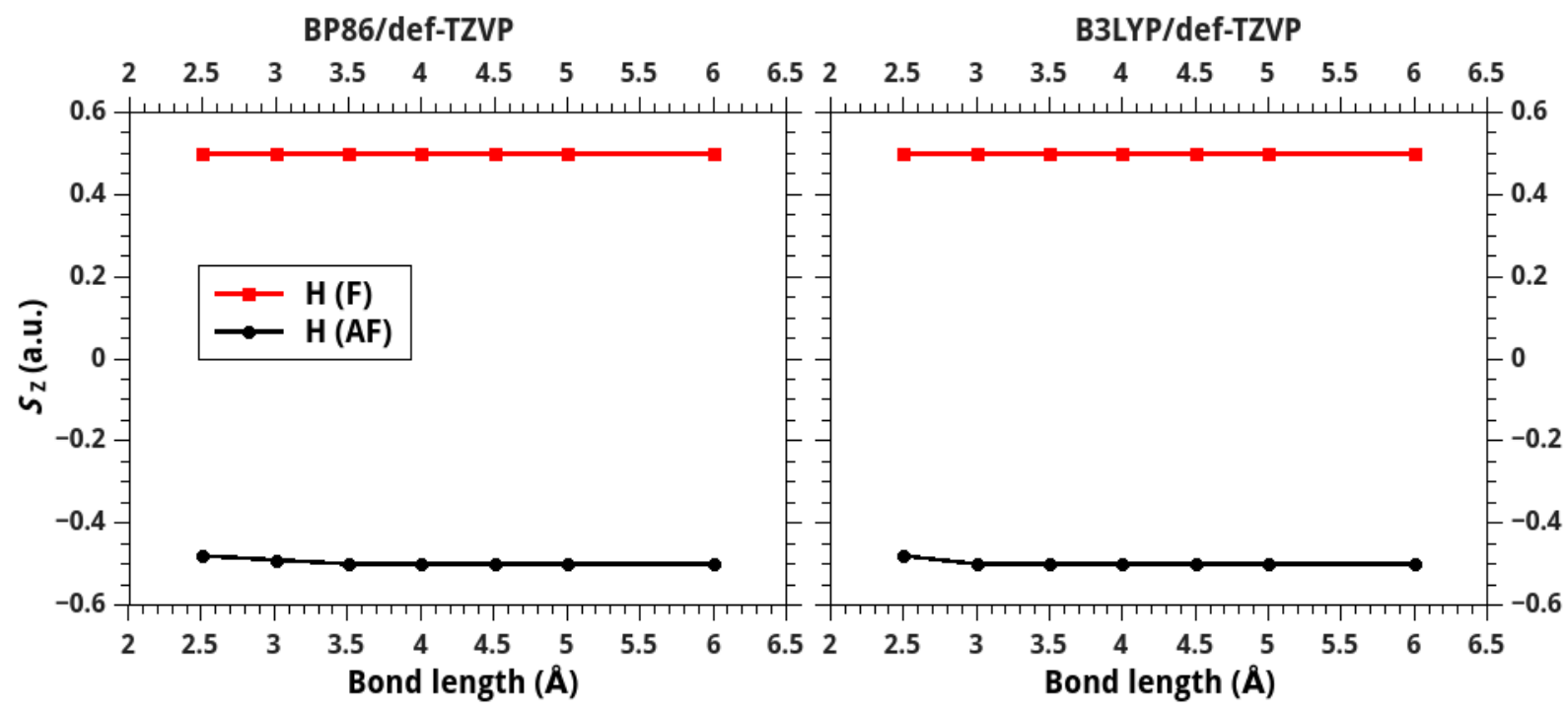

Figure S1: Local spins for the dihydrogen molecule obtained by a Mulliken population analysis using BP86 (left-hand site) and B3LYP (right-hand site). Basis set: def-TZVP. 


\section{$2 \quad \mathrm{H}-\mathrm{He}-\mathrm{H}$ model system}
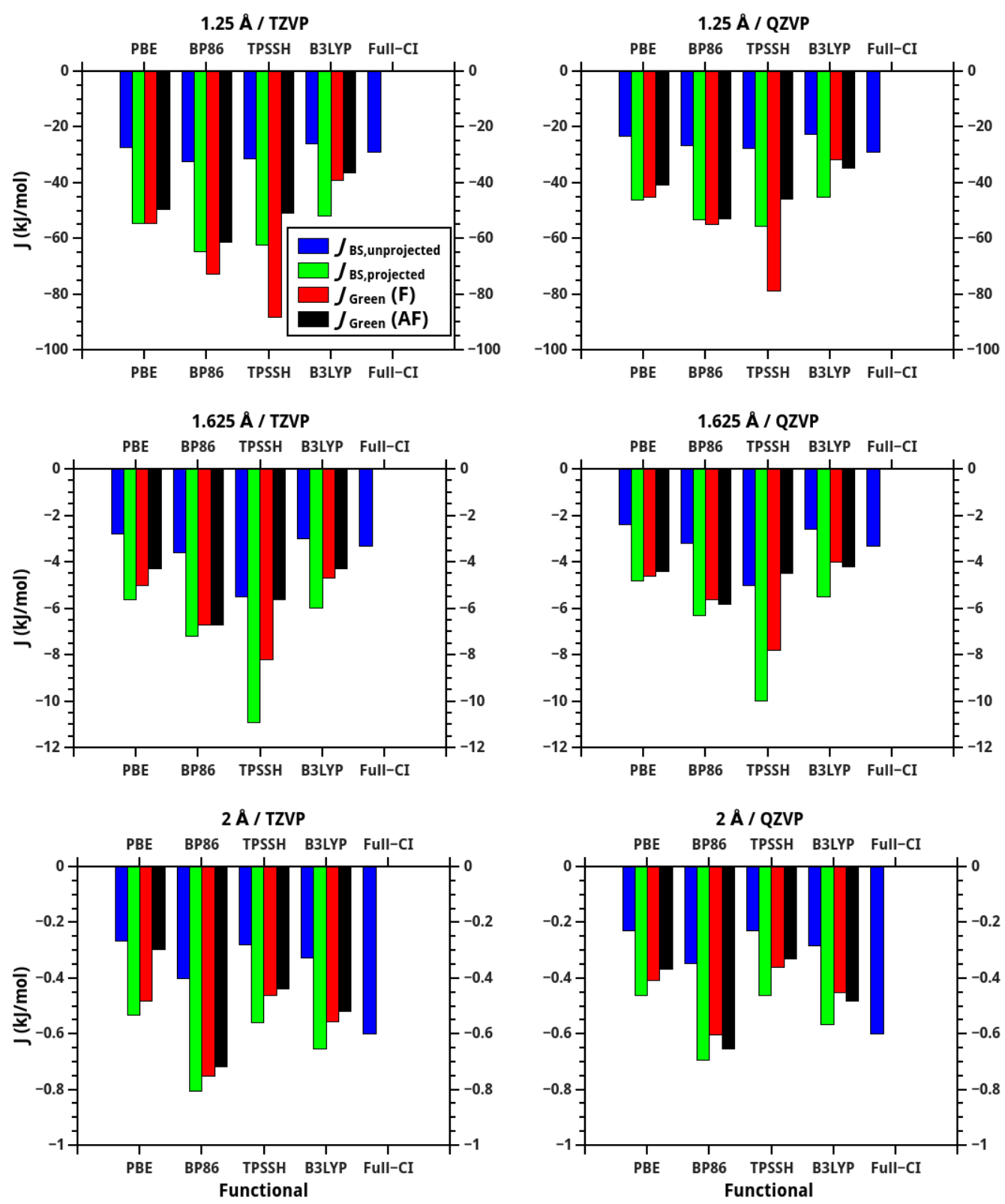

Figure S2: Coupling constants calculated with different exchange-correlation functionals using the def-TZVP and the def-QZVP basis set. 


\section{$3 \quad$ Larger systems with localized spins}

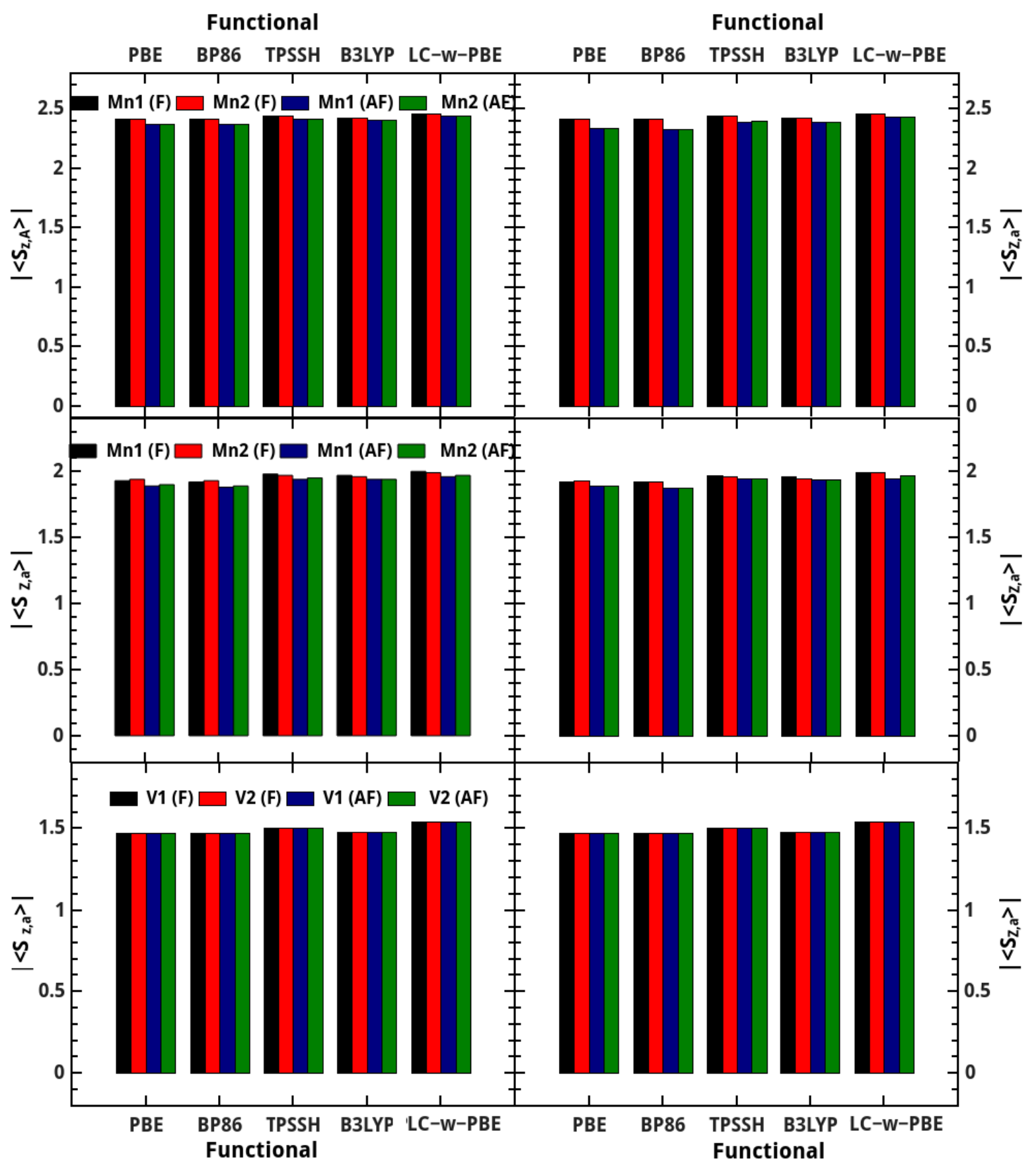

Figure S3: Local spins for $\left[\mathrm{Mn}_{2}(\mathrm{Ac})_{4}\left(\mathrm{H}_{2} \mathrm{O}\right)_{2}\right]$ (upper row), $\left[\mathrm{Mn}_{2}(\mu-\mathrm{OMe})_{2}(\mathrm{HL})_{4}\right]$ (middle row) and $\left[\mathrm{V}_{2}\left(\eta^{5}-\mathrm{Cp}\right)_{2}\left(\mu-\eta^{5}-1,8-\mathrm{DCN}\right)\right]$ (lower row) calculated with different exchange-correlation functionals for the high-spin structure (left column) and the BS structure (right column). 
Table S1: Mulliken local spins for the both spin-state structures and both spin states of calculated with different exchange-correlation functionals for the $\left[\mathrm{Mn}_{2}(\mathrm{Ac})_{4}\left(\mathrm{H}_{2} \mathrm{O}\right)_{2}\right]$ complex. The values are given in a.u.. Basis set: def-TZVP.

\begin{tabular}{|c|c|c|c|c|}
\hline \multicolumn{5}{|c|}{ high-spin structure } \\
\hline Functional & $\left|\left\langle\hat{S}_{z, A}\right\rangle\right|(\mathrm{Mn} 1, \mathrm{~F})$ & $\left|\left\langle\hat{S}_{z, A}\right\rangle\right|(\mathrm{Mn} 2, \mathrm{~F})$ & $\left|\left\langle\hat{S}_{z, A}\right\rangle\right|(\mathrm{Mn} 1, \mathrm{AF})$ & $\left|\left\langle\hat{S}_{z, A}\right\rangle\right|(\mathrm{Mn} 2, \mathrm{AF})$ \\
\hline PBE & 2.415 & 2.416 & 2.375 & 2.377 \\
\hline BP86 & 2.416 & 2.418 & 2.375 & 2.377 \\
\hline TPSSH & 2.444 & 2.446 & 2.420 & 2.421 \\
\hline B3LYP & 2.424 & 2.426 & 2.408 & 2.409 \\
\hline LC- $\omega$ PBE & 2.459 & 2.460 & 2.447 & 2.447 \\
\hline \multicolumn{5}{|c|}{ BS structure } \\
\hline $\mathrm{PBE}$ & 2.418 & 2.419 & 2.335 & 2.336 \\
\hline $\mathrm{BP} 86$ & 2.418 & 2.420 & 2.332 & 2.333 \\
\hline TPSSH & 2.446 & 2.447 & 2.394 & 2.395 \\
\hline B3LYP & 2.425 & 2.425 & 2.387 & 2.387 \\
\hline $\mathrm{LC}-\omega \mathrm{PBE}$ & 2.459 & 2.460 & 2.431 & 2.431 \\
\hline
\end{tabular}


Table S2: Mulliken local spins for the both spin-state structures and both spin states of calculated with different exchange-correlation functionals for the $\left[\mathrm{Mn}_{2}(\mu-\mathrm{OMe})_{2}(\mathrm{HL})_{4}\right]$ complex. The values are given in a.u.. Basis set: def-TZVP.

\begin{tabular}{|c|c|c|c|c|}
\hline \multicolumn{5}{|c|}{ high-spin structure } \\
\hline Functional & $\left|\left\langle\hat{S}_{z, A}\right\rangle\right|(\mathrm{Mn} 1, \mathrm{~F})$ & $\left|\left\langle\hat{S}_{z, A}\right\rangle\right|(\mathrm{Mn} 2, \mathrm{~F})$ & $\left|\left\langle\hat{S}_{z, A}\right\rangle\right|(\mathrm{Mn} 1, \mathrm{AF})$ & $\left|\left\langle\hat{S}_{z, A}\right\rangle\right|(\mathrm{Mn} 2, \mathrm{AF})$ \\
\hline PBE & 1.927 & 1.939 & 1.889 & 1.898 \\
\hline BP86 & 1.918 & 1.929 & 1.877 & 1.886 \\
\hline TPSSH & 1.977 & 1.966 & 1.947 & 1.953 \\
\hline B3LYP & 1.965 & 1.955 & 1.940 & 1.944 \\
\hline $\mathrm{LC}-\omega \mathrm{PBE}$ & 1.999 & 1.990 & 1.956 & 1.973 \\
\hline \multicolumn{5}{|c|}{ BS structure } \\
\hline PBE & 1.925 & 1.927 & 1.888 & 1.894 \\
\hline BP86 & 1.916 & 1.917 & 1.876 & 1.882 \\
\hline TPSSH & 1.970 & 1.961 & 1.946 & 1.951 \\
\hline B3LYP & 1.958 & 1.953 & 1.939 & 1.943 \\
\hline $\mathrm{LC}-\omega \mathrm{PBE}$ & 1.993 & 1.990 & 1.985 & 1.982 \\
\hline
\end{tabular}


Table S3: Mulliken local spins for the both spin-state structures and both spin states of calculated with different exchange-correlation functionals for the $\left[\mathrm{V}_{2}\left(\eta^{5}-\mathrm{Cp}\right)_{2}\left(\mu-\eta^{5}-1,8-\mathrm{DCN}\right)\right]$ complex. The values are given in a.u.. Basis set: def-TZVP.

\begin{tabular}{|c|c|c|c|c|}
\hline \multicolumn{5}{|c|}{ high-spin structure } \\
\hline Functional & $\left|\left\langle\hat{S}_{z, A}\right\rangle\right|(\mathrm{V} 1, \mathrm{~F})$ & $\left|\left\langle\hat{S}_{z, A}\right\rangle\right|(\mathrm{V} 2, \mathrm{~F})$ & $\left|\left\langle\hat{S}_{z, A}\right\rangle\right|(\mathrm{V} 1, \mathrm{AF})$ & $\left|\left\langle\hat{S}_{z, A}\right\rangle\right|(\mathrm{V} 2, \mathrm{AF})$ \\
\hline PBE & 1.467 & 1.467 & 1.467 & 1.467 \\
\hline BP86 & 1.465 & 1.465 & 1.465 & 1.465 \\
\hline TPSSH & 1.498 & 1.498 & 1.498 & 1.498 \\
\hline B3LYP & 1.477 & 1.477 & 1.477 & 1.477 \\
\hline LC- $\omega$ PBE & 1.536 & 1.536 & 1.536 & 1.536 \\
\hline \multicolumn{5}{|c|}{ BS structure } \\
\hline $\mathrm{PBE}$ & 1.467 & 1.467 & 1.467 & 1.467 \\
\hline BP86 & 1.465 & 1.465 & 1.465 & 1.465 \\
\hline TPSSH & 1.497 & 1.497 & 1.497 & 1.497 \\
\hline B3LYP & 1.477 & 1.477 & 1.477 & 1.477 \\
\hline $\mathrm{LC}-\omega \mathrm{PBE}$ & 1.536 & 1.536 & 1.536 & 1.536 \\
\hline
\end{tabular}


Table S4: Mulliken local spins for the both spin-state structures and both spin states of calculated with different exchange-correlation functionals for the $\left[\mathrm{V}_{2}(\mu-\mathrm{O}-\mathrm{Me})_{2}(\mathrm{O})_{2}\left(\mathrm{ma}_{2}\right)\right]$ complex. The values are given in a.u.. Basis set: defTZVP.

\begin{tabular}{|c|cc|cc|}
\hline \hline \multicolumn{5}{|c|}{ high-spin structure } \\
\hline Functional & $\left|\left\langle\hat{S}_{z, A}\right\rangle\right|(\mathrm{V} 1, \mathrm{~F})$ & $\left|\left\langle\hat{S}_{z, A}\right\rangle\right|(\mathrm{V} 2, \mathrm{~F})$ & $\left|\left\langle\hat{S}_{z, A}\right\rangle\right|(\mathrm{V} 1, \mathrm{AF})$ & $\left|\left\langle\hat{S}_{z, A}\right\rangle\right|(\mathrm{V} 2, \mathrm{AF})$ \\
\hline PBE & 0.577 & 0.577 & 0.565 & 0.566 \\
BP86 & 0.575 & 0.575 & 0.562 & 0.563 \\
TPSSH & 0.591 & 0.591 & 0.586 & 0.587 \\
B3LYP & 0.588 & 0.588 & 0.584 & 0.585 \\
LC- $\omega$ PBE & 0.605 & 0.605 & 0.562 & 0.563 \\
\hline & & BS structure & 0.556 \\
PBE & 0.575 & 0.575 & 0.555 & 0.553 \\
BP86 & 0.572 & 0.572 & 0.552 & 0.582 \\
TPSSH & 0.589 & 0.589 & 0.581 & 0.5813 \\
B3LYP & 0.585 & 0.585 & 0.581 & 0.613 \\
LC- $\omega$ PBE & 0.603 & 0.603 & & \\
\hline \hline
\end{tabular}




\section{Larger systems with delocalized spins}

Table S5: Magnetic coupling constants calculated with the Broken-Symmetry approach from the optimized spin-state structures of $\left[\mathrm{Co}_{2}\left(\eta^{5}-\mathrm{Cp}\right)_{2}\left(\mu-\eta^{5}-1,8-\mathrm{DCN}\right)\right]$. All values are given in $\mathrm{kJ} / \mathrm{mol}$. Basis set: def-TZVP.

\begin{tabular}{c|cc|}
\hline \hline Functional & $J_{\mathrm{BS}, \text { unprojected }}$ & $J_{\mathrm{BS}, \text { projected }}$ \\
\hline PBE & 0.69 & 1.37 \\
BP86 & 0.72 & 1.43 \\
TPSSH & -0.22 & -0.43 \\
B3LYP & 0.47 & 0.94 \\
LC- $\omega$ PBE & 23.02 & 46.04 \\
\hline \hline
\end{tabular}




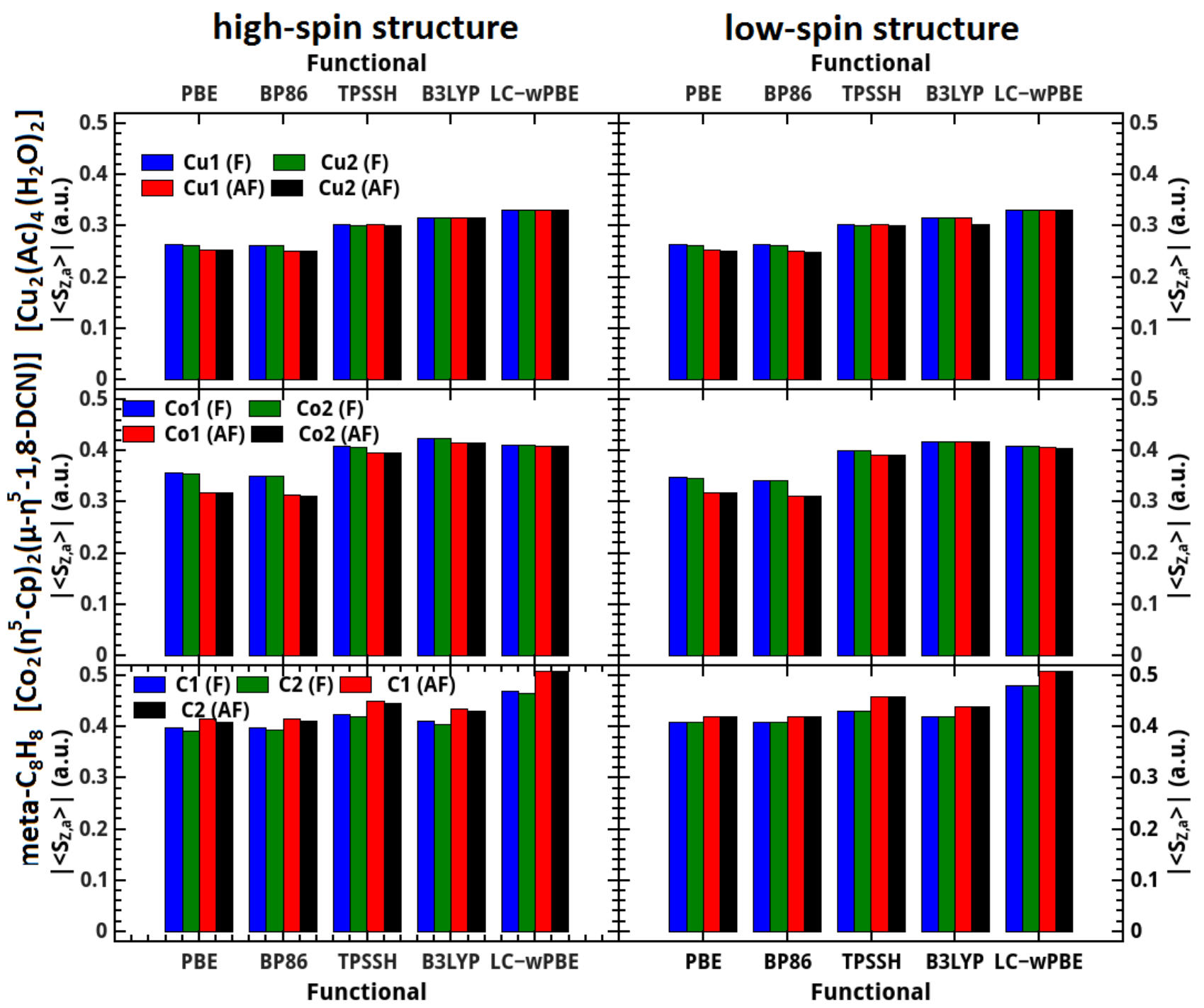

Figure S4: Mulliken local spins of all structures with delocalized spins for the high-spin structure (left column) and the BS structure (right column). 


\subsection{Local spins on defined magnetic centers}

Table S6: Mulliken local spins for the both spin-state structures and both spin states of the $\left[\mathrm{Cu}_{2}(\mathrm{Ac})_{4}\left(\mathrm{H}_{2} \mathrm{O}\right)_{2}\right]$ complex calculated with different exchange-correlation functionals. The values are given in a.u.. Basis set: def-TZVP.

\begin{tabular}{|c|cc|cc|}
\hline \hline \multicolumn{5}{|c|}{ high-spin structure } \\
\hline Functional & $\left|\left\langle\hat{S}_{z, A}\right\rangle\right|(\mathrm{Cu} 1, \mathrm{~F})$ & $\left|\left\langle\hat{S}_{z, A}\right\rangle\right|(\mathrm{Cu} 2, \mathrm{~F})$ & $\left|\left\langle\hat{S}_{z, A}\right\rangle\right|(\mathrm{Cu} 1, \mathrm{AF})$ & $\left|\left\langle\hat{S}_{z, A}\right\rangle\right|(\mathrm{Cu} 2, \mathrm{AF})$ \\
\hline PBE & 0.264 & 0.263 & 0.254 & 0.252 \\
BP86 & 0.263 & 0.261 & 0.252 & 0.250 \\
TPSSH & 0.303 & 0.302 & 0.303 & 0.302 \\
B3LYP & 0.317 & 0.316 & 0.303 & 0.316 \\
LC- $\omega$ PBE & 0.332 & 0.330 & 0.332 & 0.331 \\
\hline & & BS structure & & 0.252 \\
PBE & 0.264 & 0.263 & 0.253 & 0.250 \\
BP86 & 0.263 & 0.262 & 0.251 & 0.302 \\
TPSSH & 0.303 & 0.302 & 0.303 & 0.331 \\
B3LYP & 0.317 & 0.316 & 0.317 & 0.332 \\
LC- $\omega$ PBE & 0.332 & 0.331 & & \\
\hline \hline
\end{tabular}


Table S7: Mulliken local spins for the both spin-state structures and both spin states of the $\left[\mathrm{Co}_{2}\left(\eta^{5}-\mathrm{Cp}\right)_{2}\left(\mu-\eta^{5}-1,8-\mathrm{DCN}\right)\right]$ complex calculated with different exchangecorrelation functionals. The values are given in a.u.. Basis set: def-TZVP.

\begin{tabular}{|c|cc|cc|}
\hline \hline \multicolumn{5}{|c|}{ high-spin structure } \\
\hline Functional & $\left|\left\langle\hat{S}_{z, A}\right\rangle\right|(\mathrm{Co} 1, \mathrm{~F})$ & $\left|\left\langle\hat{S}_{z, A}\right\rangle\right|(\mathrm{Co} 2, \mathrm{~F})$ & $\left|\left\langle\hat{S}_{z, A}\right\rangle\right|(\mathrm{Co} 1, \mathrm{AF})$ & $\left|\left\langle\hat{S}_{z, A}\right\rangle\right|(\mathrm{Co} 2, \mathrm{AF})$ \\
\hline PBE & 0.357 & 0.356 & 0.319 & 0.319 \\
BP86 & 0.352 & 0.351 & 0.313 & 0.313 \\
TPSSH & 0.409 & 0.408 & 0.396 & 0.396 \\
B3LYP & 0.425 & 0.424 & 0.416 & 0.416 \\
LC- $\omega$ PBE & 0.413 & 0.412 & 0.410 & 0.410 \\
\hline & & BS structure & & 0.318 \\
PBE & 0.348 & 0.348 & 0.319 & 0.312 \\
BP86 & 0.343 & 0.342 & 0.313 & 0.392 \\
TPSSH & 0.402 & 0.401 & 0.393 & 0.419 \\
B3LYP & 0.419 & 0.419 & 0.419 & 0.408 \\
LC- $\omega$ PBE & 0.410 & 0.409 & & \\
\hline \hline
\end{tabular}




\subsection{Mulliken local spin densities on all atoms for the high-spin structure of the $\left[\mathrm{Cu}_{2}(\mathrm{Ac})_{4}\left(\mathrm{H}_{2} \mathrm{O}\right)_{2}\right]$ complex}

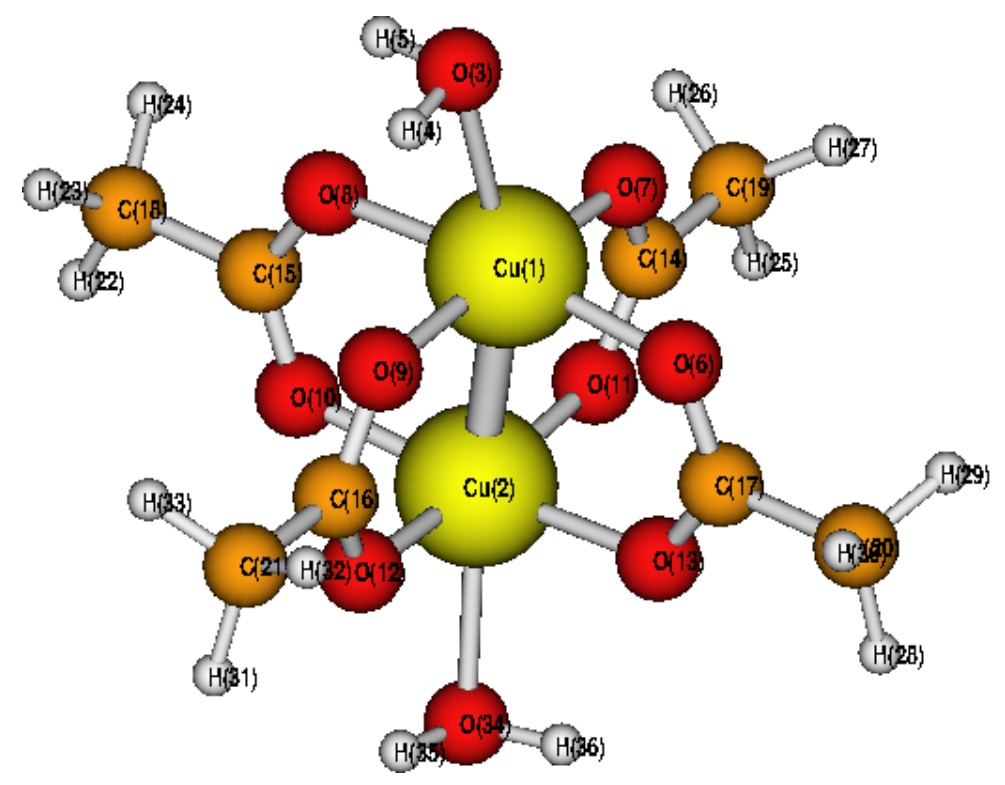

Figure S5: High-spin structure of the $\left[\mathrm{Cu}_{2}(\mathrm{Ac})_{4}\left(\mathrm{H}_{2} \mathrm{O}\right)_{2}\right]$ complex and the corresponding atomic numbers as used for the atomic spin densities below. The same numbering scheme was used for the Broken-Symmetry solution.

Table S8: Mulliken local spin densities for both spin states of $\left[\mathrm{Cu}_{2}(\mathrm{Ac})_{4}\left(\mathrm{H}_{2} \mathrm{O}\right)_{2}\right]$ calculated with PBE. The values are given in a.u.. Basis set: def-TZVP.

\begin{tabular}{|c|c|c|c|}
\hline \hline Number & Element & Spin density (high-spin) & Spin density (BS solution) \\
\hline 1 & $\mathrm{Cu}$ & 0.527509 & 0.507253 \\
2 & $\mathrm{Cu}$ & 0.524753 & -0.504690 \\
3 & $\mathrm{O}$ & -0.000253 & -0.000275 \\
4 & $\mathrm{H}$ & -0.000184 & -0.000115 \\
\hline
\end{tabular}




\begin{tabular}{|c|c|c|c|}
\hline 5 & $\mathrm{H}$ & -0.000163 & -0.000077 \\
\hline 6 & $\mathrm{O}$ & 0.113839 & 0.103863 \\
\hline 7 & $\mathrm{O}$ & 0.122462 & 0.111234 \\
\hline 8 & $\mathrm{O}$ & 0.102472 & 0.093266 \\
\hline 9 & $\mathrm{O}$ & 0.097264 & 0.088763 \\
\hline 10 & $\mathrm{O}$ & 0.114507 & -0.104185 \\
\hline 11 & $\mathrm{O}$ & 0.122770 & -0.111149 \\
\hline 12 & $\mathrm{O}$ & 0.096606 & -0.087872 \\
\hline 13 & $\mathrm{O}$ & 0.104739 & -0.094860 \\
\hline 14 & $\mathrm{C}$ & -0.006272 & -0.000254 \\
\hline 15 & $\mathrm{C}$ & -0.005097 & 0.000202 \\
\hline 16 & $\mathrm{C}$ & -0.004242 & -0.000412 \\
\hline 17 & $\mathrm{C}$ & -0.005195 & -0.000852 \\
\hline 18 & $\mathrm{C}$ & 0.018426 & -0.001481 \\
\hline 19 & $\mathrm{C}$ & 0.020823 & -0.000746 \\
\hline 20 & $\mathrm{C}$ & 0.018435 & 0.000224 \\
\hline 21 & $\mathrm{C}$ & 0.016261 & -0.000472 \\
\hline 22 & $\mathrm{H}$ & 0.003524 & 0.002961 \\
\hline 23 & $\mathrm{H}$ & 0.000475 & -0.001018 \\
\hline
\end{tabular}




\begin{tabular}{|c|c|c|c|}
\hline 24 & $\mathrm{H}$ & 0.001216 & -0.001630 \\
\hline 25 & $\mathrm{H}$ & 0.003999 & 0.003418 \\
\hline 26 & $\mathrm{H}$ & 0.000346 & -0.001086 \\
\hline 27 & $\mathrm{H}$ & 0.001360 & -0.001767 \\
\hline 28 & $\mathrm{H}$ & 0.003886 & 0.003271 \\
\hline 29 & $\mathrm{H}$ & 0.000577 & -0.001200 \\
\hline 30 & $\mathrm{H}$ & 0.000869 & -0.001334 \\
\hline 31 & $\mathrm{H}$ & 0.003461 & 0.002874 \\
\hline 32 & $\mathrm{H}$ & 0.000617 & -0.001092 \\
\hline 33 & $\mathrm{H}$ & 0.000826 & -0.001261 \\
\hline 34 & $\mathrm{O}$ & -0.000237 & 0.000253 \\
\hline 35 & $\mathrm{H}$ & -0.000183 & 0.000119 \\
\hline 36 & $\mathrm{H}$ & -0.000197 & 0.000128 \\
\hline
\end{tabular}

Table S9: Mulliken local spin densities for both spin states of $\left[\mathrm{Cu}_{2}(\mathrm{Ac})_{4}\left(\mathrm{H}_{2} \mathrm{O}\right)_{2}\right]$ calculated with BP86. The values are given in a.u.. Basis set: def-TZVP.

\begin{tabular}{|c|c|c|c|}
\hline \hline Number & Element & Spin density (high-spin) & Spin density (BS solution) \\
\hline 1 & $\mathrm{Cu}$ & 0.525221 & 0.503231 \\
2 & $\mathrm{Cu}$ & 0.522459 & -0.500644 \\
\hline
\end{tabular}




\begin{tabular}{|c|c|c|c|}
\hline 3 & $\mathrm{O}$ & -0.000213 & -0.000236 \\
\hline 4 & $\mathrm{H}$ & -0.000171 & -0.000109 \\
\hline 5 & $\mathrm{H}$ & -0.000153 & -0.000076 \\
\hline 6 & $\mathrm{O}$ & 0.114387 & 0.103729 \\
\hline 7 & $\mathrm{O}$ & 0.123130 & 0.111130 \\
\hline 8 & $\mathrm{O}$ & 0.102969 & 0.093095 \\
\hline 9 & $\mathrm{O}$ & 0.097654 & 0.088570 \\
\hline 10 & $\mathrm{O}$ & 0.115037 & -0.104074 \\
\hline 11 & $\mathrm{O}$ & 0.123435 & -0.111083 \\
\hline 12 & $\mathrm{O}$ & 0.096987 & -0.087701 \\
\hline 13 & $\mathrm{O}$ & 0.105215 & -0.094707 \\
\hline 14 & $\mathrm{C}$ & -0.006534 & -0.000194 \\
\hline 15 & $\mathrm{C}$ & -0.005308 & 0.000269 \\
\hline 16 & $\mathrm{C}$ & -0.004384 & -0.000361 \\
\hline 17 & $\mathrm{C}$ & -0.005395 & -0.000796 \\
\hline 18 & $\mathrm{C}$ & 0.019060 & -0.001537 \\
\hline 19 & $\mathrm{C}$ & 0.021483 & -0.000802 \\
\hline 20 & $\mathrm{C}$ & 0.019055 & 0.000185 \\
\hline 21 & $\mathrm{C}$ & 0.016858 & -0.000512 \\
\hline
\end{tabular}




\begin{tabular}{|c|c|c|c|}
\hline 22 & $\mathrm{H}$ & 0.003373 & 0.002959 \\
\hline 23 & $\mathrm{H}$ & 0.000383 & -0.001012 \\
\hline 24 & $\mathrm{H}$ & 0.001106 & -0.001615 \\
\hline 25 & $\mathrm{H}$ & 0.003851 & 0.003417 \\
\hline 26 & $\mathrm{H}$ & 0.000255 & -0.001078 \\
\hline 27 & $\mathrm{H}$ & 0.001247 & -0.001757 \\
\hline 28 & $\mathrm{H}$ & 0.003743 & 0.003258 \\
\hline 29 & $\mathrm{H}$ & 0.000479 & -0.001191 \\
\hline 30 & $\mathrm{H}$ & 0.000763 & -0.001326 \\
\hline 31 & $\mathrm{H}$ & 0.003315 & 0.002858 \\
\hline 32 & $\mathrm{H}$ & 0.000519 & -0.001085 \\
\hline 33 & $\mathrm{H}$ & 0.000724 & -0.001249 \\
\hline 34 & $\mathrm{O}$ & -0.000198 & 0.000216 \\
\hline 35 & $\mathrm{H}$ & -0.000170 & 0.000112 \\
\hline 36 & $\mathrm{H}$ & -0.000182 & 0.000119 \\
\hline
\end{tabular}

Table S10: Mulliken local spin densities for both spin states of $\left[\mathrm{Cu}_{2}(\mathrm{Ac})_{4}\left(\mathrm{H}_{2} \mathrm{O}\right)_{2}\right]$ calculated with TPSSH. The values are given in a.u.. Basis set: def-TZVP.

\begin{tabular}{|l|l|l|l|}
\hline \hline Number & Element & Spin density (high-spin) & Spin density (BS solution) \\
\hline
\end{tabular}




\begin{tabular}{|c|c|c|c|}
\hline 1 & $\mathrm{Cu}$ & 0.605882 & 0.605711 \\
\hline 2 & $\mathrm{Cu}$ & 0.603376 & -0.603294 \\
\hline 3 & $\mathrm{O}$ & -0.000156 & -0.000141 \\
\hline 4 & $\mathrm{H}$ & -0.000170 & -0.000121 \\
\hline 5 & $\mathrm{H}$ & -0.000166 & -0.000097 \\
\hline 6 & $\mathrm{O}$ & 0.097205 & 0.095417 \\
\hline 7 & $\mathrm{O}$ & 0.104420 & 0.102061 \\
\hline 8 & $\mathrm{O}$ & 0.086883 & 0.085165 \\
\hline 9 & $\mathrm{O}$ & 0.082594 & 0.081161 \\
\hline 10 & $\mathrm{O}$ & 0.097847 & -0.095854 \\
\hline 11 & $\mathrm{O}$ & 0.104698 & -0.102120 \\
\hline 12 & $\mathrm{O}$ & 0.081908 & -0.080342 \\
\hline 13 & $\mathrm{O}$ & 0.088942 & -0.086865 \\
\hline 14 & $\mathrm{C}$ & -0.008897 & 0.000014 \\
\hline 15 & $\mathrm{C}$ & -0.007501 & 0.000688 \\
\hline 16 & $\mathrm{C}$ & -0.006378 & -0.000181 \\
\hline 17 & $\mathrm{C}$ & -0.007575 & -0.000808 \\
\hline 18 & $\mathrm{C}$ & 0.016040 & -0.001490 \\
\hline 19 & $\mathrm{C}$ & 0.018136 & -0.000840 \\
\hline
\end{tabular}




\begin{tabular}{|c|c|c|c|}
\hline 20 & $\mathrm{C}$ & 0.016040 & 0.000052 \\
\hline 21 & $\mathrm{C}$ & 0.014162 & -0.000561 \\
\hline 22 & $\mathrm{H}$ & 0.002410 & 0.002528 \\
\hline 23 & $\mathrm{H}$ & 0.000122 & -0.000826 \\
\hline 24 & $\mathrm{H}$ & 0.000713 & -0.001353 \\
\hline 25 & $\mathrm{H}$ & 0.002762 & 0.002916 \\
\hline 26 & $\mathrm{H}$ & 0.000025 & -0.000895 \\
\hline 27 & $\mathrm{H}$ & 0.000789 & -0.001468 \\
\hline 28 & $\mathrm{H}$ & 0.002703 & 0.002782 \\
\hline 29 & $\mathrm{H}$ & 0.000218 & -0.000992 \\
\hline 30 & $\mathrm{H}$ & 0.000421 & -0.001115 \\
\hline 31 & $\mathrm{H}$ & 0.002380 & 0.002439 \\
\hline 32 & $\mathrm{H}$ & 0.000242 & -0.000890 \\
\hline 33 & $\mathrm{H}$ & 0.000418 & -0.001054 \\
\hline 34 & $\mathrm{O}$ & -0.000139 & 0.000121 \\
\hline 35 & $\mathrm{H}$ & -0.000170 & 0.000123 \\
\hline 36 & $\mathrm{H}$ & -0.000184 & 0.000128 \\
\hline
\end{tabular}


Table S11: Mulliken local spin densities for both spin states of $\left[\mathrm{Cu}_{2}(\mathrm{Ac})_{4}\left(\mathrm{H}_{2} \mathrm{O}\right)_{2}\right]$ calculated with B3LYP. The values are given in a.u.. Basis set: def-TZVP.

\begin{tabular}{|c|c|c|c|}
\hline Number & Element & Spin density (high-spin) & Spin density (BS solution) \\
\hline 1 & $\mathrm{Cu}$ & 0.633247 & 0.633254 \\
\hline 2 & $\mathrm{Cu}$ & 0.631067 & -0.631096 \\
\hline 3 & $\mathrm{O}$ & -0.000242 & -0.000224 \\
\hline 4 & $\mathrm{H}$ & -0.000187 & -0.000141 \\
\hline 5 & $\mathrm{H}$ & -0.000183 & -0.000119 \\
\hline 6 & $\mathrm{O}$ & 0.091490 & 0.091966 \\
\hline 7 & $\mathrm{O}$ & 0.098059 & 0.098343 \\
\hline 8 & $\mathrm{O}$ & 0.081465 & 0.081953 \\
\hline 9 & $\mathrm{O}$ & 0.077649 & 0.078151 \\
\hline 10 & $\mathrm{O}$ & 0.092150 & -0.092449 \\
\hline 11 & $\mathrm{O}$ & 0.098322 & -0.098444 \\
\hline 12 & $\mathrm{O}$ & 0.076944 & -0.077351 \\
\hline 13 & $\mathrm{O}$ & 0.083473 & -0.083681 \\
\hline 14 & $\mathrm{C}$ & -0.008423 & 0.000048 \\
\hline 15 & $\mathrm{C}$ & -0.007130 & 0.000780 \\
\hline
\end{tabular}




\begin{tabular}{|c|c|c|c|}
\hline 16 & $\mathrm{C}$ & -0.006087 & -0.000132 \\
\hline 17 & $\mathrm{C}$ & -0.007212 & -0.000829 \\
\hline 18 & $\mathrm{C}$ & 0.013641 & -0.001418 \\
\hline 19 & $\mathrm{C}$ & 0.015466 & -0.000835 \\
\hline 20 & $\mathrm{C}$ & 0.013628 & -0.000044 \\
\hline 21 & $\mathrm{C}$ & 0.011992 & -0.000576 \\
\hline 22 & $\mathrm{H}$ & 0.002089 & 0.002091 \\
\hline 23 & $\mathrm{H}$ & 0.000109 & -0.000601 \\
\hline 24 & $\mathrm{H}$ & 0.000616 & -0.001049 \\
\hline 25 & $\mathrm{H}$ & 0.002388 & 0.002421 \\
\hline 26 & $\mathrm{H}$ & 0.000030 & -0.000665 \\
\hline 27 & $\mathrm{H}$ & 0.000686 & -0.001130 \\
\hline 28 & $\mathrm{H}$ & 0.002335 & 0.002316 \\
\hline 29 & $\mathrm{H}$ & 0.000200 & -0.000750 \\
\hline 30 & $\mathrm{H}$ & 0.000379 & -0.000840 \\
\hline 31 & $\mathrm{H}$ & 0.002062 & 0.002025 \\
\hline 32 & $\mathrm{H}$ & 0.000215 & -0.000662 \\
\hline 33 & $\mathrm{H}$ & 0.000373 & -0.000803 \\
\hline 34 & $\mathrm{O}$ & -0.000226 & 0.000208 \\
\hline
\end{tabular}




\begin{tabular}{|l|l|l|l|}
35 & $\mathrm{H}$ & -0.000185 & 0.000139 \\
36 & $\mathrm{H}$ & -0.000201 & 0.000146 \\
\hline
\end{tabular}

Table S12: Mulliken local spin densities for both spin states of $\left[\mathrm{Cu}_{2}(\mathrm{Ac})_{4}\left(\mathrm{H}_{2} \mathrm{O}\right)_{2}\right]$ calculated with LC- $\omega$ PBE. The values are given in a.u.. Basis set: def-TZVP.

\begin{tabular}{|c|c|c|c|}
\hline Number & Element & Spin density (high-spin) & Spin density (BS solution) \\
\hline 1 & $\mathrm{Cu}$ & 0.662536 & 0.663260 \\
\hline 2 & $\mathrm{Cu}$ & 0.660905 & -0.661668 \\
\hline 3 & $\mathrm{O}$ & -0.000087 & -0.000064 \\
\hline 4 & $\mathrm{H}$ & -0.000114 & -0.000084 \\
\hline 5 & $\mathrm{H}$ & -0.000113 & -0.000070 \\
\hline 6 & $\mathrm{O}$ & 0.085428 & 0.087052 \\
\hline 7 & $\mathrm{O}$ & 0.091730 & 0.093336 \\
\hline 8 & $\mathrm{O}$ & 0.075033 & 0.076577 \\
\hline 9 & $\mathrm{O}$ & 0.071568 & 0.073028 \\
\hline 10 & $\mathrm{O}$ & 0.086072 & -0.087568 \\
\hline 11 & $\mathrm{O}$ & 0.091858 & -0.093381 \\
\hline 12 & $\mathrm{O}$ & 0.070759 & -0.072168 \\
\hline 13 & $\mathrm{O}$ & 0.077023 & -0.078395 \\
\hline
\end{tabular}




\begin{tabular}{|c|c|c|c|}
\hline 14 & $\mathrm{C}$ & -0.008128 & 0.000129 \\
\hline 15 & $\mathrm{C}$ & -0.006732 & 0.000946 \\
\hline 16 & $\mathrm{C}$ & -0.005563 & -0.000052 \\
\hline 17 & $\mathrm{C}$ & -0.006772 & -0.000805 \\
\hline 18 & $\mathrm{C}$ & 0.012179 & -0.001308 \\
\hline 19 & $\mathrm{C}$ & 0.013800 & -0.000733 \\
\hline 20 & $\mathrm{C}$ & 0.012160 & -0.000034 \\
\hline 21 & $\mathrm{C}$ & 0.010719 & -0.000514 \\
\hline 22 & $\mathrm{H}$ & 0.001460 & 0.001604 \\
\hline 23 & $\mathrm{H}$ & -0.000212 & -0.000383 \\
\hline 24 & $\mathrm{H}$ & 0.000216 & -0.000744 \\
\hline 25 & $\mathrm{H}$ & 0.001682 & 0.001861 \\
\hline 26 & $\mathrm{H}$ & -0.000302 & -0.000454 \\
\hline 27 & $\mathrm{H}$ & 0.000247 & -0.000794 \\
\hline 28 & $\mathrm{H}$ & 0.001660 & 0.001788 \\
\hline 29 & $\mathrm{H}$ & -0.000132 & -0.000538 \\
\hline 30 & $\mathrm{H}$ & 0.000027 & -0.000581 \\
\hline 31 & $\mathrm{H}$ & 0.001456 & 0.001559 \\
\hline 32 & $\mathrm{H}$ & -0.000097 & -0.000451 \\
\hline
\end{tabular}




\begin{tabular}{|l|l|l|l|}
33 & $\mathrm{H}$ & 0.000043 & -0.000566 \\
34 & $\mathrm{O}$ & -0.000072 & 0.000049 \\
35 & $\mathrm{H}$ & -0.000113 & 0.000082 \\
36 & $\mathrm{H}$ & -0.000125 & 0.000088 \\
\hline
\end{tabular}

\subsection{Mulliken local spin densities on all atoms for the high- spin structure of the $\left[\mathbf{C o}_{2}\left(\eta^{5}-\mathbf{C p}\right)_{2}\left(\mu-\eta^{5}-1,8-\mathbf{D C N}\right)\right]$ complex}
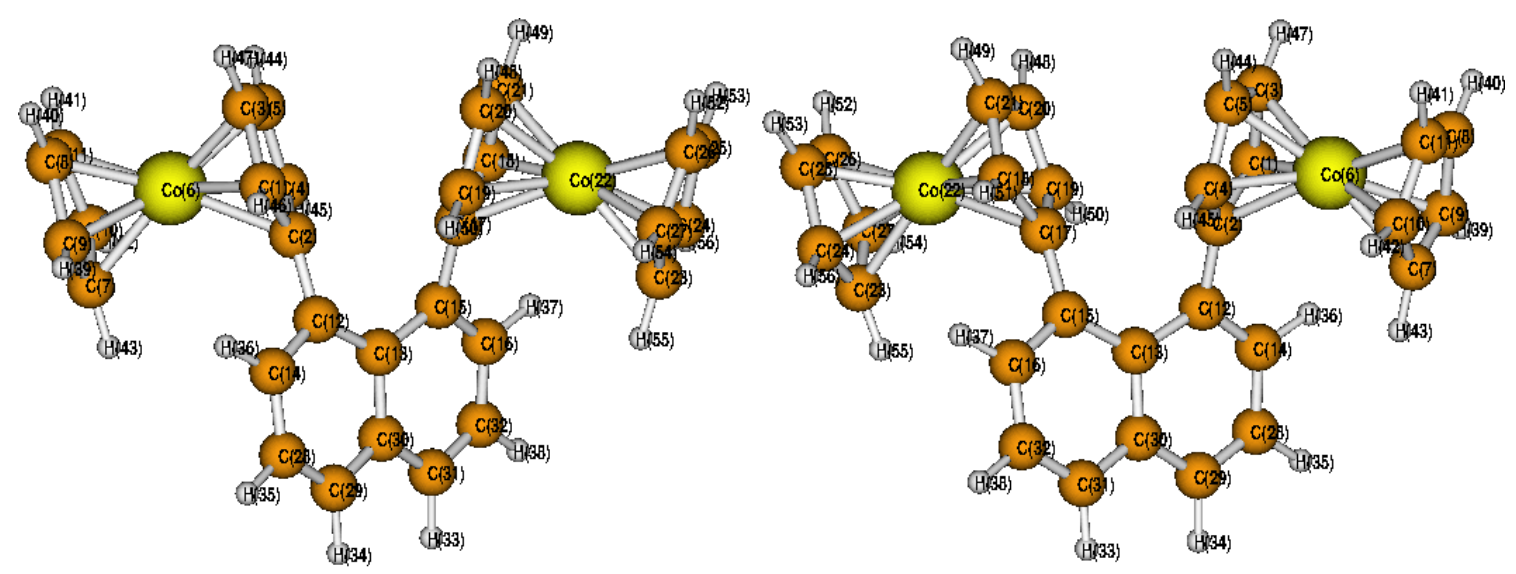

Figure S6: High-spin structure of the $\left[\mathrm{Co}_{2}\left(\eta^{5}-\mathrm{Cp}\right)_{2}\left(\mu-\eta^{5}-1,8-\mathrm{DCN}\right)\right]$ complex (in two orientations) and the corresponding atomic numbers as used for the atomic spin densities below. The same numbering scheme was used for the Broken-Symmetry solution. 
Table S13: Mulliken local spin densities for both spin states of $\left[\mathrm{Co}_{2}\left(\eta^{5}-\mathrm{Cp}\right)_{2}\left(\mu-\eta^{5}-1,8-\mathrm{DCN}\right)\right]$ calculated with PBE. The values are given in a.u.. Basis set: def-TZVP.

\begin{tabular}{|c|c|c|c|}
\hline Number & Element & Spin density (high-spin) & Spin density (BS solution) \\
\hline 1 & $\mathrm{C}$ & -0.015780 & -0.027020 \\
\hline 2 & $\mathrm{C}$ & 0.105034 & 0.107285 \\
\hline 3 & $\mathrm{C}$ & -0.008823 & 0.000085 \\
\hline 4 & $\mathrm{C}$ & -0.043612 & -0.035159 \\
\hline 5 & $\mathrm{C}$ & 0.047735 & 0.050009 \\
\hline 6 & $\mathrm{Co}$ & 0.713713 & 0.637997 \\
\hline 7 & $\mathrm{C}$ & 0.065428 & 0.073797 \\
\hline 8 & $\mathrm{C}$ & -0.002006 & -0.002810 \\
\hline 9 & $\mathrm{C}$ & 0.016056 & 0.003106 \\
\hline 10 & $\mathrm{C}$ & -0.026318 & -0.040074 \\
\hline 11 & $\mathrm{C}$ & 0.082329 & 0.085524 \\
\hline 12 & $\mathrm{C}$ & -0.013411 & 0.002037 \\
\hline 13 & $\mathrm{C}$ & 0.011052 & -0.000080 \\
\hline 14 & $\mathrm{C}$ & 0.065473 & 0.032496 \\
\hline 15 & $\mathrm{C}$ & -0.013834 & -0.001769 \\
\hline
\end{tabular}




\begin{tabular}{|c|c|c|c|}
\hline 16 & $\mathrm{C}$ & 0.066425 & -0.034324 \\
\hline 17 & $\mathrm{C}$ & 0.105602 & -0.107360 \\
\hline 18 & $\mathrm{C}$ & -0.016231 & 0.027319 \\
\hline 19 & $\mathrm{C}$ & -0.042876 & 0.035080 \\
\hline 20 & $\mathrm{C}$ & 0.046647 & -0.049218 \\
\hline 21 & $\mathrm{C}$ & -0.008732 & 0.000241 \\
\hline 22 & Co & 0.711042 & -0.636980 \\
\hline 23 & $\mathrm{C}$ & 0.055131 & -0.061973 \\
\hline 24 & $\mathrm{C}$ & 0.028295 & -0.017594 \\
\hline 25 & $\mathrm{C}$ & -0.010173 & 0.013277 \\
\hline 26 & $\mathrm{C}$ & 0.084558 & -0.089820 \\
\hline 27 & $\mathrm{C}$ & -0.022675 & 0.036831 \\
\hline 28 & $\mathrm{C}$ & -0.021153 & -0.006356 \\
\hline 29 & $\mathrm{C}$ & 0.059140 & 0.032502 \\
\hline 30 & $\mathrm{C}$ & -0.027806 & 0.000425 \\
\hline 31 & $\mathrm{C}$ & 0.060272 & -0.034334 \\
\hline 32 & $\mathrm{C}$ & -0.021374 & 0.006937 \\
\hline 33 & $\mathrm{H}$ & -0.003266 & 0.002532 \\
\hline 34 & $\mathrm{H}$ & -0.003192 & -0.002422 \\
\hline
\end{tabular}




\begin{tabular}{|c|c|c|c|}
\hline 35 & $\mathrm{H}$ & 0.000752 & 0.000221 \\
\hline 36 & $\mathrm{H}$ & -0.003176 & -0.001433 \\
\hline 37 & $\mathrm{H}$ & -0.003226 & 0.001530 \\
\hline 38 & $\mathrm{H}$ & 0.000748 & -0.000234 \\
\hline 39 & $\mathrm{H}$ & -0.001030 & -0.000419 \\
\hline 40 & $\mathrm{H}$ & -0.000251 & -0.000152 \\
\hline 41 & $\mathrm{H}$ & -0.004333 & -0.004382 \\
\hline 42 & $\mathrm{H}$ & 0.000640 & 0.001060 \\
\hline 43 & $\mathrm{H}$ & -0.003749 & -0.004071 \\
\hline 44 & $\mathrm{H}$ & -0.001825 & -0.001885 \\
\hline 45 & $\mathrm{H}$ & -0.000082 & 0.002017 \\
\hline 46 & $\mathrm{H}$ & 0.001272 & 0.000726 \\
\hline 47 & $\mathrm{H}$ & 0.000514 & -0.000311 \\
\hline 48 & $\mathrm{H}$ & -0.001782 & 0.001840 \\
\hline 49 & $\mathrm{H}$ & 0.000499 & 0.000301 \\
\hline 50 & $\mathrm{H}$ & -0.000203 & -0.002097 \\
\hline 51 & $\mathrm{H}$ & 0.001206 & -0.000753 \\
\hline 52 & $\mathrm{H}$ & -0.004432 & 0.004580 \\
\hline 53 & $\mathrm{H}$ & 0.000022 & -0.000196 \\
\hline
\end{tabular}




\begin{tabular}{|l|l|l|l|}
54 & $\mathrm{H}$ & 0.000513 & -0.000977 \\
55 & $\mathrm{H}$ & -0.003233 & 0.003450 \\
56 & $\mathrm{H}$ & -0.001515 & 0.000994 \\
\hline
\end{tabular}

Table S14: Mulliken local spin densities for both spin states of $\left[\mathrm{Co}_{2}\left(\eta^{5}-\mathrm{Cp}\right)_{2}\left(\mu-\eta^{5}-1,8-\mathrm{DCN}\right)\right]$ calculated with BP86. The values are given in a.u.. Basis set: def-TZVP.

\begin{tabular}{|c|c|c|c|}
\hline Number & Element & Spin density (high-spin) & Spin density (BS solution) \\
\hline 1 & $\mathrm{C}$ & -0.014688 & -0.040394 \\
\hline 2 & $\mathrm{C}$ & 0.107438 & 0.123868 \\
\hline 3 & $\mathrm{C}$ & -0.008652 & 0.020443 \\
\hline 4 & $\mathrm{C}$ & -0.043058 & -0.036818 \\
\hline 5 & $\mathrm{C}$ & 0.050585 & 0.034828 \\
\hline 6 & $\mathrm{Co}$ & 0.703382 & 0.624880 \\
\hline 7 & $\mathrm{C}$ & 0.067649 & 0.091618 \\
\hline 8 & $\mathrm{C}$ & -0.001954 & 0.023674 \\
\hline 9 & $\mathrm{C}$ & 0.017236 & -0.016963 \\
\hline 10 & $\mathrm{C}$ & -0.026871 & -0.037894 \\
\hline 11 & $\mathrm{C}$ & 0.085573 & 0.065185 \\
\hline
\end{tabular}




\begin{tabular}{|c|c|c|c|}
\hline 12 & $\mathrm{C}$ & -0.016600 & -0.001336 \\
\hline 13 & $\mathrm{C}$ & 0.013008 & -0.000333 \\
\hline 14 & $\mathrm{C}$ & 0.069675 & 0.039229 \\
\hline 15 & $\mathrm{C}$ & -0.017256 & 0.001832 \\
\hline 16 & $\mathrm{C}$ & 0.070772 & -0.040945 \\
\hline 17 & $\mathrm{C}$ & 0.108378 & -0.124327 \\
\hline 18 & $\mathrm{C}$ & -0.015180 & 0.040880 \\
\hline 19 & $\mathrm{C}$ & -0.042421 & 0.036795 \\
\hline 20 & $\mathrm{C}$ & 0.049521 & -0.034389 \\
\hline 21 & $\mathrm{C}$ & -0.008640 & -0.020102 \\
\hline 22 & $\mathrm{Co}$ & 0.700710 & -0.623457 \\
\hline 23 & $\mathrm{C}$ & 0.056984 & -0.084629 \\
\hline 24 & $\mathrm{C}$ & 0.029832 & 0.006381 \\
\hline 25 & $\mathrm{C}$ & -0.010389 & -0.011733 \\
\hline 26 & $\mathrm{C}$ & 0.087889 & -0.074649 \\
\hline 27 & $\mathrm{C}$ & -0.023089 & 0.039112 \\
\hline 28 & $\mathrm{C}$ & -0.023577 & -0.008927 \\
\hline 29 & $\mathrm{C}$ & 0.061348 & 0.038856 \\
\hline 30 & $\mathrm{C}$ & -0.030386 & 0.000429 \\
\hline
\end{tabular}




\begin{tabular}{|c|c|c|c|}
\hline 31 & $\mathrm{C}$ & 0.062526 & -0.040674 \\
\hline 32 & $\mathrm{C}$ & -0.023880 & 0.009551 \\
\hline 33 & $\mathrm{H}$ & -0.003446 & 0.003106 \\
\hline 34 & $\mathrm{H}$ & -0.003366 & -0.002996 \\
\hline 35 & $\mathrm{H}$ & 0.000767 & 0.000260 \\
\hline 36 & $\mathrm{H}$ & -0.003696 & -0.001880 \\
\hline 37 & $\mathrm{H}$ & -0.003775 & 0.001995 \\
\hline 38 & $\mathrm{H}$ & 0.000765 & -0.000274 \\
\hline 39 & $\mathrm{H}$ & -0.001279 & 0.000291 \\
\hline 40 & $\mathrm{H}$ & -0.000359 & -0.001475 \\
\hline 41 & $\mathrm{H}$ & -0.004844 & -0.003570 \\
\hline 42 & $\mathrm{H}$ & 0.000645 & 0.001022 \\
\hline 43 & $\mathrm{H}$ & -0.004426 & -0.005585 \\
\hline 44 & $\mathrm{H}$ & -0.002191 & -0.001571 \\
\hline 45 & $\mathrm{H}$ & -0.000568 & 0.002279 \\
\hline 46 & $\mathrm{H}$ & 0.000982 & 0.001155 \\
\hline 47 & $\mathrm{H}$ & 0.000485 & -0.001024 \\
\hline 48 & $\mathrm{H}$ & -0.002143 & 0.001562 \\
\hline 49 & $\mathrm{H}$ & 0.000473 & 0.000997 \\
\hline
\end{tabular}




\begin{tabular}{|l|l|l|l|}
50 & $\mathrm{H}$ & -0.000661 & -0.002320 \\
51 & $\mathrm{H}$ & 0.000935 & -0.001187 \\
52 & $\mathrm{H}$ & -0.004943 & 0.004075 \\
53 & $\mathrm{H}$ & -0.000036 & 0.000935 \\
54 & $\mathrm{H}$ & 0.000498 & -0.001076 \\
55 & $\mathrm{H}$ & -0.003841 & 0.005162 \\
56 & $\mathrm{H}$ & -0.001840 & 0.000127 \\
\hline \hline
\end{tabular}

Table S15: Mulliken local spin densities for both spin states of $\left[\mathrm{Co}_{2}\left(\eta^{5}-\mathrm{Cp}\right)_{2}\left(\mu-\eta^{5}-1,8-\mathrm{DCN}\right)\right]$ calculated with TPSSH. The values are given in a.u.. Basis set: def-TZVP.

\begin{tabular}{|c|c|c|c|}
\hline \hline Number & Element & Spin density (high-spin) & Spin density (BS solution) \\
\hline 1 & $\mathrm{C}$ & -0.044061 & -0.135650 \\
2 & $\mathrm{C}$ & 0.123084 & -0.109417 \\
3 & $\mathrm{C}$ & -0.009816 & -0.031860 \\
4 & $\mathrm{C}$ & -0.072227 & 0.069612 \\
5 & $\mathrm{C}$ & 0.052844 & -0.128034 \\
6 & $\mathrm{Co}$ & 0.816538 & -0.223034 \\
7 & $\mathrm{C}$ & 0.071030 & -0.115082 \\
\hline
\end{tabular}




\begin{tabular}{|c|c|c|c|}
\hline 8 & $\mathrm{C}$ & -0.018194 & -0.066441 \\
\hline 9 & $\mathrm{C}$ & 0.000675 & -0.101946 \\
\hline 10 & $\mathrm{C}$ & -0.053933 & -0.051327 \\
\hline 11 & $\mathrm{C}$ & 0.091854 & -0.129990 \\
\hline 12 & $\mathrm{C}$ & -0.031655 & 0.123413 \\
\hline 13 & $\mathrm{C}$ & 0.028404 & 0.184532 \\
\hline 14 & $\mathrm{C}$ & 0.063287 & -0.166288 \\
\hline 15 & $\mathrm{C}$ & -0.032582 & 0.129842 \\
\hline 16 & $\mathrm{C}$ & 0.064361 & -0.163645 \\
\hline 17 & $\mathrm{C}$ & 0.124365 & -0.100330 \\
\hline 18 & $\mathrm{C}$ & -0.045006 & -0.135925 \\
\hline 19 & $\mathrm{C}$ & -0.071621 & 0.066496 \\
\hline 20 & $\mathrm{C}$ & 0.051621 & -0.127287 \\
\hline 21 & $\mathrm{C}$ & -0.009364 & -0.033756 \\
\hline 22 & Co & 0.814963 & -0.222645 \\
\hline 23 & $\mathrm{C}$ & 0.057786 & -0.108105 \\
\hline 24 & $\mathrm{C}$ & 0.017216 & -0.111382 \\
\hline 25 & $\mathrm{C}$ & -0.030407 & -0.060096 \\
\hline 26 & $\mathrm{C}$ & 0.097669 & -0.134286 \\
\hline
\end{tabular}




\begin{tabular}{|c|c|c|c|}
\hline 27 & $\mathrm{C}$ & -0.050829 & -0.049847 \\
\hline 28 & $\mathrm{C}$ & -0.031176 & -0.056577 \\
\hline 29 & $\mathrm{C}$ & 0.055542 & -0.199004 \\
\hline 30 & $\mathrm{C}$ & -0.035629 & 0.023633 \\
\hline 31 & $\mathrm{C}$ & 0.056685 & -0.200238 \\
\hline 32 & $\mathrm{C}$ & -0.031557 & -0.059636 \\
\hline 33 & $\mathrm{H}$ & -0.003270 & 0.075922 \\
\hline 34 & $\mathrm{H}$ & -0.003191 & 0.076127 \\
\hline 35 & $\mathrm{H}$ & 0.001380 & 0.070188 \\
\hline 36 & $\mathrm{H}$ & -0.003220 & 0.079081 \\
\hline 37 & $\mathrm{H}$ & -0.003275 & 0.077062 \\
\hline 38 & $\mathrm{H}$ & 0.001385 & 0.070289 \\
\hline 39 & $\mathrm{H}$ & -0.000322 & 0.109098 \\
\hline 40 & $\mathrm{H}$ & 0.000515 & 0.110116 \\
\hline 41 & $\mathrm{H}$ & -0.004830 & 0.103231 \\
\hline 42 & $\mathrm{H}$ & 0.001727 & 0.115143 \\
\hline 43 & $\mathrm{H}$ & -0.003961 & 0.127460 \\
\hline 44 & $\mathrm{H}$ & -0.001853 & 0.089122 \\
\hline 45 & $\mathrm{H}$ & 0.001486 & 0.137418 \\
\hline
\end{tabular}




\begin{tabular}{|l|l|l|l|}
46 & $\mathrm{H}$ & 0.001917 & 0.106192 \\
47 & $\mathrm{H}$ & 0.000482 & 0.087723 \\
48 & $\mathrm{H}$ & -0.001825 & 0.089148 \\
49 & $\mathrm{H}$ & 0.000446 & 0.088006 \\
50 & $\mathrm{H}$ & 0.001415 & 0.139783 \\
51 & $\mathrm{H}$ & 0.001926 & 0.107075 \\
52 & $\mathrm{H}$ & -0.005116 & 0.103385 \\
53 & $\mathrm{H}$ & 0.000960 & 0.111472 \\
54 & $\mathrm{H}$ & 0.001647 & 0.114288 \\
55 & $\mathrm{H}$ & -0.003299 & 0.127873 \\
56 & $\mathrm{H}$ & -0.000990 & 0.109097 \\
\hline \hline
\end{tabular}

Table S16: Mulliken local spin densities for both spin states of $\left[\mathrm{Co}_{2}\left(\eta^{5}-\mathrm{Cp}\right)_{2}\left(\mu-\eta^{5}-1,8-\mathrm{DCN}\right)\right]$ calculated with B3LYP. The values are given in a.u.. Basis set: def-TZVP.

\begin{tabular}{|c|c|c|c|}
\hline \hline Number & Element & Spin density (high-spin) & Spin density (BS solution) \\
\hline 1 & $\mathrm{C}$ & -0.042036 & -0.039213 \\
2 & $\mathrm{C}$ & 0.108139 & 0.104674 \\
3 & $\mathrm{C}$ & -0.007269 & -0.007301 \\
\hline
\end{tabular}




\begin{tabular}{|c|c|c|c|}
\hline 4 & $\mathrm{C}$ & -0.068470 & -0.054170 \\
\hline 5 & $\mathrm{C}$ & 0.048382 & 0.047564 \\
\hline 6 & $\mathrm{Co}$ & 0.849370 & 0.832462 \\
\hline 7 & $\mathrm{C}$ & 0.063151 & 0.065749 \\
\hline 8 & $\mathrm{C}$ & -0.019827 & -0.015864 \\
\hline 9 & $\mathrm{C}$ & 0.001115 & -0.002718 \\
\hline 10 & $\mathrm{C}$ & -0.051522 & -0.052795 \\
\hline 11 & $\mathrm{C}$ & 0.084574 & 0.083530 \\
\hline 12 & $\mathrm{C}$ & -0.024332 & -0.008058 \\
\hline 13 & $\mathrm{C}$ & 0.024852 & -0.000283 \\
\hline 14 & $\mathrm{C}$ & 0.049176 & 0.025963 \\
\hline 15 & $\mathrm{C}$ & -0.024820 & 0.008654 \\
\hline 16 & $\mathrm{C}$ & 0.049921 & -0.027570 \\
\hline 17 & $\mathrm{C}$ & 0.108947 & -0.105095 \\
\hline 18 & $\mathrm{C}$ & -0.042748 & 0.039658 \\
\hline 19 & $\mathrm{C}$ & -0.067928 & 0.054588 \\
\hline 20 & $\mathrm{C}$ & 0.047388 & -0.047017 \\
\hline 21 & $\mathrm{C}$ & -0.006950 & 0.007526 \\
\hline 22 & $\mathrm{Co}$ & 0.848201 & -0.831881 \\
\hline
\end{tabular}




\begin{tabular}{|c|c|c|c|}
\hline 23 & $\mathrm{C}$ & 0.051031 & -0.053478 \\
\hline 24 & $\mathrm{C}$ & 0.016611 & -0.012824 \\
\hline 25 & $\mathrm{C}$ & -0.031443 & 0.027677 \\
\hline 26 & $\mathrm{C}$ & 0.090492 & -0.089753 \\
\hline 27 & $\mathrm{C}$ & -0.049017 & 0.050352 \\
\hline 28 & $\mathrm{C}$ & -0.024881 & -0.008185 \\
\hline 29 & $\mathrm{C}$ & 0.043411 & 0.023224 \\
\hline 30 & $\mathrm{C}$ & -0.028596 & 0.000467 \\
\hline 31 & $\mathrm{C}$ & 0.044356 & -0.024848 \\
\hline 32 & $\mathrm{C}$ & -0.025195 & 0.009024 \\
\hline 33 & $\mathrm{H}$ & -0.002092 & 0.001530 \\
\hline 34 & $\mathrm{H}$ & -0.002039 & -0.001447 \\
\hline 35 & $\mathrm{H}$ & 0.000949 & 0.000291 \\
\hline 36 & $\mathrm{H}$ & -0.002248 & -0.001264 \\
\hline 37 & $\mathrm{H}$ & -0.002268 & 0.001323 \\
\hline 38 & $\mathrm{H}$ & 0.000953 & -0.000319 \\
\hline 39 & $\mathrm{H}$ & -0.000290 & -0.000125 \\
\hline 40 & $\mathrm{H}$ & 0.000568 & 0.000418 \\
\hline 41 & $\mathrm{H}$ & -0.003943 & -0.003890 \\
\hline
\end{tabular}




\begin{tabular}{|c|c|c|c|}
\hline 42 & $\mathrm{H}$ & 0.001484 & 0.001516 \\
\hline 43 & $\mathrm{H}$ & -0.003204 & -0.003342 \\
\hline 44 & $\mathrm{H}$ & -0.001633 & -0.001516 \\
\hline 45 & $\mathrm{H}$ & 0.001555 & 0.001910 \\
\hline 46 & $\mathrm{H}$ & 0.001479 & 0.001111 \\
\hline 47 & $\mathrm{H}$ & 0.000320 & 0.000223 \\
\hline 48 & $\mathrm{H}$ & -0.001614 & 0.001489 \\
\hline 49 & $\mathrm{H}$ & 0.000296 & -0.000219 \\
\hline 50 & $\mathrm{H}$ & 0.001503 & -0.001961 \\
\hline 51 & $\mathrm{H}$ & 0.001481 & -0.001147 \\
\hline 52 & $\mathrm{H}$ & -0.004205 & 0.004164 \\
\hline 53 & $\mathrm{H}$ & 0.000948 & -0.000806 \\
\hline 54 & $\mathrm{H}$ & 0.001436 & -0.001470 \\
\hline 55 & $\mathrm{H}$ & -0.002658 & 0.002783 \\
\hline 56 & $\mathrm{H}$ & -0.000858 & 0.000689 \\
\hline
\end{tabular}

Table S17: Mulliken local spin densities for both spin states of $\left[\mathrm{Co}_{2}\left(\eta^{5}-\mathrm{Cp}\right)_{2}\left(\mu-\eta^{5}-1,8-\mathrm{DCN}\right)\right]$ calculated with LC- $\omega$ PBE. The values are given in a.u.. Basis set: def-TZVP. 


\begin{tabular}{|c|c|c|c|}
\hline Number & Element & Spin density (high-spin) & Spin density (BS solution) \\
\hline 1 & $\mathrm{C}$ & -0.059201 & -0.130808 \\
\hline 2 & $\mathrm{C}$ & 0.145691 & -0.194992 \\
\hline 3 & $\mathrm{C}$ & 0.003111 & -0.099871 \\
\hline 4 & $\mathrm{C}$ & -0.081226 & 0.005624 \\
\hline 5 & $\mathrm{C}$ & 0.059421 & -0.160842 \\
\hline 6 & $\mathrm{Co}$ & 0.824607 & -0.232082 \\
\hline 7 & $\mathrm{C}$ & 0.072975 & -0.144205 \\
\hline 8 & $\mathrm{C}$ & -0.024377 & -0.108150 \\
\hline 9 & $\mathrm{C}$ & 0.004366 & -0.147753 \\
\hline 10 & $\mathrm{C}$ & -0.055768 & -0.101594 \\
\hline 11 & $\mathrm{C}$ & 0.098809 & -0.161973 \\
\hline 12 & $\mathrm{C}$ & -0.104057 & 0.131630 \\
\hline 13 & $\mathrm{C}$ & 0.085619 & 0.111244 \\
\hline 14 & $\mathrm{C}$ & 0.111897 & -0.190304 \\
\hline 15 & $\mathrm{C}$ & -0.105759 & 0.136546 \\
\hline 16 & $\mathrm{C}$ & 0.113363 & -0.187417 \\
\hline 17 & $\mathrm{C}$ & 0.147797 & -0.184806 \\
\hline 18 & $\mathrm{C}$ & -0.060872 & -0.132608 \\
\hline
\end{tabular}




\begin{tabular}{|c|c|c|c|}
\hline 19 & $\mathrm{C}$ & -0.081292 & 0.002591 \\
\hline 20 & $\mathrm{C}$ & 0.058444 & -0.160645 \\
\hline 21 & $\mathrm{C}$ & 0.004279 & -0.100590 \\
\hline 22 & $\mathrm{Co}$ & 0.824042 & -0.231392 \\
\hline 23 & $\mathrm{C}$ & 0.059207 & -0.138889 \\
\hline 24 & $\mathrm{C}$ & 0.022743 & -0.154133 \\
\hline 25 & $\mathrm{C}$ & -0.038826 & -0.103373 \\
\hline 26 & $\mathrm{C}$ & 0.106829 & -0.165530 \\
\hline 27 & $\mathrm{C}$ & -0.053977 & -0.100342 \\
\hline 28 & $\mathrm{C}$ & -0.086695 & -0.107936 \\
\hline 29 & $\mathrm{C}$ & 0.105558 & -0.178146 \\
\hline 30 & $\mathrm{C}$ & -0.092038 & -0.071365 \\
\hline 31 & $\mathrm{C}$ & 0.106931 & -0.177577 \\
\hline 32 & $\mathrm{C}$ & -0.087535 & -0.110546 \\
\hline 33 & $\mathrm{H}$ & -0.005251 & 0.130774 \\
\hline 34 & $\mathrm{H}$ & -0.005182 & 0.130956 \\
\hline 35 & $\mathrm{H}$ & 0.003702 & 0.126668 \\
\hline 36 & $\mathrm{H}$ & -0.005337 & 0.140449 \\
\hline 37 & $\mathrm{H}$ & -0.005412 & 0.137055 \\
\hline
\end{tabular}




\begin{tabular}{|c|c|c|c|}
\hline 38 & $\mathrm{H}$ & 0.003722 & 0.126785 \\
\hline 39 & $\mathrm{H}$ & -0.000599 & 0.150624 \\
\hline 40 & $\mathrm{H}$ & 0.000638 & 0.151684 \\
\hline 41 & $\mathrm{H}$ & -0.004690 & 0.143827 \\
\hline 42 & $\mathrm{H}$ & 0.001518 & 0.156798 \\
\hline 43 & $\mathrm{H}$ & -0.003874 & 0.166836 \\
\hline 44 & $\mathrm{H}$ & -0.002099 & 0.136638 \\
\hline 45 & $\mathrm{H}$ & 0.002147 & 0.190854 \\
\hline 46 & $\mathrm{H}$ & 0.002046 & 0.159725 \\
\hline 47 & $\mathrm{H}$ & -0.000260 & 0.139969 \\
\hline 48 & $\mathrm{H}$ & -0.002108 & 0.136751 \\
\hline 49 & $\mathrm{H}$ & -0.000307 & 0.140249 \\
\hline 50 & $\mathrm{H}$ & 0.002110 & 0.193452 \\
\hline 51 & $\mathrm{H}$ & 0.002099 & 0.159988 \\
\hline 52 & $\mathrm{H}$ & -0.005045 & 0.143723 \\
\hline 53 & $\mathrm{H}$ & 0.001166 & 0.152795 \\
\hline 54 & $\mathrm{H}$ & 0.001488 & 0.155958 \\
\hline 55 & $\mathrm{H}$ & -0.003232 & 0.167280 \\
\hline 56 & $\mathrm{H}$ & -0.001305 & 0.150395 \\
\hline
\end{tabular}




\subsection{Mulliken local spin densities on all atoms for the high-spin structure of the meta- $\mathrm{C}_{8} \mathrm{H}_{8}$ diradical}

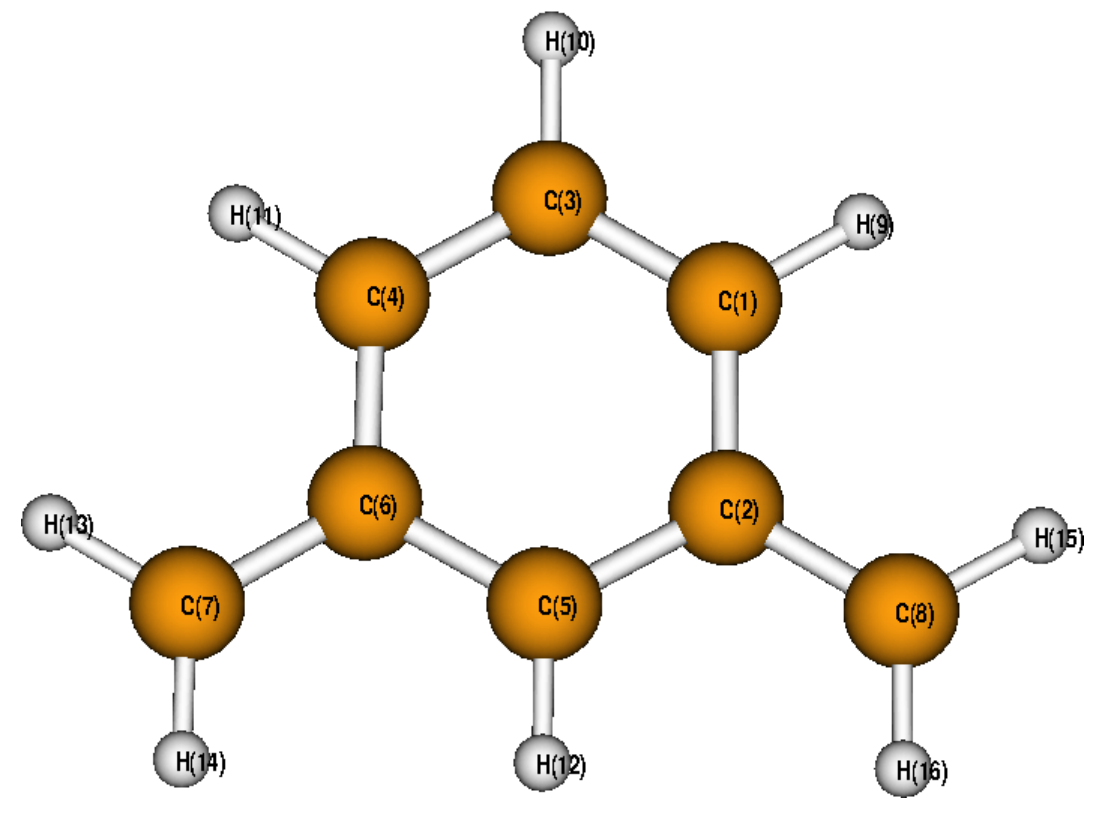

Figure S7: High-spin structure of the meta- $\mathrm{C}_{8} \mathrm{H}_{8}$ diradical and the corresponding atomic numbers as used for the atomic spin densities below. The same numbering scheme was used for the Broken-Symmetry solution.

Table S18: Mulliken local spin densities for both spin states of the meta- $\mathrm{C}_{8} \mathrm{H}_{8}$ diradical calculated with PBE.

The values are given in a.u.. Basis set: def-TZVP.

\begin{tabular}{|c|c|c|c|}
\hline \hline Number & Element & Spin density (high-spin) & Spin density (BS solution) \\
\hline 1 & $\mathrm{C}$ & 0.416558 & 0.029823 \\
2 & $\mathrm{C}$ & -0.251067 & 0.108626 \\
3 & $\mathrm{C}$ & -0.175102 & 0.002133 \\
\hline
\end{tabular}




\begin{tabular}{|c|c|c|c|}
\hline 4 & $\mathrm{C}$ & 0.417912 & -0.041470 \\
\hline 5 & $\mathrm{C}$ & 0.492948 & -0.002499 \\
\hline 6 & $\mathrm{C}$ & -0.252571 & -0.106504 \\
\hline 7 & $\mathrm{C}$ & 0.794673 & 0.830770 \\
\hline 8 & $\mathrm{C}$ & 0.785842 & -0.820324 \\
\hline 9 & $\mathrm{H}$ & -0.022131 & -0.003198 \\
\hline 10 & $\mathrm{H}$ & 0.006651 & -0.000082 \\
\hline 11 & $\mathrm{H}$ & -0.022308 & 0.003777 \\
\hline 12 & $\mathrm{H}$ & -0.022866 & 0.000200 \\
\hline 13 & $\mathrm{H}$ & -0.042865 & -0.044218 \\
\hline 14 & $\mathrm{H}$ & -0.041920 & -0.043625 \\
\hline 15 & $\mathrm{H}$ & -0.042002 & 0.043247 \\
\hline 16 & $\mathrm{H}$ & -0.041753 & 0.043344 \\
\hline
\end{tabular}

Table S19: Mulliken local spin densities for both spin states of the meta- $\mathrm{C}_{8} \mathrm{H}_{8}$ diradical calculated with BP86. The values are given in a.u.. Basis set: def-TZVP.

\begin{tabular}{|c|c|c|c|}
\hline \hline Number & Element & Spin density (high-spin) & Spin density (BS solution) \\
\hline 1 & $\mathrm{C}$ & 0.429848 & 0.030453 \\
2 & $\mathrm{C}$ & -0.264971 & 0.111837 \\
\hline
\end{tabular}




\begin{tabular}{|c|c|c|c|}
\hline 3 & $\mathrm{C}$ & -0.187528 & 0.002293 \\
\hline 4 & $\mathrm{C}$ & 0.431301 & -0.042368 \\
\hline 5 & $\mathrm{C}$ & 0.507266 & -0.002559 \\
\hline 6 & $\mathrm{C}$ & -0.266516 & -0.109525 \\
\hline 7 & $\mathrm{C}$ & 0.796851 & 0.833389 \\
\hline 8 & $\mathrm{C}$ & 0.788036 & -0.822949 \\
\hline 9 & $\mathrm{H}$ & -0.022538 & -0.004450 \\
\hline 10 & $\mathrm{H}$ & 0.006828 & -0.000082 \\
\hline 11 & $\mathrm{H}$ & -0.022722 & 0.005056 \\
\hline 12 & $\mathrm{H}$ & -0.022098 & 0.000209 \\
\hline 13 & $\mathrm{H}$ & -0.044125 & -0.045538 \\
\hline 14 & $\mathrm{H}$ & -0.043291 & -0.045141 \\
\hline 15 & $\mathrm{H}$ & -0.043306 & 0.044610 \\
\hline 16 & $\mathrm{H}$ & -0.043038 & 0.044765 \\
\hline
\end{tabular}

Table S20: Mulliken local spin densities for both spin states of the meta- $\mathrm{C}_{8} \mathrm{H}_{8}$ diradical calculated with TPSSH. The values are given in a.u.. Basis set: defTZVP.

\begin{tabular}{|l|l|l|l|}
\hline \hline Number & Element & Spin density (high-spin) & Spin density (BS solution) \\
\hline
\end{tabular}




\begin{tabular}{|c|c|c|c|}
\hline 1 & $\mathrm{C}$ & 0.484619 & 0.016712 \\
\hline 2 & $\mathrm{C}$ & -0.343456 & 0.141300 \\
\hline 3 & $\mathrm{C}$ & -0.265071 & 0.003692 \\
\hline 4 & $\mathrm{C}$ & 0.486062 & -0.031802 \\
\hline 5 & $\mathrm{C}$ & 0.561133 & -0.003582 \\
\hline 6 & $\mathrm{C}$ & -0.345884 & -0.136911 \\
\hline 7 & $\mathrm{C}$ & 0.850367 & 0.902334 \\
\hline 8 & $\mathrm{C}$ & 0.840812 & -0.891150 \\
\hline 9 & $\mathrm{H}$ & -0.026914 & -0.002613 \\
\hline 10 & $\mathrm{H}$ & 0.011396 & -0.000161 \\
\hline 11 & $\mathrm{H}$ & -0.027105 & 0.003426 \\
\hline 12 & $\mathrm{H}$ & -0.028020 & 0.000262 \\
\hline 13 & $\mathrm{H}$ & -0.050212 & -0.052943 \\
\hline 14 & $\mathrm{H}$ & -0.049371 & -0.052403 \\
\hline 15 & $\mathrm{H}$ & -0.049233 & 0.051827 \\
\hline 16 & $\mathrm{H}$ & -0.049122 & 0.052013 \\
\hline
\end{tabular}


Table S21: Mulliken local spin densities for both spin states of the meta- $\mathrm{C}_{8} \mathrm{H}_{8}$ diradical calculated with B3LYP. The values are given in a.u.. Basis set: def-TZVP.

\begin{tabular}{|c|c|c|c|}
\hline Number & Element & Spin density (high-spin) & Spin density (BS solution) \\
\hline 1 & $\mathrm{C}$ & 0.472552 & 0.017391 \\
\hline 2 & $\mathrm{C}$ & -0.329125 & 0.136131 \\
\hline 3 & $\mathrm{C}$ & -0.248740 & 0.003547 \\
\hline 4 & $\mathrm{C}$ & 0.473891 & -0.032565 \\
\hline 5 & $\mathrm{C}$ & 0.548101 & -0.003254 \\
\hline 6 & $\mathrm{C}$ & -0.331192 & -0.131756 \\
\hline 7 & $\mathrm{C}$ & 0.821474 & 0.872223 \\
\hline 8 & $\mathrm{C}$ & 0.812362 & -0.861336 \\
\hline 9 & $\mathrm{H}$ & -0.021561 & -0.002222 \\
\hline 10 & $\mathrm{H}$ & 0.009022 & -0.000131 \\
\hline 11 & $\mathrm{H}$ & -0.021730 & 0.002895 \\
\hline 12 & $\mathrm{H}$ & -0.021811 & 0.000201 \\
\hline 13 & $\mathrm{H}$ & -0.041506 & -0.043557 \\
\hline 14 & $\mathrm{H}$ & -0.040568 & -0.042916 \\
\hline 15 & $\mathrm{H}$ & -0.040672 & 0.042604 \\
\hline
\end{tabular}




\begin{tabular}{|l|l|l|l|}
16 & $\mathrm{H}$ & -0.040497 & 0.042744 \\
\hline
\end{tabular}

Table S22: Mulliken local spin densities for both spin states of the meta- $\mathrm{C}_{8} \mathrm{H}_{8}$ diradical calculated with LC$\omega \mathrm{PBE}$. The values are given in a.u.. Basis set: def-TZVP.

\begin{tabular}{|c|c|c|c|}
\hline Number & Element & Spin density (high-spin) & Spin density (BS solution) \\
\hline 1 & $\mathrm{C}$ & 0.637093 & -0.010974 \\
\hline 2 & $\mathrm{C}$ & -0.555793 & 0.220786 \\
\hline 3 & $\mathrm{C}$ & -0.449640 & 0.010032 \\
\hline 4 & $\mathrm{C}$ & 0.638698 & -0.019099 \\
\hline 5 & $\mathrm{C}$ & 0.707270 & -0.008667 \\
\hline 6 & $\mathrm{C}$ & -0.559537 & -0.204326 \\
\hline 7 & $\mathrm{C}$ & 0.940321 & 1.018873 \\
\hline 8 & $\mathrm{C}$ & 0.930258 & -1.006424 \\
\hline 9 & $\mathrm{H}$ & -0.030639 & -0.000528 \\
\hline 10 & $\mathrm{H}$ & 0.018295 & -0.000413 \\
\hline 11 & $\mathrm{H}$ & -0.030814 & 0.001977 \\
\hline 12 & $\mathrm{H}$ & -0.031277 & 0.000411 \\
\hline 13 & $\mathrm{H}$ & -0.054409 & -0.058854 \\
\hline 14 & $\mathrm{H}$ & -0.053321 & -0.058084 \\
\hline
\end{tabular}




\begin{tabular}{|l|l|l|l|}
15 & $\mathrm{H}$ & -0.053335 & 0.057541 \\
16 & $\mathrm{H}$ & -0.053169 & 0.057750 \\
\hline
\end{tabular}

\section{Structural differences between spin states}

Table S23: Structural parameters of the spin-state structures of the $\left[\mathrm{Mn}_{2}(\mathrm{Ac})_{4}\left(\mathrm{H}_{2} \mathrm{O}\right)_{2}\right]$ complex.

\begin{tabular}{|c|c|c|}
\hline \hline Parameter & hs structure & BS structure \\
\hline $\mathrm{R}(\mathrm{Mn}-\mathrm{Mn})$ & $3.024 \AA$ & $2.704 \AA$ \\
$\mathrm{R}(\mathrm{Mn}-\mathrm{O}[\mathrm{Ac}])$ & $2.124 \AA$ & $2.113 \AA$ \\
$\angle \mathrm{Mn}-\mathrm{O}[\mathrm{Ac}]-\mathrm{C}[\mathrm{Ac}]$ & $127.62^{\circ}$ & $123.86^{\circ}$ \\
\hline \hline
\end{tabular}

Table S24: Structural parameters of the spin-state structures of the $\left[\mathrm{Mn}_{2}(\mu-\mathrm{OMe})_{2}(\mathrm{HL})_{4}\right]$ complex.

\begin{tabular}{|c|c|c|}
\hline \hline Parameter & hs structure & BS structure \\
\hline $\mathrm{R}(\mathrm{Mn}-\mathrm{Mn})$ & $3.170 \AA$ & $3.134 \AA$ \\
$\angle \mathrm{Mn}-\mathrm{O}[\mathrm{OM}]-\mathrm{Mn}$ & $102.67^{\circ}$ & $104.78^{\circ}$ \\
\hline
\end{tabular}




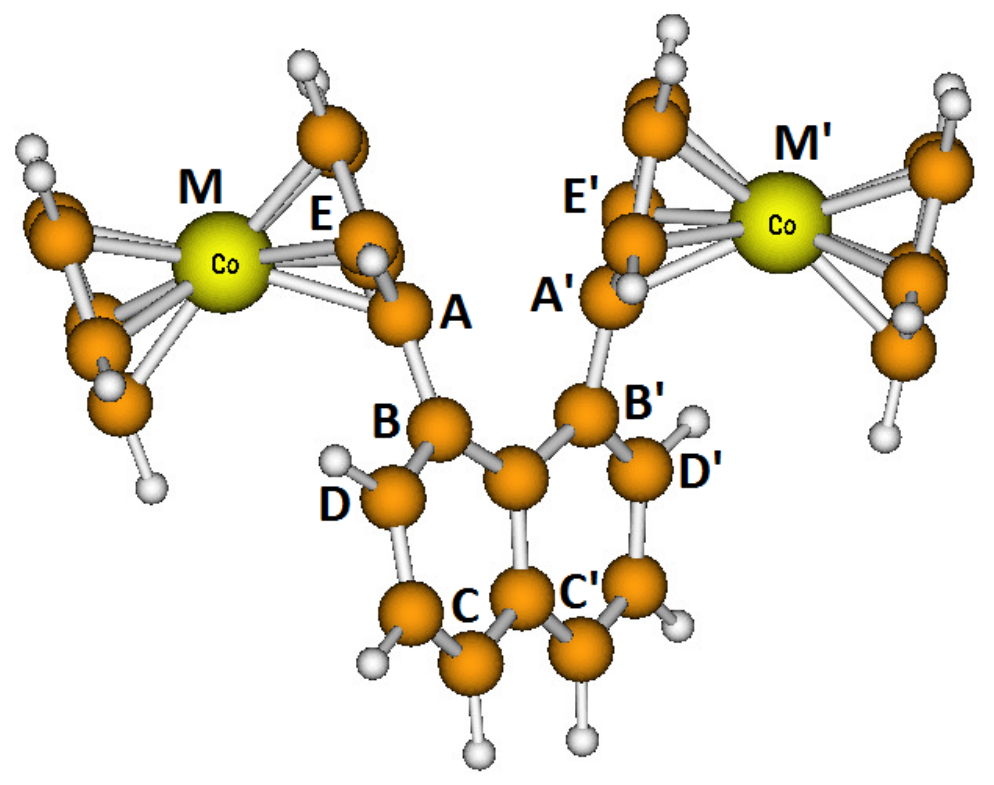

Figure S8: Structure of the $\left[\mathrm{Co}_{2}\left(\eta^{5}-\mathrm{Cp}\right)_{2}\left(\mu-\eta^{5}-1,8-\mathrm{DCN}\right)\right]$ complex with declarations of the atoms used to define the parameters given in Table S25. 
Table S25: Structural parameters of the $\left[\mathrm{Co}_{2}\left(\eta^{5}-\mathrm{Cp}\right)_{2}\left(\mu-\eta^{5}-1,8-\mathrm{DCN}\right)\right]$ and the $\left[\mathrm{V}_{2}\left(\eta^{5}-\mathrm{Cp}\right)_{2}\left(\mu-\eta^{5}-1,8-\mathrm{DCN}\right)\right]$ complex in both spin states. The atoms for the definition of parameters are declared in Figure S8.

\begin{tabular}{|c|cc|cc|}
\hline \hline & {$\left[\mathrm{Co}_{2}\left(\eta^{5}-\mathrm{Cp}\right)_{2}\left(\mu-\eta^{5}-1,8-\mathrm{DCN}\right)\right]$} & {$\left[\mathrm{V}_{2}\left(\eta^{5}-\mathrm{Cp}\right)_{2}\left(\mu-\eta^{5}-1,8-\mathrm{DCN}\right)\right]$} \\
\hline Parameter & hs structure & BS structure & hs structure & BS structure \\
\hline $\mathrm{R}\left(\mathrm{M}-\mathrm{M}^{\prime}\right)$ & $6.822 \AA$ & $6.865 \AA$ & $7.046 \AA$ & $7.046 \AA$ \\
$\mathrm{R}\left(\mathrm{A}-\mathrm{A}^{\prime}\right)$ & $2.943 \AA$ & $2.990 \AA$ & $2.955 \AA$ & $2.955 \AA$ \\
$\angle \mathrm{ABC}$ & $167.88 \AA$ & $167.89 \AA$ & $170.51 \AA$ & $170.52 \AA$ \\
$\angle \mathrm{A}^{\prime} \mathrm{B}^{\prime} \mathrm{C}^{\prime}$ & $168.43^{\circ}$ & $168.30^{\circ}$ & $170.51^{\circ}$ & $170.53^{\circ}$ \\
dihedral(DABE) & $35.54^{\circ}$ & $35.05^{\circ}$ & $45.26^{\circ}$ & $45.26^{\circ}$ \\
dihedral(DABE) & $36.99^{\circ}$ & $31.04^{\circ}$ & $45.27^{\circ}$ & $45.26^{\circ}$ \\
\hline \hline
\end{tabular}

Table S26: Structural parameters of the spin-state structures of the $\left[\mathrm{Cu}_{2}(\mathrm{Ac})_{4}\left(\mathrm{H}_{2} \mathrm{O}\right)_{2}\right]$ complex.

\begin{tabular}{|c|c|c|}
\hline \hline Parameter & hs structure & BS structure \\
\hline $\mathrm{R}(\mathrm{Cu}-\mathrm{Cu})$ & $2.514 \AA$ & $2.528 \AA$ \\
$\mathrm{R}(\mathrm{Cu}-\mathrm{O}[\mathrm{Ac}])$ & $2.034 \AA$ & $2.033 \AA$ \\
$\angle \mathrm{Cu}-\mathrm{O}[\mathrm{Ac}]-\mathrm{C}[\mathrm{Ac}]$ & $120.7^{\circ}$ & $120.6^{\circ}$ \\
\hline \hline
\end{tabular}




\section{Angular dependence of exchange coupling con- stants in $\left[\mathrm{Cu}_{2}(\mu-\mathrm{OH})_{2} \mathbf{C l}_{4}\right]^{2-}$}

Table S27: Mulliken local spins for the $\left[\mathrm{Cu}_{2}(\mu-\mathrm{OH})_{2} \mathrm{Cl}_{4}\right]^{2-}$ complex calculated with PBE and BP86. The values are given in a.u.. Basis set: def-TZVP.

\begin{tabular}{|c|c|c|c|c|}
\hline \multirow[b]{2}{*}{ Angle $\left(^{\circ}\right)$} & \multicolumn{2}{|c|}{$\mathrm{PBE}$} & \multicolumn{2}{|c|}{ BP86 } \\
\hline & high-spin state & BS solution & high-spin state & BS solution \\
\hline 80 & 0.226 & 0.202 & 0.225 & 0.199 \\
\hline 84 & 0.235 & 0.208 & 0.233 & 0.206 \\
\hline 86 & 0.238 & 0.210 & 0.237 & 0.207 \\
\hline 90 & 0.245 & 0.210 & 0.243 & 0.207 \\
\hline 92 & 0.247 & 0.208 & 0.246 & 0.206 \\
\hline 96 & 0.251 & 0.200 & 0.250 & 0.199 \\
\hline 98 & 0.253 & 0.198 & 0.251 & 0.194 \\
\hline 100 & 0.254 & 0.192 & 0.253 & 0.189 \\
\hline 102 & 0.255 & 0.186 & 0.254 & 0.182 \\
\hline 104 & 0.256 & 0.178 & 0.254 & 0.173 \\
\hline 106 & 0.256 & 0.169 & 0.255 & 0.164 \\
\hline 108 & 0.257 & 0.159 & 0.255 & 0.153 \\
\hline 110 & 0.257 & 0.147 & 0.255 & 0.141 \\
\hline
\end{tabular}


Table S28: Mulliken local spins for the $\left[\mathrm{Cu}_{2}(\mu-\mathrm{OH})_{2} \mathrm{Cl}_{4}\right]^{2-}$ complex calculated with TPSSH and B3LYP. The values are given in a.u.. Basis set: def-TZVP.

\begin{tabular}{|c|cc|cc|}
\hline \hline & \multicolumn{2}{|c|}{ TPSSH } & \multicolumn{2}{c|}{ B3LYP } \\
\hline Angle $\left(^{\circ}\right)$ & high-spin state & BS solution & high-spin state & BS solution \\
\hline 80 & 0.259 & 0.254 & 0.264 & 0.262 \\
84 & 0.268 & 0.262 & 0.274 & 0.271 \\
86 & 0.271 & 0.265 & 0.277 & 0.274 \\
90 & 0.278 & 0.270 & 0.284 & 0.279 \\
92 & 0.280 & 0.271 & 0.287 & 0.281 \\
96 & 0.285 & 0.273 & 0.291 & 0.284 \\
98 & 0.287 & 0.273 & 0.293 & 0.284 \\
100 & 0.288 & 0.272 & 0.294 & 0.285 \\
102 & 0.289 & 0.271 & 0.295 & 0.285 \\
108 & 0.290 & 0.270 & 0.296 & 0.284 \\
106 & 0.291 & 0.268 & 0.297 & 0.283 \\
104 & 0.291 & 0.264 & 0.298 & 0.281 \\
\hline \hline
\end{tabular}


Table S29: Mulliken local spins for the $\left[\mathrm{Cu}_{2}(\mu-\mathrm{OH})_{2} \mathrm{Cl}_{4}\right]^{2-}$ complex calculated with LC- $\omega$ PBE. The values are given in a.u.. Basis set: def-TZVP.

\begin{tabular}{|c|cc|}
\hline \hline & \multicolumn{2}{|c|}{ LC- $\omega$ PBE } \\
\hline Angle $\left(^{\circ}\right)$ & high-spin state & BS solution \\
\hline 80 & 0.273 & 0.273 \\
84 & 0.282 & 0.281 \\
86 & 0.285 & 0.284 \\
90 & 0.291 & 0.289 \\
92 & 0.294 & 0.291 \\
96 & 0.298 & 0.293 \\
98 & 0.299 & 0.294 \\
100 & 0.301 & 0.295 \\
102 & 0.302 & 0.295 \\
104 & 0.303 & 0.295 \\
106 & 0.304 & 0.294 \\
108 & 0.304 & 0.294 \\
110 & 0.304 & 0.293 \\
\hline \hline
\end{tabular}

\section{Comparison of the expressions for the on-site po- tentials in JGREEN}

As already noticed in the paper itself our definition of the on-site potentials is not unique. It is also possible to define the on-site potentials with one projection operator, 


$$
\begin{aligned}
\hat{V}_{A}^{\prime} & =\hat{p}_{A}\left(\hat{h}^{\alpha}-\hat{h}^{\beta}\right) \\
& =\sum_{\mu \in A}|\mu\rangle\langle\mu|\left(\hat{h}^{\alpha}-\hat{h}^{\beta}\right) .
\end{aligned}
$$

The main difference the definition here and that used in this work is that for the definition given here the sum over all on-site potentials of the atomic magnetic sites $A$ adds up to the total potential of the system (consistent with other local partitioning approaches such as population and local spin analysis), while the definition applied in this work neglects all mixed matrix elements with one basis function on the atoms of magnetic site $A$ and the second being localized on atoms not belonging to magnetic site $A$,

$$
\hat{V}=\sum_{A} \hat{V}_{A}^{\prime} \neq \sum_{A} \hat{V}_{A}
$$

By applying the second definition of the on-site potentials in Equation (1), we get an expression where one of the sums is not restricted to the basis functions centered on the magnetic site,

$$
\begin{aligned}
J_{\text {Green }}^{\text {unit }}(\mathrm{F})= & \frac{1}{4}\left[\sum_{i, j} \sum_{\pi, \mu \in A} \sum_{\kappa, \mu^{\prime} \in B} \frac{f_{i}^{\alpha}-f_{j}^{\beta}}{\epsilon_{j}^{\beta}-\epsilon_{i}^{\alpha}} C_{\pi i}^{\alpha}\left(F_{\mu \pi}^{\alpha}-F_{\mu \pi}^{\beta}\right) C_{\mu j}^{\beta *}\right. \\
& \left.C_{\mu^{\prime} i}^{\alpha *}\left(F_{\mu^{\prime} \kappa}^{\alpha}-F_{\mu^{\prime} \kappa}^{\beta}\right) C_{\kappa j}^{\beta}\right] .
\end{aligned}
$$

It can be easily seen that in contrast to the formula given in the main text, Equation 4 shows an asymmetry, which can result in a dependence on the choice which magnetic sites constitutes $A$ and which constitutes $B$. It can be seen in the following sections that this dependence is rather small and does not lead to serious problems for the systems under study. However those problems can be circumvented by multiplying the definition in Equation 1 from the right with a projection operator $\hat{p}_{V}$ containing all basis function of the system. 


\section{1 $\mathbf{H}-\mathrm{He}-\mathrm{H}$ model system}

Table S30: Magnetic exchange coupling constants in $\mathrm{kJ} / \mathrm{mol}$ calculated for the $\mathrm{H}-\mathrm{He}-\mathrm{H}$ system by applying the on-site potentials with two projection operators $\left(\mathrm{J}_{\text {Green }}(\mathrm{F})\right)$ and those with one projection operator $\left(\mathrm{J}_{\mathrm{AB}, \mathrm{Green}}(\mathrm{F})\right)$ for different exchange-correlation functionals (values in brackets result from interchanging the magnetic sites $A$ and $B$. Basis set: def-TZVP.

\begin{tabular}{|c|c|c|c|c|}
\hline \multicolumn{5}{|c|}{$1.25 \AA$} \\
\hline & $\hat{V}_{A}$ & $\hat{V}_{A}^{\prime}$ & $\hat{V}_{A}$ & $\hat{V}_{A}^{\prime}$ \\
\hline Functional & $\mathrm{J}_{\text {Green }}(\mathrm{F})$ & $\mathrm{J}_{\mathrm{AB}, \text { Green }}(\mathrm{F})\left(\mathrm{J}{ }_{\mathrm{BA}, \text { Green }}(\mathrm{F})\right)$ & $\mathrm{J}_{\text {Green }}(\mathrm{AF})$ & $\mathrm{J}_{\mathrm{AB}, \text { Green }}(\mathrm{AF})\left(\mathrm{J}_{\mathrm{BA}, \text { Green }}(\mathrm{AF})\right)$ \\
\hline PBE & -54.44 & $-57.33(-57.33)$ & -33.22 & $-36.79(-33.88)$ \\
\hline BP86 & -75.30 & $-75.30(-75.30)$ & -36.46 & $-40.33(-37.37)$ \\
\hline TPSSH & -86.62 & $-90.00(-90.00)$ & -37.47 & $-42.25(-37.18)$ \\
\hline B3LYP & -31.63 & $-40.53(-40.53)$ & -26.96 & $-34.13(-27.77)$ \\
\hline \multicolumn{5}{|c|}{$1.625 \AA$} \\
\hline & $\hat{V}_{A}$ & $\hat{V}_{A}^{\prime}$ & $\hat{V}_{A}$ & $\hat{V}_{A}^{\prime}$ \\
\hline Functional & $\mathrm{J}_{\text {Green }}(\mathrm{F})$ & $\mathrm{J}_{\mathrm{AB}, \text { Green }}(\mathrm{F})\left(\mathrm{J}_{\mathrm{BA}, \text { Green }}(\mathrm{F})\right)$ & $\mathrm{J}_{\text {Green }}(\mathrm{AF})$ & $\mathrm{J}_{\mathrm{AB}, \text { Green }}(\mathrm{AF})\left(\mathrm{J}_{\mathrm{BA}, \text { Green }}(\mathrm{AF})\right)$ \\
\hline PBE & -4.97 & $-5.50(-5.50)$ & -4.22 & $-4.92(-4.25)$ \\
\hline BP86 & -5.74 & $-5.69(-5.69)$ & -6.38 & $-6.74(-6.05)$ \\
\hline TPSSH & -6.71 & $-7.11(-7.11)$ & -5.13 & $-5.80(-4.91)$ \\
\hline B3LYP & -4.69 & $-4.97(-4.97)$ & -4.25 & $-5.26(-4.09)$ \\
\hline \multicolumn{5}{|c|}{$2 \AA$} \\
\hline & $\hat{V}_{A}$ & $\hat{V}_{A}^{\prime}$ & $\hat{V}_{A}$ & $\hat{V}_{A}^{\prime}$ \\
\hline Functional & $\mathrm{J}_{\text {Green }}(\mathrm{F})$ & $\mathrm{J}_{\mathrm{AB}, \text { Green }}(\mathrm{F})\left(\mathrm{J}_{\mathrm{BA}, \text { Green }}(\mathrm{F})\right)$ & $\mathrm{J}_{\text {Green }}(\mathrm{AF})$ & $\mathrm{J}_{\mathrm{AB}, \text { Green }}(\mathrm{AF})\left(\mathrm{J}_{\mathrm{BA}, \text { Green }}(\mathrm{AF})\right)$ \\
\hline PBE & -0.48 & $-0.60(-0.60)$ & -0.30 & $-0.33(-0.32)$ \\
\hline BP86 & -0.75 & $\begin{array}{r}54 \\
-0.83(-0.83)\end{array}$ & -0.72 & $-0.75(-0.70)$ \\
\hline TPSSH & -0.46 & $-0.48(-0.48)$ & -0.44 & $-0.48(-0.41)$ \\
\hline B3LYP & -0.56 & $-0.60(-0.60)$ & -0.52 & $-0.61(-0.49)$ \\
\hline
\end{tabular}




\subsection{Larger systems with localized spins}

Table S31: Magnetic exchange coupling constants in $\mathrm{kJ} / \mathrm{mol}$ calculated for the $\left[\mathrm{Mn}_{2}(\mathrm{Ac})_{4}\left(\mathrm{H}_{2} \mathrm{O}\right)_{2}\right]$ complex by applying the on-site potentials with two projection operators $\left(\mathrm{J}_{\text {Green }}(\mathrm{F})\right)$ and those with one projection operator $\left(\mathrm{J}_{\mathrm{AB}, \mathrm{Green}}(\mathrm{F})\right)$ for exchange-correlation potentials. Basis set: def-TZVP.

\begin{tabular}{|c|c|c|c|c|}
\hline \multicolumn{5}{|c|}{ high-spin structure } \\
\hline & $\hat{V}_{A}$ & $\hat{V}_{A}^{\prime}$ & $\hat{V}_{A}$ & $\hat{V}_{A}^{\prime}$ \\
\hline Functional & $\mathrm{J}_{\text {Green }}(\mathrm{F})$ & $\mathrm{J}_{\mathrm{AB}, \mathrm{Green}}(\mathrm{F})\left(\mathrm{J}_{\mathrm{BA}, \text { Green }}(\mathrm{F})\right)$ & $\mathrm{J}_{\text {Green }}(\mathrm{AF})$ & $\mathrm{J}_{\mathrm{AB}, \text { Green }}(\mathrm{AF})\left(\mathrm{J}_{\mathrm{BA}, \text { Green }}(\mathrm{AF})\right)$ \\
\hline $\mathrm{PBE}$ & -0.71 & $-0.61(-0.61)$ & -0.14 & $-0.13(-0.13)$ \\
\hline BP 86 & -0.64 & $-0.62(-0.62)$ & -0.12 & $-0.13(-0.13)$ \\
\hline TPSSH & -0.30 & $-0.28(-0.28)$ & -0.32 & $-0.31(-0.31)$ \\
\hline B3LYP & -0.37 & $-0.17(-0.17)$ & -0.48 & $-0.12(-0.12)$ \\
\hline $\mathrm{LC}-\omega \mathrm{PBE}$ & -0.19 & $0.49(0.49)$ & -0.51 & $-0.52(-0.57)$ \\
\hline \multicolumn{5}{|c|}{ Broken-Symmetry structure } \\
\hline & $\hat{V}_{A}$ & $\hat{V}_{A}^{\prime}$ & $\hat{V}_{A}$ & $\hat{V}_{A}^{\prime}$ \\
\hline Functional & $\mathrm{J}_{\text {Green }}(\mathrm{F})$ & $\mathrm{J}_{\mathrm{AB}, \text { Green }}(\mathrm{F})\left(\mathrm{J}_{\mathrm{BA}, \text { Green }}(\mathrm{F})\right)$ & $\mathrm{J}_{\text {Green }}(\mathrm{AF})$ & $\mathrm{J}_{\mathrm{AB}, \text { Green }}(\mathrm{AF})\left(\mathrm{J}_{\mathrm{BA}, \text { Green }}(\mathrm{AF})\right)$ \\
\hline PBE & -2.05 & $-1.77(-1.77)$ & -0.14 & $-0.18(-0.18)$ \\
\hline BP 86 & -2.05 & $-1.75(-1.75)$ & -0.12 & $-0.19(-0.20)$ \\
\hline TPSSH & -1.16 & $-0.79(-0.79)$ & -0.39 & $-0.38(-0.39)$ \\
\hline B3LYP & -0.99 & $-0.52(-0.52)$ & -0.63 & $-0.52(-0.53)$ \\
\hline $\mathrm{LC}-\omega \mathrm{PBE}$ & -0.46 & $-0.06(-0.06)$ & -0.70 & $-1.34(-1.23)$ \\
\hline
\end{tabular}


Table S32: Magnetic exchange coupling constants in $\mathrm{kJ} / \mathrm{mol}$ calculated for the $\left[\mathrm{Mn}_{2}(\mu-\mathrm{OMe})_{2}(\mathrm{HL})_{4}\right]$ complex by applying the on-site potentials with two projection operators $\left(\mathrm{J}_{\text {Green }}(\mathrm{F})\right)$ and those with one projection operator $\left(\mathrm{J}_{\mathrm{AB}, \mathrm{Green}}(\mathrm{F})\right)$ for different exchange--correlation potentials. Basis set: def-TZVP.

\begin{tabular}{|c|c|c|c|c|}
\hline \multicolumn{5}{|c|}{ high-spin structure } \\
\hline & $\hat{V}_{A}$ & $\hat{V}_{A}^{\prime}$ & $\hat{V}_{A}$ & $\hat{V}_{A}^{\prime}$ \\
\hline Functional & $\mathrm{J}_{\text {Green }}(\mathrm{F})$ & $\mathrm{J}_{\mathrm{AB}, \text { Green }}(\mathrm{F})\left(\mathrm{J}_{\mathrm{BA}, \text { Green }}(\mathrm{F})\right)$ & $\mathrm{J}_{\text {Green }}(\mathrm{AF})$ & $\mathrm{J}_{\mathrm{AB}, \mathrm{Green}}(\mathrm{AF})\left(\mathrm{J}_{\mathrm{BA}, \text { Green }}(\mathrm{AF})\right)$ \\
\hline $\mathrm{PBE}$ & 0.18 & $0.18(0.18)$ & -0.05 & $-0.07(-0.07)$ \\
\hline BP 86 & 0.14 & $0.14(0.13)$ & -0.03 & $-0.05(-0.05)$ \\
\hline TPSSH & 0.18 & $0.27(0.25)$ & -0.07 & $-0.09(-0.09)$ \\
\hline B3LYP & 0.15 & $0.29(0.25)$ & -0.03 & $-0.03(-0.03)$ \\
\hline $\mathrm{LC}-\omega \mathrm{PBE}$ & 0.13 & $0.59(0.47)$ & -0.02 & $-0.02(-0.02)$ \\
\hline \multicolumn{5}{|c|}{ Broken-Symmetry structure } \\
\hline & $\hat{V}_{A}$ & $\hat{V}_{A}^{\prime}$ & $\hat{V}_{A}$ & $\hat{V}_{A}^{\prime}$ \\
\hline Functional & $\mathrm{J}_{\text {Green }}(\mathrm{F})$ & $\mathrm{J}_{\mathrm{AB}, \text { Green }}(\mathrm{F})\left(\mathrm{J}_{\mathrm{BA}, \text { Green }}(\mathrm{F})\right)$ & $\mathrm{J}_{\text {Green }}(\mathrm{AF})$ & $\mathrm{J}_{\mathrm{AB}, \text { Green }}(\mathrm{AF})\left(\mathrm{J}_{\mathrm{BA}, \text { Green }}(\mathrm{AF})\right)$ \\
\hline $\mathrm{PBE}$ & 0.03 & $0.02(0.02)$ & -0.05 & $-0.07(-0.06)$ \\
\hline BP86 & -6.18 & $-6.13(-6.13)$ & -0.11 & $-0.18(-0.19)$ \\
\hline TPSSH & 0.10 & $0.17(0.15)$ & -0.06 & $-0.08(-0.08)$ \\
\hline B3LYP & 0.09 & $0.20(0.17)$ & -0.04 & $-0.04(-0.04)$ \\
\hline $\mathrm{LC}-\omega \mathrm{PBE}$ & 0.05 & $0.11(0.09)$ & -0.02 & $-0.02(-0.02)$ \\
\hline
\end{tabular}


Table S33: Magnetic exchange coupling constants in $\mathrm{kJ} / \mathrm{mol}$ calculated for the $\left[\mathrm{V}_{2}\left(\eta^{5}-\mathrm{Cp}\right)_{2}\left(\mu-\eta^{5}-1,8-\mathrm{DCN}\right)\right]$ complex by applying the on-site potentials with two projection operators $\left(\mathrm{J}_{\text {Green }}(\mathrm{F})\right)$ and those with one projection operator $\left(\mathrm{J}_{\mathrm{AB}, \mathrm{Green}}\right.$ (F)) for different exchange-correlation potentials. Basis set: def-TZVP.

\begin{tabular}{|c|c|c|c|c|}
\hline \multicolumn{5}{|c|}{ high-spin structure } \\
\hline & $\hat{V}_{A}$ & $\hat{V}_{A}^{\prime}$ & $\hat{V}_{A}$ & $\hat{V}_{A}^{\prime}$ \\
\hline Functional & $\mathrm{J}_{\text {Green }}(\mathrm{F})$ & $\mathrm{J}_{\mathrm{AB}, \text { Green }}(\mathrm{F})\left(\mathrm{J}_{\mathrm{BA}, \text { Green }}(\mathrm{F})\right)$ & $\mathrm{J}_{\text {Green }}(\mathrm{AF})$ & $\mathrm{J}_{\mathrm{AB}, \mathrm{Green}}(\mathrm{AF})\left(\mathrm{J}_{\mathrm{BA}, \text { Green }}(\mathrm{AF})\right)$ \\
\hline PBE & $-6.28 \cdot 10^{-3}$ & $-8.42 \cdot 10^{-3}\left(-8.43 \cdot 10^{-3}\right)$ & -0.12 & $-0.16(-0.16)$ \\
\hline BP86 & $-6.34 \cdot 10^{-3}$ & $-8.43 \cdot 10^{-3}\left(-8.43 \cdot 10^{-3}\right)$ & -0.18 & $-0.25(-0.24)$ \\
\hline TPSSH & $-3.78 \cdot 10^{-3}$ & $-3.40 \cdot 10^{-3}\left(-3.40 \cdot 10^{-3}\right)$ & -0.32 & $-0.02(-0.02)$ \\
\hline B3LYP & $-1.98 \cdot 10^{-3}$ & $-8.75 \cdot 10^{-5}\left(-7.98 \cdot 10^{-5}\right)$ & -0.16 & $-0.01(-0.01)$ \\
\hline $\mathrm{LC}-\omega \mathrm{PBE}$ & $-4.27 \cdot 10^{-4}$ & $6.67 \cdot 10^{-3}\left(6.67 \cdot 10^{-3}\right)$ & -0.07 & $-4.44 \cdot 10^{-3}\left(-4.44 \cdot 10^{-3}\right)$ \\
\hline \multicolumn{5}{|c|}{ Broken-Symmetry structure } \\
\hline & $\hat{V}_{A}$ & $\hat{V}_{A}^{\prime}$ & $\hat{V}_{A}$ & $\hat{V}_{A}^{\prime}$ \\
\hline Functional & $\mathrm{J}_{\text {Green }}(\mathrm{F})$ & $\mathrm{J}_{\mathrm{AB}, \text { Green }}(\mathrm{F})\left(\mathrm{J}_{\mathrm{BA}, \text { Green }}(\mathrm{F})\right)$ & $\mathrm{J}_{\text {Green }}(\mathrm{AF})$ & $\mathrm{J}_{\mathrm{AB}, \text { Green }}(\mathrm{AF})\left(\mathrm{J}^{\prime}{ }_{\mathrm{BA}, \text { Green }}(\mathrm{AF})\right)$ \\
\hline PBE & $-8.55 \cdot 10^{-3}$ & $-8.55 \cdot 10^{-3}\left(-8.55 \cdot 10^{-3}\right)$ & -0.19 & $-0.20(-0.19)$ \\
\hline BP86 & $-8.59 \cdot 10^{-3}$ & $-8.60 \cdot 10^{-3}\left(-8.60 \cdot 10^{-3}\right)$ & -0.23 & $-0.24(-0.23)$ \\
\hline TPSSH & $-3.43 \cdot 10^{-3}$ & $-3.42 \cdot 10^{-3}\left(-3.43 \cdot 10^{-3}\right)$ & -0.25 & $-0.25(-0.25)$ \\
\hline B3LYP & $-1.99 \cdot 10^{-4}$ & $-2.14 \cdot 10^{-4}\left(-1.99 \cdot 10^{-4}\right)$ & -0.26 & $-0.26(-0.26)$ \\
\hline $\mathrm{LC}-\omega \mathrm{PBE}$ & $-4.38 \cdot 10^{-4}$ & $-6.23 \cdot 10^{-3}\left(-6.24 \cdot 10^{-3}\right)$ & -0.12 & $-0.21(-0.20)$ \\
\hline
\end{tabular}


Table S34: Magnetic exchange coupling constants in $\mathrm{kJ} / \mathrm{mol}$ calculated for the para$\mathrm{C}_{8} \mathrm{H}_{8}$ diradical by applying the on-site potentials with two projection operators $\left(\mathrm{J}_{\mathrm{Green}}\right.$ $(\mathrm{F})$ ) and those with one projection operator $\left(\mathrm{J}_{\mathrm{AB}, \mathrm{Green}}(\mathrm{F})\right)$ different exchangecorelation potentials. Basis set: def-TZVP.

\begin{tabular}{|c|c|c|c|c|}
\hline \hline & $\hat{V}_{A}$ & $\hat{V}_{A}^{\prime}$ & $\hat{V}_{A}$ & $\hat{V}_{A}^{\prime}$ \\
\hline Functional & $\mathrm{J}_{\text {Green }}(\mathrm{F})$ & $\mathrm{J}_{\text {AB,Green }}(\mathrm{F})\left(\mathrm{J}_{\text {BA,Green }}(\mathrm{F})\right)$ & $\mathrm{J}_{\text {Green }}(\mathrm{AF})$ & $\mathrm{J}_{\text {AB,Green }}(\mathrm{AF})\left(\mathrm{J}_{\text {BA,Green }}(\mathrm{AF})\right)$ \\
\hline PBE & -5.58 & $-5.53(-5.53)$ & -0.11 & $-0.12(-0.12)$ \\
BP86 & -5.74 & $-5.69(-5.69)$ & -0.10 & $-0.16(-0.16)$ \\
TPSSH & -1.78 & $-1.98(-1.98)$ & -0.09 & $-0.16(-0.16)$ \\
B3LYP & -1.08 & $-1.21(-1.22)$ & -0.10 & $-0.12(-0.12)$ \\
LC- $\omega$ PBE & -0.23 & $0.06(0.06)$ & -0.01 & $-0.04(-0.04)$ \\
\hline \hline
\end{tabular}




\subsection{Larger systems with delocalized spins}

Table S35: Magnetic exchange coupling constants in $\mathrm{kJ} / \mathrm{mol}$ calculated for the $\left[\mathrm{Cu}_{2}(\mathrm{Ac})_{4}\left(\mathrm{H}_{2} \mathrm{O}\right)_{2}\right]$ complex by applying the on-site potentials with two projection operators $\left(\mathrm{J}_{\text {Green }}(\mathrm{F})\right)$ and those with one projection operator $\left(\mathrm{J}_{\mathrm{AB}, \mathrm{Green}}(\mathrm{F})\right)$ for different exchange-correlation potentials. Basis set: def-TZVP.

\begin{tabular}{|c|c|c|c|c|}
\hline \multicolumn{5}{|c|}{ high-spin structure } \\
\hline & $\hat{V}_{A}$ & $\hat{V}_{A}^{\prime}$ & $\hat{V}_{A}$ & $\hat{V}_{A}^{\prime}$ \\
\hline Functional & $\mathrm{J}_{\text {Green }}(\mathrm{F})$ & $\mathrm{J}_{\mathrm{AB}, \mathrm{Green}}(\mathrm{F})\left(\mathrm{J}_{\mathrm{BA}, \text { Green }}(\mathrm{F})\right)$ & $\mathrm{J}_{\text {Green }}(\mathrm{AF})$ & $\mathrm{J}_{\mathrm{AB}, \mathrm{Green}}^{\prime}(\mathrm{AF})\left(\mathrm{J}_{\mathrm{BA}, \mathrm{Green}}^{\prime}(\mathrm{AF})\right)$ \\
\hline $\mathrm{PBE}$ & -5.58 & $-5.53(-5.53)$ & -0.11 & $-0.12(-0.12)$ \\
\hline BP86 & -5.74 & $-5.69(-5.69)$ & -0.10 & $-0.16(-0.16)$ \\
\hline TPSSH & -1.78 & $-1.98(-1.98)$ & -0.09 & $-0.16(-0.16)$ \\
\hline B3LYP & -1.08 & $-1.21(-1.22)$ & -0.10 & $-0.12(-0.12)$ \\
\hline $\mathrm{LC}-\omega \mathrm{PBE}$ & -0.23 & $0.06(0.06)$ & -0.01 & $-0.04(-0.04)$ \\
\hline \multicolumn{5}{|c|}{ Broken-Symmetry structure } \\
\hline & $\hat{V}_{A}$ & $\hat{V}_{A}^{\prime}$ & $\hat{V}_{A}$ & $\hat{V}_{A}^{\prime}$ \\
\hline Functional & $\mathrm{J}_{\text {Green }}(\mathrm{F})$ & $\mathrm{J}_{\mathrm{AB}, \text { Green }}(\mathrm{F})\left(\mathrm{J}_{\mathrm{BA}, \text { Green }}^{\prime}(\mathrm{F})\right)$ & $\mathrm{J}_{\text {Green }}(\mathrm{AF})$ & $\mathrm{J}_{\mathrm{AB}, \mathrm{Green}}(\mathrm{AF})\left(\mathrm{J}_{\mathrm{BA}, \mathrm{Green}}(\mathrm{AF})\right)$ \\
\hline $\mathrm{PBE}$ & -6.01 & $-5.95(-5.95)$ & -0.12 & $-0.13(-0.13)$ \\
\hline BP86 & -6.18 & $-6.13(-6.13)$ & -0.11 & $-0.18(-0.19)$ \\
\hline TPSSH & -1.86 & $-2.08(-2.08)$ & -0.13 & $-0.15(-0.14)$ \\
\hline B3LYP & -1.12 & $-1.27(-1.27)$ & -0.12 & $-0.17(-0.17)$ \\
\hline $\mathrm{LC}-\omega \mathrm{PBE}$ & -0.24 & $-0.06(-0.06)$ & -0.02 & $-0.32(-0.32)$ \\
\hline
\end{tabular}


Table S36: Magnetic exchange coupling constants in $\mathrm{kJ} / \mathrm{mol}$ calculated for the $\left[\mathrm{Co}_{2}\left(\eta^{5}-\mathrm{Cp}\right)_{2}\left(\mu-\eta^{5}-1,8-\mathrm{DCN}\right)\right]$ complex by applying the on-site potentials with two projection operators $\left(\mathrm{J}_{\text {Green }}(\mathrm{F})\right)$ and those with one projection operator $\left(\mathrm{J}_{\mathrm{AB}, \mathrm{Green}}\right.$ (F)) for different exchange-correlation potentials. Basis set: def-TZVP.

\begin{tabular}{|c|c|c|c|c|}
\hline \multicolumn{5}{|c|}{ high-spin structure } \\
\hline & $\hat{V}_{A}$ & $\hat{V}_{A}^{\prime}$ & $\hat{V}_{A}$ & $\hat{V}_{A}^{\prime}$ \\
\hline Functional & $\mathrm{J}_{\text {Green }}(\mathrm{F})$ & $\mathrm{J}_{\mathrm{AB}, \text { Green }}(\mathrm{F})\left(\mathrm{J}_{\mathrm{BA}, \text { Green }}(\mathrm{F})\right)$ & $\mathrm{J}_{\text {Green }}(\mathrm{AF})$ & $\mathrm{J}_{\mathrm{AB}, \mathrm{Green}}(\mathrm{AF})\left(\mathrm{J}_{\mathrm{BA}, \text { Green }}(\mathrm{AF})\right)$ \\
\hline $\mathrm{PBE}$ & 2.15 & $2.09(2.09)$ & -0.15 & $-0.13(-0.13)$ \\
\hline BP 86 & 1.95 & $1.92(1.92)$ & -0.13 & $-0.11(-0.11)$ \\
\hline TPSSH & 0.59 & $0.83(0.82)$ & -0.37 & $-0.32(-0.33)$ \\
\hline B3LYP & 0.39 & $0.63(0.63)$ & -1.13 & $-1.07(-1.07)$ \\
\hline $\mathrm{LC}-\omega \mathrm{PBE}$ & 0.11 & $0.41(0.41)$ & -0.01 & $-0.04(-0.04)$ \\
\hline \multicolumn{5}{|c|}{ Broken-Symmetry structure } \\
\hline & $\hat{V}_{A}$ & $\hat{V}_{A}^{\prime}$ & $\hat{V}_{A}$ & $\hat{V}_{A}^{\prime}$ \\
\hline Functional & $\mathrm{J}_{\text {Green }}(\mathrm{F})$ & $\mathrm{J}_{\mathrm{AB}, \text { Green }}(\mathrm{F})\left(\mathrm{J}_{\mathrm{BA}, \text { Green }}(\mathrm{F})\right)$ & $\mathrm{J}_{\text {Green }}(\mathrm{AF})$ & $\mathrm{J}_{\mathrm{AB}, \text { Green }}(\mathrm{AF})\left(\mathrm{J}_{\mathrm{BA}, \text { Green }}(\mathrm{AF})\right)$ \\
\hline PBE & -0.83 & $-0.83(-0.83)$ & -0.22 & $-0.18(-0.19)$ \\
\hline BP86 & -0.98 & $-0.98(-0.98)$ & -0.42 & $-0.36(-0.36)$ \\
\hline TPSSH & -0.25 & $-0.18(-0.18)$ & -1.98 & $-1.76(-1.79)$ \\
\hline B3LYP & -0.12 & $-0.03(-0.03)$ & -477.98 & $-0.52(-0.52)$ \\
\hline $\mathrm{LC}-\omega \mathrm{PBE}$ & -0.02 & $-0.06(-0.06)$ & -0.01 & $-0.02(-0.02)$ \\
\hline
\end{tabular}


Table S37: Magnetic exchange coupling constants in $\mathrm{kJ} / \mathrm{mol}$ calculated for the meta$\mathrm{C}_{8} \mathrm{H}_{8}$ diradical by applying the on-site potentials with two projection operators $\left(\mathrm{J}_{\text {Green }}\right.$ $(\mathrm{F}))$ and those with one projection operator $\left(\mathrm{J}_{\mathrm{AB}, \mathrm{Green}}(\mathrm{F})\right)$ for different exchangecorrelation potentials. Basis set: def-TZVP.

\begin{tabular}{|c|c|c|c|c|}
\hline \multicolumn{5}{|c|}{ high-spin structure } \\
\hline & $\hat{V}_{A}$ & $\hat{V}_{A}^{\prime}$ & $\hat{V}_{A}$ & $\hat{V}_{A}^{\prime}$ \\
\hline Functional & $\mathrm{J}_{\text {Green }}(\mathrm{F})$ & $\mathrm{J}_{\mathrm{AB}, \text { Green }}(\mathrm{F})\left(\mathrm{J}_{\mathrm{BA}, \text { Green }}(\mathrm{F})\right)$ & $\mathrm{J}_{\text {Green }}(\mathrm{AF})$ & $\mathrm{J}_{\mathrm{AB}, \mathrm{Green}}(\mathrm{AF})\left(\mathrm{J}_{\mathrm{BA}, \text { Green }}(\mathrm{AF})\right)$ \\
\hline $\mathrm{PBE}$ & 0.98 & $0.92(0.92)$ & -0.08 & $-0.05(-0.05)$ \\
\hline BP 86 & 0.97 & $0.91(0.91)$ & -0.24 & $-0.24(-0.24)$ \\
\hline TPSSH & 1.22 & $1.39(1.39)$ & -0.36 & $-0.34(-0.32)$ \\
\hline B3LYP & 1.21 & $1.59(1.59)$ & -0.43 & $-0.50(-0.47)$ \\
\hline $\mathrm{LC}-\omega \mathrm{PBE}$ & 1.68 & $3.09(3.09)$ & 4.03 & $3.04(2.28)$ \\
\hline \multicolumn{5}{|c|}{ Broken-Symmetry structure } \\
\hline & $\hat{V}_{A}$ & $\hat{V}_{A}^{\prime}$ & $\hat{V}_{A}$ & $\hat{V}_{A}^{\prime}$ \\
\hline Functional & $\mathrm{J}_{\text {Green }}(\mathrm{F})$ & $\mathrm{J}_{\mathrm{AB}, \text { Green }}(\mathrm{F})\left(\mathrm{J}_{\mathrm{BA}, \text { Green }}(\mathrm{F})\right)$ & $\mathrm{J}_{\text {Green }}(\mathrm{AF})$ & $\mathrm{J}_{\mathrm{AB}, \text { Green }}(\mathrm{AF})\left(\mathrm{J}_{\mathrm{BA}, \text { Green }}(\mathrm{AF})\right)$ \\
\hline PBE & 0.91 & $0.85(0.85)$ & 1.05 & $0.99(0.99)$ \\
\hline BP 86 & 0.91 & $0.85(0.85)$ & 1.04 & $0.99(0.99)$ \\
\hline TPSSH & 1.16 & $1.30(1.30)$ & 0.98 & $0.83(0.95)$ \\
\hline B3LYP & 1.16 & $1.49(1.49)$ & 0.79 & $0.51(0.76)$ \\
\hline $\mathrm{LC}-\omega \mathrm{PBE}$ & 1.61 & $2.87(2.87)$ & 0.47 & $0.11(0.43)$ \\
\hline
\end{tabular}




\section{Non-collinear DFT calculations}

Table S38: Coupling constants $J$ for the high-spin structures of all compounds (except for the $\left[\mathrm{Cu}_{2}(\mathrm{Ac})_{4}\left(\mathrm{H}_{2} \mathrm{O}\right)_{2}\right]$ complex and the dihydrogen molecule) obtained with $\mathrm{PBE}$ by employing the OPENMX program and pseudopotentials provided by the program for the core electrons. The coupling constants obtained with PBE/def-TZVP by employing the Gaussian09 program are given for comparison. The values are given in $\mathrm{kJ} / \mathrm{mol}$.

\begin{tabular}{|c|c|c|}
\hline \multicolumn{3}{|c|}{$\mathrm{H}-\mathrm{He}-\mathrm{H}$ model system } \\
\hline Compound & $J^{\text {OPENMX }}$ & $J^{\text {Gaussiano9 }}$ \\
\hline $\mathrm{H}-\mathrm{He}-\mathrm{H}(1.25 \AA)$ & -49.3 & -54.4 \\
\hline $\mathrm{H}-\mathrm{He}-\mathrm{H}(1.625 \AA)$ & -5.0 & -5.6 \\
\hline $\mathrm{H}-\mathrm{He}-\mathrm{H}(2 \AA)$ & -0.5 & -0.5 \\
\hline \multicolumn{3}{|c|}{ Larger systems with localized spins } \\
\hline$\left[\mathrm{Mn}_{2}(\mathrm{Ac})_{4}\left(\mathrm{H}_{2} \mathrm{O}\right)_{2}\right]$ & -0.77 & -0.78 \\
\hline$\left[\mathrm{Mn}_{2}(\mu-\mathrm{OMe})_{2}(\mathrm{HL})_{4}\right]$ & 0.32 & 0.30 \\
\hline$\left[\mathrm{V}_{2}\left(\eta^{5}-\mathrm{Cp}\right)_{2}\left(\mu-\eta^{5}-1,8-\mathrm{DCN}\right)\right]$ & -0.0074 & -0.0075 \\
\hline$\left[\mathrm{V}_{2}(\mu-\mathrm{O}-\mathrm{Me})_{2}(\mathrm{O})_{2}(\mathrm{ma})_{2}\right]$ & -2.57 & -2.59 \\
\hline para- $\mathrm{C}_{8} \mathrm{H}_{8}$ & -10.58 & -11.22 \\
\hline \multicolumn{3}{|c|}{ Larger systems with delocalized spins } \\
\hline$\left[\mathrm{Co}_{2}\left(\eta^{5}-\mathrm{Cp}\right)_{2}\left(\mu-\eta^{5}-1,8-\mathrm{DCN}\right)\right]$ & 2.23 & 1.82 \\
\hline meta- $\mathrm{C}_{8} \mathrm{H}_{8}$ & 19.74 & 19.07 \\
\hline
\end{tabular}




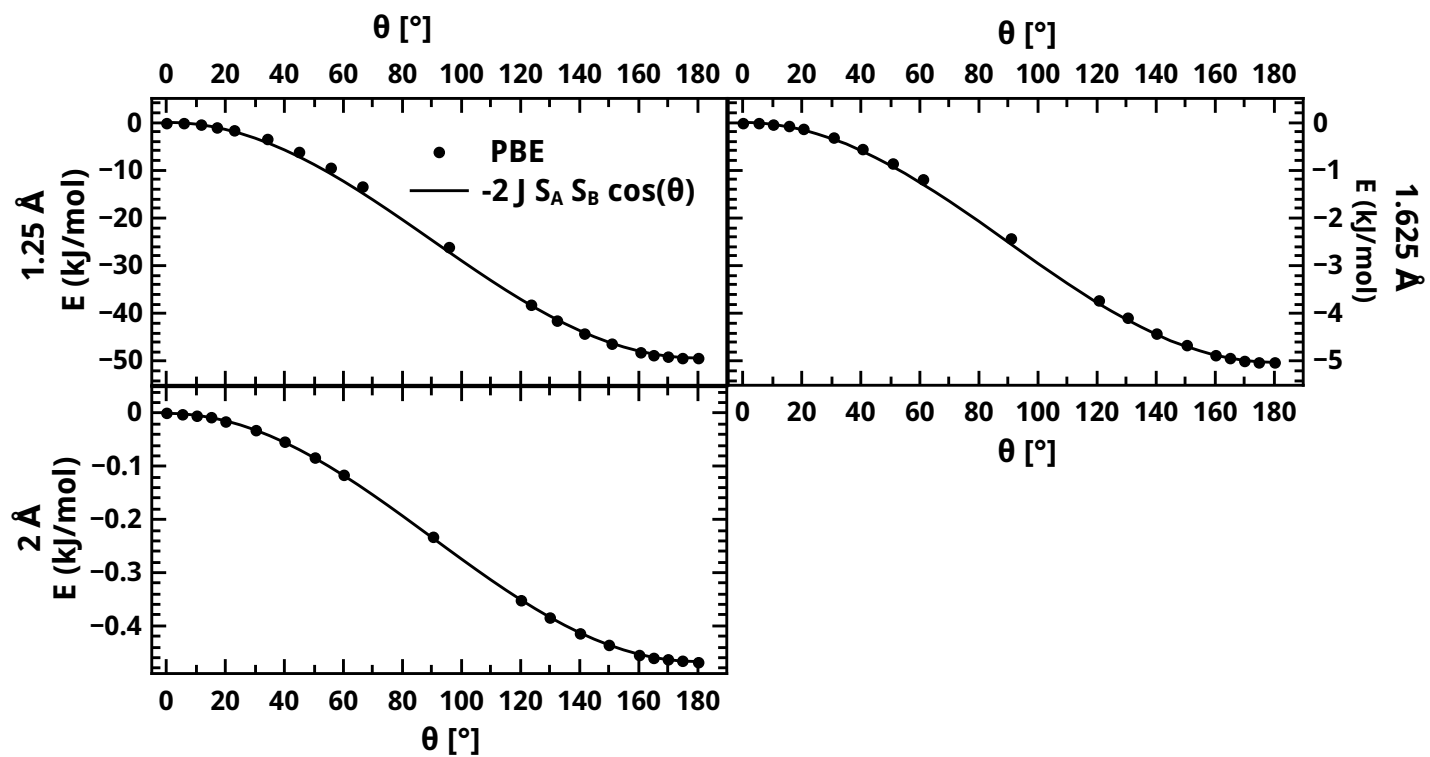

Figure S9: Non-collinear DFT calculations for the $\mathrm{H}-\mathrm{He}-\mathrm{H}$ system and different bond lengths performed with OPENMX employing the PBE functional (all other parameters are given in the Computational Methodology section). 


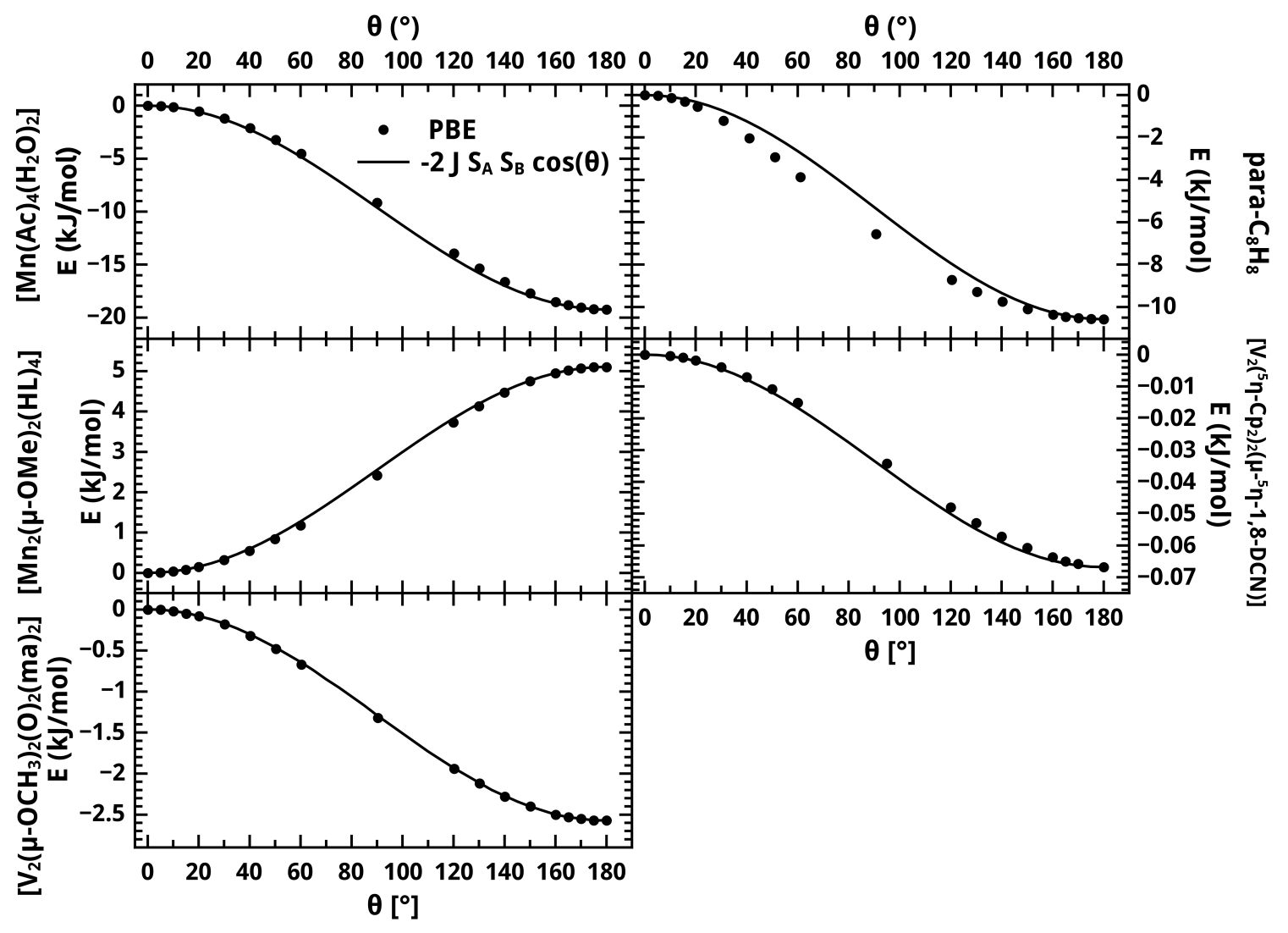

Figure S10: Non-collinear DFT calculations for the systems with localized spins in the high-spin state performed with OPENMX employing the PBE functional (other parameters are given in the Computational Methodology section).

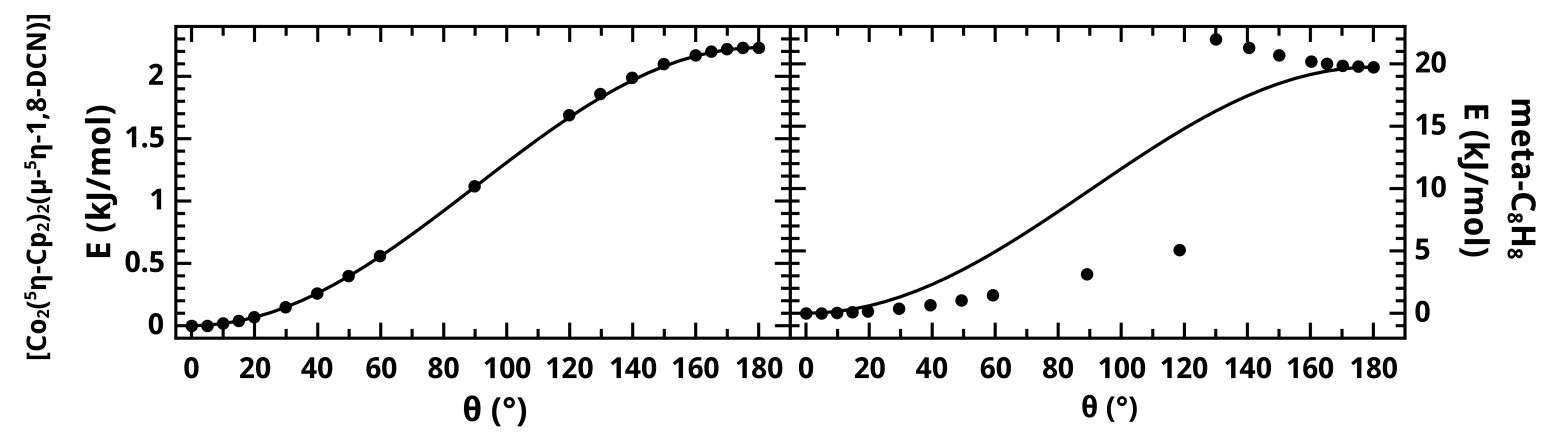

Figure S11: Non-collinear DFT calculations for the systems with delocalized spins in the high-spin state performed with OPENMX employing the PBE functional (other parameters are given in the Computational Methodology section). 


\section{Molecular structures and total energies}

\subsection{Localized spins}

$\left[\mathbf{M n}_{2}(\mathbf{A c})_{4}\left(\mathbf{H}_{2} \mathbf{O}\right)_{2}\right]$ (high-spin structure)

$E_{\text {tot }}=-3369.97403678454$ a.u.

Mn $0.002865 \quad 0.004900-2.847966$

$\begin{array}{llll}\text { Mn }-0.000142 & -0.000332 & 0.173436\end{array}$

C $-2.093281-3.632246-1.334794$

C $-1.329439-2.317173-1.343967$

$0-1.035747-1.808658-2.473910$

$00.005444 \quad 0.003287-5.047006$

$01.803092-1.053803-0.204843$

C $2.311770-1.343157-1.336501$

C $3.630962-2.099485-1.322682$

$0-0.001240-0.0003852 .375305$

$01.0374921 .813299-0.207167$

C $1.3319022 .321565-1.337584$

C $2.095716 \quad 3.636451-1.321554$

$\begin{array}{llll}0 & -1.803681 & 1.052838 & -0.205080\end{array}$

C $-2.3100871 .346181-1.336645$

C $-3.6307132 .100085-1.322819$

$0-1.037826-1.813176-0.211095$

$\begin{array}{llll}0 & -1.801645 & 1.058146 & -2.467933\end{array}$

$01.806610-1.049301-2.467701$

$\begin{array}{llll}0 & 1.040916 & 1.817291 & -2.470032\end{array}$

H $0.205053-0.7595382 .943869$

$\begin{array}{lllll}\text { H } & -0.204411 & 0.760630 & 2.942435\end{array}$

H $-2.296849-3.979451-2.353386$ 
H $-1.510997-4.390493-0.792415$

$\begin{array}{llll}\mathrm{H} & -3.039464 & -3.501157 & -0.790865\end{array}$

$\begin{array}{llll}\mathrm{H} & -3.982940 & 2.301740 & -2.340065\end{array}$

H $-4.3817551 .509464-0.779470$

H $-3.5069903 .046358-0.777294$

H $2.3016293 .987638 \quad-2.338317139$

H $1.5120824 .392448 \quad-0.777492$

H $3.0406253 .502994-0.775982$

H $3.982913-2.301683-2.339923$

H $4.383134-1.510115-0.779496$

H $3.505512-3.045394-0.776974$

H $0.201562-0.760601-5.612677$

H $-0.205503 \quad 0.760654-5.616259$

$\left[\mathrm{Mn}_{2}(\mathrm{Ac})_{4}\left(\mathbf{H}_{2} \mathrm{O}\right)_{2}\right]$ (BS structure) $E_{\mathrm{tot}}=-3369.97448056113$ a.u.
$\begin{array}{llll}\text { MN } & 0.003563 & 0.004352 & -2.689995\end{array}$
$\begin{array}{llll}\text { MN } & 0.000195 & 0.000221 & 0.013571\end{array}$
$\begin{array}{llll}\text { C } & -2.099811 & -3.648044 & -1.333915\end{array}$
C $\quad-1.335511-2.335731 \quad-1.346322$
$\begin{array}{llll}0 & -1.039317 & -1.822936 & -2.474238\end{array}$
$\begin{array}{llll}0 & 0.018472 & 0.007051 & -4.886910\end{array}$
$\begin{array}{llll}0 & 1.815079 & -1.059217 & -0.206351\end{array}$
C $\quad 2.328426 \quad-1.353931-1.335123$
$\begin{array}{llll}\text { C } & 3.644898 & -2.110923 & -1.321156\end{array}$
$\begin{array}{llll}0 & -0.005612 & -0.001570 & 2.210478\end{array}$
$\begin{array}{llll}0 & 1.040201 & 1.826385 & -0.208743\end{array}$
$\begin{array}{llll}\text { C } & 1.337907 & 2.340373 & -1.336417\end{array}$
$\begin{array}{llll}\text { C } & 2.102306 & 3.652500 & -1.319721\end{array}$ 


$$
\begin{array}{rrrr}
\mathrm{O} & -1.814183 & 1.059554 & -0.209011 \\
\mathrm{C} & -2.326555 & 1.356279 & -1.337561 \\
\mathrm{C} & -3.644102 & 2.111505 & -1.322600 \\
\mathrm{O} & -1.039280 & -1.825093 & -0.216869 \\
\mathrm{O} & -1.814084 & 1.063048 & -2.466384 \\
\mathrm{O} & 1.817782 & -1.057155 & -2.463653 \\
\mathrm{O} & 1.043089 & 1.830949 & -2.466038 \\
\mathrm{H} & 0.209257 & -0.760057 & 2.776087 \\
\mathrm{H} & -0.202955 & 0.762248 & 2.775328 \\
\mathrm{H} & -2.305546 & -3.996438 & -2.351562 \\
\mathrm{H} & -1.517374 & -4.406001 & -0.791417 \\
\mathrm{H} & -3.044813 & -3.515118 & -0.788466 \\
\mathrm{H} & -3.995651 & 2.315568 & -2.339502 \\
\mathrm{H} & -4.396467 & 1.521097 & -0.780895 \\
\mathrm{H} & -3.519089 & 3.056334 & -0.775048 \\
\mathrm{H} & 2.309397 & 4.003125 & -2.336350 \\
\mathrm{H} & 1.519180 & 4.409248 & -0.776313 \\
\mathrm{H} & 3.046616 & 3.518077 & -0.773473 \\
\mathrm{H} & 3.995489 & -2.314832 & -2.338442 \\
\mathrm{H} & 4.398384 & -1.521521 & -0.779923 \\
\mathrm{H} & 3.519035 & -3.055792 & -0.773925 \\
\mathrm{H} & 0.190863 & -0.764032 & -5.450137 \\
\hline & -0.213217 & 0.760297 & -5.452926
\end{array}
$$

$\left[\mathbf{M n}_{2}(\mu-\mathbf{O M e})_{2}(\mathbf{H L})_{4}\right]$ (high-spin structure) $E_{\text {tot }}=-8305.79402695095$ a.u.

$\begin{array}{lrrr}\text { MN } & 6.01562300 & -0.01441800 & 5.47594900 \\ \text { MN } & 5.20748300 & 1.65440500 & 2.90494700 \\ \text { S } & 8.10521000 & 3.19154700 & 6.01606700\end{array}$




\begin{tabular}{|c|c|c|c|}
\hline S & 7.04798300 & 3.57043700 & 8.82027400 \\
\hline S & 3.62935100 & -2.39179700 & 3.67820900 \\
\hline S & 5.61062400 & -4.67494000 & 3.77086800 \\
\hline S & 7.09393200 & 1.65369400 & -0.44357200 \\
\hline S & 7.77791900 & 4.58268300 & -0.15794800 \\
\hline S & 0.99644800 & 2.51892000 & 0.68859600 \\
\hline S & 2.27762900 & 4.05277900 & 2.94822600 \\
\hline 0 & 5.00866400 & -0.68506700 & 7.00887600 \\
\hline 0 & 4.38163900 & 1.55557400 & 10.54783600 \\
\hline $\mathrm{H}$ & 4.95786600 & 1.73242200 & 9.73164200 \\
\hline 0 & 7.75741200 & -0.69165700 & 6.02848100 \\
\hline 0 & 9.24927000 & -4.47283500 & 4.83200200 \\
\hline $\mathrm{H}$ & 8.34814000 & -4.03022500 & 4.68756600 \\
\hline 0 & 5.05826500 & 3.29436900 & 3.88838700 \\
\hline 0 & 6.77743700 & 6.94049800 & 2.57898100 \\
\hline $\mathrm{H}$ & 6.78457400 & 6.01456800 & 2.17043800 \\
\hline 0 & 5.28656200 & 0.17529900 & 1.68750700 \\
\hline 0 & 2.03452300 & -0.67742900 & -0.89560300 \\
\hline $\mathrm{H}$ & 2.17101900 & 0.06157900 & -0.22173500 \\
\hline 0 & 4.42157600 & 0.61979900 & 4.58480900 \\
\hline 0 & 6.79159100 & 0.92749400 & 3.91495300 \\
\hline $\mathrm{N}$ & 5.51290000 & 1.22145500 & 8.24064300 \\
\hline $\mathrm{N}$ & 6.25351400 & 1.59394500 & 7.13119500 \\
\hline $\mathrm{N}$ & 7.34984900 & -2.71406500 & 4.95616000 \\
\hline $\mathrm{N}$ & 6.11046000 & -2.16245000 & 4.67925000 \\
\hline $\mathrm{N}$ & 6.34096500 & 4.39472100 & 2.29734400 \\
\hline $\mathrm{N}$ & 6.37142500 & 3.15581700 & 1.67076400 \\
\hline $\mathrm{N}$ & 3.19488900 & 0.78717700 & 0.91600900 \\
\hline $\mathrm{N}$ & 3.34443600 & 1.83275400 & 1.82449200 \\
\hline
\end{tabular}




\begin{tabular}{|c|c|c|c|}
\hline $\mathrm{C}$ & 4.90473000 & 0.04585500 & 8.08494300 \\
\hline $\mathrm{C}$ & 4.06068700 & -0.44147400 & 9.18958100 \\
\hline $\mathrm{C}$ & 3.83120300 & 0.33870700 & 10.36152900 \\
\hline $\mathrm{C}$ & 2.99198500 & -0.16851600 & 11.37103800 \\
\hline $\mathrm{H}$ & 2.82851200 & 0.44689400 & 12.25654800 \\
\hline $\mathrm{C}$ & 2.39992100 & -1.41971600 & 11.23737900 \\
\hline $\mathrm{H}$ & 1.75532700 & -1.79505200 & 12.03488400 \\
\hline $\mathrm{C}$ & 2.62693800 & -2.19942400 & 10.09160200 \\
\hline $\mathrm{H}$ & 2.16690800 & -3.18334200 & 9.99363400 \\
\hline $\mathrm{C}$ & 3.44900800 & -1.70701800 & 9.08450400 \\
\hline $\mathrm{H}$ & 3.64522300 & -2.29081500 & 8.18498300 \\
\hline $\mathrm{C}$ & 7.00913700 & 2.63320000 & 7.29572800 \\
\hline $\mathrm{C}$ & 8.40240400 & 4.83154800 & 6.80427600 \\
\hline $\mathrm{H}$ & 9.34956800 & 5.21398900 & 6.40043800 \\
\hline $\mathrm{H}$ & 7.58461800 & 5.50932300 & 6.52645700 \\
\hline C & 8.47501300 & 4.62347200 & 8.30770200 \\
\hline $\mathrm{H}$ & 8.38696000 & 5.57515600 & 8.84932300 \\
\hline $\mathrm{H}$ & 9.40576200 & 4.12120600 & 8.60321000 \\
\hline $\mathrm{C}$ & 8.12575900 & -1.89012600 & 5.65843700 \\
\hline $\mathrm{C}$ & 9.47493100 & -2.35571000 & 6.01904800 \\
\hline $\mathrm{C}$ & 9.97376600 & -3.61966600 & 5.58467400 \\
\hline $\mathrm{C}$ & 11.27892400 & -4.00332400 & 5.94530400 \\
\hline $\mathrm{H}$ & 11.63916000 & -4.97372300 & 5.60191200 \\
\hline $\mathrm{C}$ & 12.07423300 & -3.16694600 & 6.72107300 \\
\hline $\mathrm{H}$ & 13.08252900 & -3.48662300 & 6.99193800 \\
\hline $\mathrm{C}$ & 11.59088000 & -1.92227300 & 7.15664500 \\
\hline $\mathrm{H}$ & 12.21540700 & -1.27060400 & 7.76874900 \\
\hline $\mathrm{C}$ & 10.30457700 & -1.52989200 & 6.80455500 \\
\hline $\mathrm{H}$ & 9.90160200 & -0.57145100 & 7.13179300 \\
\hline
\end{tabular}




\begin{tabular}{|c|c|c|c|}
\hline $\mathrm{C}$ & 5.25329100 & -2.95789800 & 4.12390100 \\
\hline $\mathrm{C}$ & 2.96635100 & -4.09234900 & 3.41410500 \\
\hline $\mathrm{H}$ & 2.63943700 & -4.50298000 & 4.37860300 \\
\hline $\mathrm{H}$ & 2.10102900 & -3.99628000 & 2.74433500 \\
\hline $\mathrm{C}$ & 4.06723100 & -4.93407900 & 2.79116500 \\
\hline $\mathrm{H}$ & 4.25638100 & -4.64324000 & 1.74940000 \\
\hline $\mathrm{H}$ & 3.83167500 & -6.00613400 & 2.83696000 \\
\hline $\mathrm{C}$ & 5.65601100 & 4.36921600 & 3.43480200 \\
\hline $\mathrm{C}$ & 5.54744400 & 5.61189400 & 4.21152300 \\
\hline $\mathrm{C}$ & 6.10501400 & 6.83870600 & 3.74352100 \\
\hline $\mathrm{C}$ & 5.95601800 & 8.00163700 & 4.52218900 \\
\hline $\mathrm{H}$ & 6.38910300 & 8.92843400 & 4.14423600 \\
\hline $\mathrm{C}$ & 5.27502100 & 7.96010800 & 5.73424200 \\
\hline $\mathrm{H}$ & 5.17148200 & 8.87477700 & 6.32129900 \\
\hline $\mathrm{C}$ & 4.72617600 & 6.75609000 & 6.20641000 \\
\hline $\mathrm{H}$ & 4.19981700 & 6.72330300 & 7.16058400 \\
\hline $\mathrm{C}$ & 4.86391800 & 5.60059700 & 5.44550700 \\
\hline $\mathrm{H}$ & 4.45543400 & 4.65176900 & 5.79281700 \\
\hline $\mathrm{C}$ & 8.38697500 & 2.32705400 & -1.57465100 \\
\hline $\mathrm{H}$ & 9.37337600 & 2.17223400 & -1.11771200 \\
\hline $\mathrm{H}$ & 8.32284200 & 1.75173900 & -2.50797500 \\
\hline $\mathrm{C}$ & 8.09847500 & 3.80088300 & -1.79951900 \\
\hline $\mathrm{H}$ & 7.21721800 & 3.94888900 & -2.43730100 \\
\hline $\mathrm{H}$ & 8.96009700 & 4.31875000 & -2.24214300 \\
\hline $\mathrm{C}$ & 6.99048400 & 3.13439600 & 0.52674700 \\
\hline $\mathrm{C}$ & 4.24498100 & -0.02259300 & 0.91343900 \\
\hline $\mathrm{C}$ & 4.24834100 & -1.17085100 & -0.00087200 \\
\hline $\mathrm{C}$ & 3.14659900 & -1.44009500 & -0.86510600 \\
\hline $\mathrm{C}$ & 3.20394900 & -2.55197100 & -1.72567100 \\
\hline
\end{tabular}




\begin{tabular}{|c|c|c|c|}
\hline $\mathrm{H}$ & 2.35104900 & -2.73734500 & -2.37961100 \\
\hline $\mathrm{C}$ & 4.32112800 & -3.37960900 & -1.73756900 \\
\hline $\mathrm{H}$ & 4.34694100 & -4.23434700 & -2.41635800 \\
\hline $\mathrm{C}$ & 5.40946000 & -3.12609100 & -0.88563300 \\
\hline $\mathrm{H}$ & 6.28298400 & -3.77866600 & -0.89491100 \\
\hline $\mathrm{C}$ & 5.36497400 & -2.03127200 & -0.03052100 \\
\hline $\mathrm{H}$ & 6.19146500 & -1.81663600 & 0.64629000 \\
\hline $\mathrm{C}$ & 2.35661900 & 2.68803900 & 1.82638200 \\
\hline $\mathrm{C}$ & -0.01392800 & 3.84834800 & 1.47628500 \\
\hline $\mathrm{H}$ & -0.67497900 & 4.25335900 & 0.69786000 \\
\hline $\mathrm{H}$ & -0.62170500 & 3.40194100 & 2.27420800 \\
\hline $\mathrm{C}$ & 0.93296300 & 4.90534400 & 2.01360600 \\
\hline $\mathrm{H}$ & 0.43478500 & 5.58012800 & 2.72260400 \\
\hline $\mathrm{H}$ & 1.38563600 & 5.49582100 & 1.20631200 \\
\hline $\mathrm{C}$ & 3.16070200 & 0.86403800 & 5.20013900 \\
\hline $\mathrm{H}$ & 2.91436200 & 0.04670200 & 5.89286200 \\
\hline $\mathrm{H}$ & 2.38414000 & 0.90717400 & 4.41940500 \\
\hline $\mathrm{H}$ & 3.16789900 & 1.81943400 & 5.75044800 \\
\hline $\mathrm{C}$ & 8.04710300 & 0.64410000 & 3.29821100 \\
\hline $\mathrm{H}$ & 8.78093100 & 0.38277500 & 4.07286800 \\
\hline $\mathrm{H}$ & 8.39989100 & 1.54056100 & 2.76533400 \\
\hline $\mathrm{H}$ & 7.95420300 & -0.18964300 & 2.58327700 \\
\hline
\end{tabular}

$\left[\mathbf{M n}_{2}(\mu-\mathrm{OMe})_{2}(\mathbf{H L})_{4}\right]$ (BS structure)

$E_{\text {tot }}=-8305.79309599647$ a.u.

$\begin{array}{lrrr}\text { MN } & 5.97560300 & -0.00394500 & 5.47446500 \\ \text { MN } & 5.20094100 & 1.62165500 & 2.90955500 \\ \text { S } & 8.08476400 & 3.20310800 & 6.01687700 \\ \text { S } & 7.05360300 & 3.57610800 & 8.83117000\end{array}$




\begin{tabular}{|c|c|c|c|}
\hline $\mathrm{S}$ & 3.58324800 & -2.39969300 & 3.67959000 \\
\hline S & 5.57581700 & -4.67364600 & 3.76681500 \\
\hline $\mathrm{S}$ & 7.10962800 & 1.69408800 & -0.48757300 \\
\hline$S$ & 7.80769300 & 4.61886100 & -0.18002300 \\
\hline$S$ & 1.04734900 & 2.49457300 & 0.71535100 \\
\hline$S$ & 2.34305900 & 4.04488300 & 2.95341900 \\
\hline 0 & 5.01075200 & -0.68244200 & 7.02381800 \\
\hline 0 & 4.39727500 & 1.54476000 & 10.57374400 \\
\hline $\mathrm{H}$ & 4.96684200 & 1.72780800 & 9.75435300 \\
\hline 0 & 7.71477300 & -0.67635700 & 6.00438900 \\
\hline 0 & 9.21877900 & -4.45577800 & 4.81637100 \\
\hline $\mathrm{H}$ & 8.31512500 & -4.01886600 & 4.67311300 \\
\hline 0 & 5.05925200 & 3.28520200 & 3.86571600 \\
\hline 0 & 6.77881600 & 6.93688700 & 2.57345300 \\
\hline $\mathrm{H}$ & 6.78807300 & 6.00894600 & 2.16448400 \\
\hline 0 & 5.30716700 & 0.12891200 & 1.70692500 \\
\hline 0 & 2.06779600 & -0.68772000 & -0.90236600 \\
\hline $\mathrm{H}$ & 2.20842300 & 0.05315600 & -0.23313400 \\
\hline 0 & 4.37672700 & 0.59959900 & 4.62152800 \\
\hline 0 & 6.75097900 & 0.94754200 & 3.89789600 \\
\hline $\mathrm{N}$ & 5.51212500 & 1.22595700 & 8.25556900 \\
\hline $\mathrm{N}$ & 6.24226300 & 1.60488500 & 7.14145100 \\
\hline $\mathrm{N}$ & 7.30872800 & -2.70619400 & 4.94500800 \\
\hline $\mathrm{N}$ & 6.06714200 & -2.15760000 & 4.67000900 \\
\hline $\mathrm{N}$ & 6.35171100 & 4.39743400 & 2.28279400 \\
\hline $\mathrm{N}$ & 6.39070400 & 3.17180200 & 1.63711400 \\
\hline $\mathrm{N}$ & 3.24066400 & 0.77521700 & 0.90881300 \\
\hline N & 3.40304900 & 1.81584300 & 1.82414200 \\
\hline $\mathrm{C}$ & 4.91013400 & 0.04728900 & 8.10177100 \\
\hline
\end{tabular}




\begin{tabular}{|c|c|c|c|}
\hline $\mathrm{C}$ & 4.07367200 & -0.44723000 & 9.20860000 \\
\hline $\mathrm{C}$ & 3.84776100 & 0.32794900 & 10.38451500 \\
\hline $\mathrm{C}$ & 3.01295900 & -0.18462300 & 11.39496800 \\
\hline $\mathrm{H}$ & 2.85196800 & 0.42728000 & 12.28336500 \\
\hline $\mathrm{C}$ & 2.42162600 & -1.43584800 & 11.25826700 \\
\hline $\mathrm{H}$ & 1.77977800 & -1.81481000 & 12.05622600 \\
\hline $\mathrm{C}$ & 2.64547900 & -2.21069600 & 10.10851600 \\
\hline $\mathrm{H}$ & 2.18613100 & -3.19467600 & 10.00776200 \\
\hline $\mathrm{C}$ & 3.46325600 & -1.71319400 & 9.10055700 \\
\hline $\mathrm{H}$ & 3.65661300 & -2.29308900 & 8.19789000 \\
\hline $\mathrm{C}$ & 6.99937900 & 2.64293200 & 7.30458500 \\
\hline $\mathrm{C}$ & 8.38323200 & 4.84366200 & 6.80240300 \\
\hline $\mathrm{H}$ & 9.32453300 & 5.23012600 & 6.38894300 \\
\hline $\mathrm{H}$ & 7.55945200 & 5.51772000 & 6.53342300 \\
\hline $\mathrm{C}$ & 8.47222600 & 4.63399500 & 8.30480700 \\
\hline $\mathrm{H}$ & 8.38723700 & 5.58480000 & 8.84837800 \\
\hline $\mathrm{H}$ & 9.40735400 & 4.13398600 & 8.59007600 \\
\hline $\mathrm{C}$ & 8.08457000 & -1.87826000 & 5.64038000 \\
\hline $\mathrm{C}$ & 9.43525600 & -2.33680300 & 6.00203200 \\
\hline $\mathrm{C}$ & 9.93929100 & -3.59922700 & 5.56904700 \\
\hline $\mathrm{C}$ & 11.24540600 & -3.97765600 & 5.93150400 \\
\hline $\mathrm{H}$ & 11.60965500 & -4.94691900 & 5.58910800 \\
\hline $\mathrm{C}$ & 12.03592400 & -3.13846500 & 6.70913400 \\
\hline $\mathrm{H}$ & 13.04460900 & -3.45481600 & 6.98250800 \\
\hline $\mathrm{C}$ & 11.54727100 & -1.89554500 & 7.14377400 \\
\hline $\mathrm{H}$ & 12.16795800 & -1.24197100 & 7.75779100 \\
\hline $\mathrm{C}$ & 10.26053000 & -1.50748300 & 6.78869400 \\
\hline $\mathrm{H}$ & 9.85377500 & -0.55036800 & 7.11507700 \\
\hline $\mathrm{C}$ & 5.21159600 & -2.95791300 & 4.11908400 \\
\hline
\end{tabular}




\begin{tabular}{|c|c|c|c|}
\hline $\mathrm{C}$ & 2.93041300 & -4.10277000 & 3.40350400 \\
\hline $\mathrm{H}$ & 2.60170000 & -4.51958000 & 4.36472800 \\
\hline $\mathrm{H}$ & 2.06741900 & -4.00792000 & 2.73056400 \\
\hline $\mathrm{C}$ & 4.03728100 & -4.93676300 & 2.78103200 \\
\hline $\mathrm{H}$ & 4.22879100 & -4.64137900 & 1.74100000 \\
\hline $\mathrm{H}$ & 3.80675100 & -6.01013200 & 2.82211200 \\
\hline $\mathrm{C}$ & 5.66186700 & 4.35938300 & 3.41831000 \\
\hline $\mathrm{C}$ & 5.55159300 & 5.59974700 & 4.20067900 \\
\hline $\mathrm{C}$ & 6.10607300 & 6.83002900 & 3.73665900 \\
\hline $\mathrm{C}$ & 5.95289300 & 7.99052500 & 4.51856100 \\
\hline $\mathrm{H}$ & 6.38333800 & 8.91946300 & 4.14275400 \\
\hline $\mathrm{C}$ & 5.27226200 & 7.94399500 & 5.73055300 \\
\hline $\mathrm{H}$ & 5.16592000 & 8.85668000 & 6.32025100 \\
\hline $\mathrm{C}$ & 4.72732600 & 6.73689900 & 6.19937000 \\
\hline $\mathrm{H}$ & 4.20181000 & 6.69975600 & 7.15387800 \\
\hline C & 4.86831900 & 5.58391700 & 5.43471200 \\
\hline $\mathrm{H}$ & 4.46273400 & 4.63290500 & 5.77958100 \\
\hline $\mathrm{C}$ & 8.41348800 & 2.36641200 & -1.60551000 \\
\hline $\mathrm{H}$ & 9.39629400 & 2.20588700 & -1.14279600 \\
\hline $\mathrm{H}$ & 8.35422200 & 1.79700700 & -2.54281200 \\
\hline C & 8.13253200 & 3.84325600 & -1.82387900 \\
\hline $\mathrm{H}$ & 7.25550800 & 3.99793900 & -2.46589600 \\
\hline $\mathrm{H}$ & 8.99871700 & 4.35885000 & -2.26026600 \\
\hline $\mathrm{C}$ & 7.01198000 & 3.16812000 & 0.49710300 \\
\hline $\mathrm{C}$ & 4.27640400 & -0.05262900 & 0.91626400 \\
\hline $\mathrm{C}$ & 4.27512700 & -1.19795700 & -0.00126200 \\
\hline $\mathrm{C}$ & 3.17422300 & -1.45906100 & -0.86832300 \\
\hline $\mathrm{C}$ & 3.22534100 & -2.57175800 & -1.72805200 \\
\hline $\mathrm{H}$ & 2.37292900 & -2.75138700 & -2.38423100 \\
\hline
\end{tabular}




\begin{tabular}{|c|c|c|c|}
\hline $\mathrm{C}$ & 4.33735400 & -3.40651100 & -1.73796300 \\
\hline $\mathrm{H}$ & 4.35918500 & -4.26135400 & -2.41676300 \\
\hline C & 5.42594400 & -3.15968600 & -0.88441800 \\
\hline $\mathrm{H}$ & 6.29597000 & -3.81687500 & -0.89305100 \\
\hline $\mathrm{C}$ & 5.38643700 & -2.06522700 & -0.02866800 \\
\hline $\mathrm{H}$ & 6.21316200 & -1.85551700 & 0.64932900 \\
\hline C & 2.41528300 & 2.67521300 & 1.83939000 \\
\hline $\mathrm{C}$ & 0.03666200 & 3.82131600 & 1.50718300 \\
\hline $\mathrm{H}$ & -0.63478600 & 4.21785800 & 0.73335900 \\
\hline $\mathrm{H}$ & -0.55989000 & 3.37460700 & 2.31330400 \\
\hline $\mathrm{C}$ & 0.98299700 & 4.88531400 & 2.02975800 \\
\hline $\mathrm{H}$ & 0.48972600 & 5.56034000 & 2.74204800 \\
\hline $\mathrm{H}$ & 1.42472900 & 5.47506500 & 1.21592800 \\
\hline $\mathrm{C}$ & 3.14414400 & 0.88497100 & 5.27367300 \\
\hline $\mathrm{H}$ & 2.88264600 & 0.07441600 & 5.96894200 \\
\hline $\mathrm{H}$ & 2.35237200 & 0.96612900 & 4.51127800 \\
\hline $\mathrm{H}$ & 3.19961900 & 1.83636300 & 5.82817200 \\
\hline $\mathrm{C}$ & 8.00368800 & 0.65740300 & 3.27404300 \\
\hline $\mathrm{H}$ & 8.73437100 & 0.38696700 & 4.04755400 \\
\hline $\mathrm{H}$ & 8.35766500 & 1.55363400 & 2.74297100 \\
\hline $\mathrm{H}$ & 7.89760700 & -0.17212600 & 2.55714100 \\
\hline
\end{tabular}

\footnotetext{
$\left[\mathbf{V}_{2}\left(\eta^{5}-\mathbf{C p}\right)_{2}\left(\mu-\eta^{5}-1,8-\mathbf{D C N}\right)\right]$ (high-spin structure $E_{\mathrm{tot}}=-3046.54454680307$ a.u.
}
$\begin{array}{llll}\mathrm{V} & 5.947147 & 4.491222 & 1.588750\end{array}$
C $8.073930 \quad 5.174880 \quad 1.996330$
C $\quad 7.613449 \quad 4.426498 \quad 3.125237$
$\begin{array}{llll}\text { C } & 7.359598 & 3.089628 & 2.705100\end{array}$
C $\quad 7.644734 \quad 3.002652 \quad 1.309059$ 


\begin{tabular}{|c|c|c|c|}
\hline & 8.077430 & 4.288745 & \\
\hline & 8.380212 & 4.563734 & -0.13779 \\
\hline & 3.939406 & 5.198068 & 2.38586 \\
\hline & 4.457847 & 6.200812 & 1.51165 \\
\hline & 4.763755 & .204034 & 1.79756 \\
\hline & 4.557208 & 5.636186 & 0.20368 \\
\hline & 4.925283 & 6.142980 & -0.68484 \\
\hline & 4.099173 & & 0.26913 \\
\hline & 3.717627 & 4.013631 & 1.61806 \\
\hline & 8.404318 & & $1 . \subseteq$ \\
\hline & 7.494128 & .822844 & \\
\hline & 6.993583 & & \\
\hline & 7.540171 & & \\
\hline & 3.763443 & & \\
\hline & 4.059513 & 3.582330 & -0.55996 \\
\hline & 3.338500 & 3.067773 & \\
\hline & 7.841482 & 7.362690 & \\
\hline & 9.190467 & 55 & \\
\hline & 7.235979 & 96 & \\
\hline & 7.948298 & & \\
\hline & 9.976569 & & \\
\hline & 9.190441 & & \\
\hline & 7.484037 & 9.31052 & \\
\hline & 8.576141 & 39152 & \\
\hline & 10.5 & 2655 & \\
\hline & 10.30696 & 5.1 & \\
\hline & 9.804747 & 9.439117 & \\
\hline & 8.608111 & 10.530889 & 1.921 \\
\hline & 11.144951 & 6.834890 & 0.14 \\
\hline
\end{tabular}



C 10.432622
8.766459
5.055325
V 12.433748
4.491232
4.339044
C 10.767459
4.426485
2.802565
C 10.303471
4.288757
5.058557
$\mathrm{H}$
9.772787
10.530917
4.006552
H 10.896840
9.315513
5.876743
C $\quad 11.021287$
3.089665
3.222697
C $\quad 10.736174$
3.002640
4.618734
H 10.000690
4.563747
6.065591
C 14.441492
5.198076
3.541897
C 13.923048
6.200812
4.416161
H 13.617149
7.204035
4.130231
C 13.823700
5.636164
5.724083
H 13.455611
6.142979
6.612648
C 14.281726
4.284322
5.658650
C 14.663288
4.013605
4.309726
H 10.886769
4.822852
1.799163
H 11.387318
2.283087
2.592773
H 10.840728
2.116963
5.240148
H 14.617458
5.310545
2.475132
H 14.321383
3.582317
6.487758
H 15.042395
3.067782
3.931171

$\left[\mathbf{V}_{2}\left(\eta^{5}-\mathbf{C p}\right)_{2}\left(\mu-\eta^{5}-1,8-\mathbf{D C N}\right)\right]$ (BS structure) $E_{\text {tot }}=-3046.54457552870$ a.u.

V

$\mathrm{C}$

C

C
5.94713900

8.07403600

7.61267600

7.35865400
4.49182000

5.17487200

4.42691100

3.08997700
1.58843300

1.99631300

3. 12515000

2.70499700 


\begin{tabular}{|c|c|c|c|}
\hline $\mathrm{C}$ & 7.64459800 & 3.00276500 & 1.30920700 \\
\hline C & 8.07788200 & 4.28852100 & 0.86928900 \\
\hline $\mathrm{H}$ & 8.38237800 & 4.56202600 & -0.13764200 \\
\hline $\mathrm{C}$ & 3.93924000 & 5.19868800 & 2.38612200 \\
\hline $\mathrm{C}$ & 4.45769700 & 6.20101500 & 1.51148200 \\
\hline $\mathrm{H}$ & 4.76351900 & 7.20435400 & 1.79703600 \\
\hline $\mathrm{C}$ & 4.55698600 & 5.63588400 & 0.20369600 \\
\hline $\mathrm{H}$ & 4.92530600 & 6.14235000 & -0.68491800 \\
\hline $\mathrm{C}$ & 4.09914600 & 4.28394000 & 0.26976100 \\
\hline $\mathrm{C}$ & 3.71771100 & 4.01378300 & 1.61888900 \\
\hline $\mathrm{C}$ & 8.40427100 & 6.61115900 & 1.95574800 \\
\hline $\mathrm{H}$ & 7.49272200 & 4.82400200 & 4.12814800 \\
\hline $\mathrm{H}$ & 6.99226900 & 2.28304200 & 3.33409200 \\
\hline $\mathrm{H}$ & 7.54178900 & 2.11664700 & 0.68818100 \\
\hline $\mathrm{H}$ & 3.76312400 & 5.31188200 & 3.45280100 \\
\hline $\mathrm{H}$ & 4.05948500 & 3.58155400 & -0.55902300 \\
\hline $\mathrm{H}$ & 3.33848900 & 3.06826400 & 1.99811100 \\
\hline $\mathrm{C}$ & 7.84171600 & 7.36260100 & 0.92585400 \\
\hline $\mathrm{C}$ & 9.19045300 & 7.27616200 & 2.96389600 \\
\hline $\mathrm{H}$ & 7.23535800 & 6.83491800 & 0.18584200 \\
\hline $\mathrm{C}$ & 7.94872200 & 8.76636000 & 0.87227600 \\
\hline $\mathrm{C}$ & 9.97662100 & 6.61113400 & 3.97204200 \\
\hline $\mathrm{C}$ & 9.19044700 & 8.72519300 & 2.96389600 \\
\hline $\mathrm{H}$ & 7.48529000 & 9.31526100 & 0.05035200 \\
\hline $\mathrm{C}$ & 8.57646200 & 9.43885900 & 1.90058900 \\
\hline C & 10.53919400 & 7.36256300 & 5.00196100 \\
\hline $\mathrm{C}$ & 10.30683700 & 5.17488400 & 3.93148000 \\
\hline $\mathrm{C}$ & 9.80444000 & 9.43886600 & 4.02720400 \\
\hline $\mathrm{H}$ & 8.60883700 & 10.53052100 & 1.92170300 \\
\hline
\end{tabular}




\begin{tabular}{|c|c|c|c|}
\hline $\mathrm{H}$ & 11.14552700 & 6.83488600 & 5.74194800 \\
\hline $\mathrm{C}$ & 10.43219700 & 8.76636200 & 5.05552000 \\
\hline V & 12.43374700 & 4.49184000 & 4.33935400 \\
\hline $\mathrm{C}$ & 10.76823100 & 4.42688000 & 2.80265600 \\
\hline $\mathrm{C}$ & 10.30302700 & 4.28851400 & 5.05852300 \\
\hline $\mathrm{H}$ & 9.77205500 & 10.53050100 & 4.00609600 \\
\hline $\mathrm{H}$ & 10.89563400 & 9.31526500 & 5.87740900 \\
\hline $\mathrm{C}$ & 11.02224100 & 3.08999300 & 3.22279600 \\
\hline $\mathrm{C}$ & 10.73631500 & 3.00274300 & 4.61859500 \\
\hline $\mathrm{H}$ & 9.99852200 & 4.56203400 & 6.06543500 \\
\hline $\mathrm{C}$ & 14.44165400 & 5.19870100 & 3.54167100 \\
\hline $\mathrm{C}$ & 13.92320600 & 6.20102500 & 4.41631500 \\
\hline $\mathrm{H}$ & 13.61739700 & 7.20437500 & 4.13075900 \\
\hline $\mathrm{C}$ & 13.82391000 & 5.63589000 & 5.72409200 \\
\hline $\mathrm{H}$ & 13.45560600 & 6.14234600 & 6.61272500 \\
\hline $\mathrm{C}$ & 14.28174400 & 4.28395200 & 5.65802600 \\
\hline C & 14.66316900 & 4.01378700 & 4.30889500 \\
\hline $\mathrm{H}$ & 10.88816900 & 4.82399100 & 1.79963300 \\
\hline $\mathrm{H}$ & 11.38862400 & 2.28301300 & 2.59371100 \\
\hline $\mathrm{H}$ & 10.83914500 & 2.11663100 & 5.23959300 \\
\hline $\mathrm{H}$ & 14.61776200 & 5.31189900 & 2.47498600 \\
\hline $\mathrm{H}$ & 14.32139400 & 3.58155900 & 6.48680900 \\
\hline $\mathrm{H}$ & 15.04238800 & 3.06826600 & 3.92968700 \\
\hline
\end{tabular}

$\left[\mathbf{V}_{2} \mathbf{O}_{2}(\mu-\mathrm{O}-\mathbf{M e})_{2}\left(\mathbf{m a}_{2}\right)\right]$ (high-spin structure)

$E_{\text {tot }}=-3184.59458873476$ a.u.

C $\quad-0.205242 \quad 0.220268 \quad-0.090306$

$\begin{array}{llll}\text { C } & -0.060745 & -0.168180 & 1.232389\end{array}$

$\begin{array}{llll}0 & 1.194869 & -0.323257 & 1.771559\end{array}$ 

$\begin{array}{llll}\text { C } & 2.299550 & -0.109527 & 1.033471\end{array}$
$\begin{array}{llll}\text { C } & 2.253789 & 0.262766 & -0.279011\end{array}$
$\begin{array}{llll}\text { C } & 0.982610 & 0.435861 & -0.894077\end{array}$
$\begin{array}{llll}\text { H } & 3.206070 & -0.274255 & 1.611875\end{array}$
$\begin{array}{llll}\mathrm{H} & 3.172759 & 0.422688 & -0.841055\end{array}$
$\begin{array}{llll}0 & 0.795666 & 0.781149 & -2.118037\end{array}$
$\begin{array}{llll}0 & -1.359309 & 0.421190 & -0.692155\end{array}$
$\begin{array}{llll}\mathrm{V} & -1.194235 & 0.817347 & -2.646509\end{array}$
$\begin{array}{llll}0 & -1.460408 & -0.505220 & -3.512395\end{array}$
$\begin{array}{llll}0 & -0.832072 & 2.377849 & -3.811165\end{array}$
$\begin{array}{llll}0 & -2.869714 & 1.878516 & -2.589842\end{array}$
$\begin{array}{llll}\mathrm{V} & -2.412363 & 3.541778 & -3.558110\end{array}$
$\begin{array}{llll}0 & -2.056511 & 4.704987 & -2.513211\end{array}$
$\begin{array}{llll}0 & -2.218737 & 4.239095 & -5.426258\end{array}$
$\begin{array}{llll}0 & -4.393563 & 3.804034 & -4.052257\end{array}$
$\begin{array}{llll}\text { C } & -4.551151 & 4.394118 & -5.183910\end{array}$
C $\quad-3.350454 \quad 4.654502 \quad-5.955004$
$\begin{array}{llll}\text { C } & -5.802356 & 4.795836 & -5.728160\end{array}$
$\begin{array}{llll}\text { C } & -5.816711 & 5.425079 & -6.939541\end{array}$
$\begin{array}{llll}0 & -4.699163 & 5.678380 & -7.644888\end{array}$
$\begin{array}{llll}\text { C } & -3.462489 & 5.303025 & -7.175191\end{array}$
$\begin{array}{llll}\mathrm{H} & -6.730671 & 4.613047 & -5.188939\end{array}$
$\mathrm{H} \quad-6.705313 \quad 5.784118 \quad-7.454236$
$\begin{array}{llll}\text { C } & -2.339487 & 5.657680 & -8.079959\end{array}$
$\begin{array}{llll}\mathrm{H} & -2.477797 & 5.218709 & -9.079962\end{array}$
$\mathrm{H} \quad-2.254146 \quad 6.748927 \quad-8.201862$
$\mathrm{H} \quad-1.406050 \quad 5.277896 \quad-7.649773$
$\begin{array}{llll}\text { C } & -1.165303 & -0.437583 & 2.187586\end{array}$
$\begin{array}{llll}\mathrm{H} & -1.140718 & 0.264343 & 3.035919\end{array}$ 


$\begin{array}{lrrr}\mathrm{H} & -2.120742 & -0.324315 & 1.662907 \\ \mathrm{H} & -1.099653 & -1.458013 & 2.595164 \\ \mathrm{C} & 0.339634 & 2.630179 & -4.588527 \\ \mathrm{H} & 0.199990 & 3.558869 & -5.158749 \\ \mathrm{H} & 1.214539 & 2.724842 & -3.928159 \\ \mathrm{H} & 0.505973 & 1.798040 & -5.290153 \\ \mathrm{C} & -4.008783 & 1.625472 & -1.766384 \\ \mathrm{H} & -4.822155 & 2.304695 & -2.058300 \\ \mathrm{H} & -4.338156 & 0.584601 & -1.906898 \\ \mathrm{H} & -3.753974 & 1.782609 & -0.707257\end{array}$

$\left[\mathbf{V}_{2} \mathrm{O}_{2}(\mu-\mathrm{O}-\mathbf{M e})_{2}\left(\mathbf{m a}_{2}\right)\right]$ (BS structure)

$E_{\mathrm{tot}}=-3184.59593625789$ a.u.
C $\quad-0.213111 \quad 0.233307 \quad-0.098311$
$\begin{array}{llll}\text { C } & -0.068166 & -0.163058 & 1.222092\end{array}$
$\begin{array}{llll}0 & 1.188001 & -0.325323 & 1.758528\end{array}$
$\begin{array}{llll}\text { C } & 2.292438 & -0.112013 & 1.020011\end{array}$
$\begin{array}{llll}\text { C } & 2.246169 & 0.268129 & -0.290223\end{array}$
$\begin{array}{llll}\text { C } & 0.974551 & 0.449916 & -0.902024\end{array}$
H $3.199204 \quad-0.284044 \quad 1.596044$
$\begin{array}{llll}\text { H } & 3.164792 & 0.427097 & -0.853205\end{array}$
$\begin{array}{llll}0 & 0.786136 & 0.802956 & -2.123143\end{array}$
$\begin{array}{llll}0 & -1.366112 & 0.440754 & -0.699113\end{array}$
$\begin{array}{llll}\mathrm{V} & -1.205536 & 0.851265 & -2.653473\end{array}$
$\begin{array}{llll}0 & -1.470478 & -0.468986 & -3.522701\end{array}$
$\begin{array}{llll}0 & -0.807451 & 2.391485 & -3.821954\end{array}$
$\begin{array}{llll}0 & -2.890000 & 1.880728 & -2.566193\end{array}$
$\begin{array}{llll}\mathrm{V} & -2.404067 & 3.518125 & -3.554217\end{array}$
$\begin{array}{llll}0 & -2.056024 & 4.693508 & -2.521205\end{array}$ 

$\begin{array}{llll}0 & -2.213446 \quad 4.213376 & -5.425506\end{array}$
$\begin{array}{llll}0 & -4.386498 & 3.781629 & -4.050164\end{array}$
$\begin{array}{llll}\text { C } & -4.544188 \quad 4.376995 & -5.178695\end{array}$
C $\quad-3.343854 \quad 4.635796 \quad-5.950810$
$\begin{array}{llll}\text { C } & -5.794877 & 4.786531 & -5.718245\end{array}$
C $\quad-5.809345 \quad 5.420243 \quad-6.927234$
$\begin{array}{llll}0 & -4.692135 & 5.672524 & -7.633408\end{array}$
$\begin{array}{llll}\text { C } & -3.455591 & 5.291966 & -7.166958\end{array}$
$\mathrm{H} \quad-6.722590 \quad 4.605100 \quad-5.177475$
$\mathrm{H} \quad-6.697700 \quad 5.784675 \quad-7.438706$
C $\quad-2.333842 \quad 5.647830 \quad-8.072800$
$\mathrm{H} \quad-2.470009 \quad 5.204266 \quad-9.071130$
$\mathrm{H} \quad-2.254009 \quad 6.738871 \quad-8.199074$
$\mathrm{H} \quad-1.398334 \quad 5.273847 \quad-7.642061$
$\begin{array}{llll}\text { C } & -1.171026 & -0.434859 & 2.178702\end{array}$
$\mathrm{H} \quad-1.145604 \quad 0.265570 \quad 3.028335$
$\mathrm{H} \quad-2.128079-0.321743 \quad 1.657032$
$\mathrm{H} \quad-1.103050 \quad-1.455808 \quad 2.584532$
C $\quad 0.363735 \quad 2.642186 \quad-4.598511$
H $\quad 0.219705 \quad 3.565783 \quad-5.175589$
$\mathrm{H} \quad 1.236313 \quad 2.745324-3.936506$
$\mathrm{H} \quad 0.533933 \quad 1.804192 \quad-5.291965$
C $\quad-4.029486 \quad 1.623019 \quad-1.746828$
$\mathrm{H} \quad-4.842641 \quad 2.302500 \quad-2.038362$
$\mathrm{H} \quad-4.355387 \quad 0.581708 \quad-1.891602$
$\mathrm{H} \quad-3.775063 \quad 1.776774 \quad-0.687255$

\section{para- $\mathrm{C}_{8} \mathrm{H}_{8}$}

$E_{\text {tot }}=-309.563408178$ a.u. 


$\begin{array}{lrrr}\text { C } & 0.000000 & 0.000000 & 0.000000 \\ \text { C } & 0.000000 & 0.000000 & 1.400000 \\ \text { C } & 1.212436 & 0.000000 & 2.100000 \\ \text { C } & 2.424871 & 0.000000 & 1.400000 \\ \text { C } & 2.424871 & 0.000000 & 0.000000 \\ \text { C } & 1.212436 & 0.000000 & -0.700000 \\ \text { H } & -0.943102 & 0.000000 & 1.944500 \\ \text { H } & 1.212436 & 0.000000 & 3.189000 \\ \text { H } & 3.367973 & 0.000000 & -0.544500 \\ \text { H } & 1.212436 & 0.000000 & -1.789000 \\ \text { C } & -1.558846 & 0.000000 & -0.900000 \\ \text { C } & 3.983717 & 0.000000 & 2.300000 \\ \text { H } & 4.926819 & 0.000000 & 1.755500 \\ \text { H } & 3.983717 & 0.000000 & 3.389000 \\ \text { H } & -1.359853 & 0.000000 & -1.970665 \\ \text { H } & -2.386572 & 0.000000 & -0.192335\end{array}$

\subsection{Delocalized spins}

$\left[\mathrm{Cu}_{2}(\mathrm{Ac})_{4}\left(\mathrm{H}_{2} \mathrm{O}\right)_{2}\right]$ (high-spin structure)

$E_{\mathrm{tot}}=-4349.06350393134$ a.u.

$\begin{array}{lrrr}\text { CU } & -0.03266400 & 0.16599600 & -0.07180300 \\ \text { CU } & -0.11272700 & 0.00670400 & -2.57950700 \\ \mathrm{O} & 0.14970300 & -0.37814800 & 2.18576000 \\ \mathrm{H} & 1.02869400 & -0.78516300 & 2.06736600 \\ \mathrm{H} & -0.47253700 & -1.12340500 & 2.08279700 \\ \mathrm{O} & 1.00525700 & 1.85611500 & -0.30982200 \\ \mathrm{O} & -1.74526200 & 1.17412000 & -0.19829600\end{array}$




\begin{tabular}{|c|c|c|c|}
\hline 0 & -1.06782900 & -1.58459400 & -0.08659800 \\
\hline 0 & 1.69529800 & -0.90223500 & -0.17775600 \\
\hline 0 & -1.07201800 & -1.73074900 & -2.35154000 \\
\hline 0 & -1.83888700 & 0.99918100 & -2.46345200 \\
\hline 0 & 1.67217000 & -0.97224000 & -2.44272800 \\
\hline 0 & 0.89688200 & 1.76801300 & -2.57571100 \\
\hline C & -2.29249900 & 1.37458900 & -1.33269500 \\
\hline $\mathrm{C}$ & -1.35928800 & -2.16073900 & -1.18917500 \\
\hline $\mathrm{C}$ & 2.18845000 & -1.23093700 & -1.30675900 \\
\hline $\mathrm{C}$ & 1.24324800 & 2.31294500 & -1.47362800 \\
\hline $\mathrm{C}$ & -2.11179700 & -3.47451900 & -1.09687900 \\
\hline C & -3.61163400 & 2.12279400 & -1.32589200 \\
\hline $\mathrm{C}$ & 2.00284300 & 3.62393700 & -1.54483700 \\
\hline $\mathrm{C}$ & 3.49881200 & -1.99550200 & -1.28409200 \\
\hline $\mathrm{H}$ & -2.35594900 & -3.85397400 & -2.09421400 \\
\hline $\mathrm{H}$ & -1.49536800 & -4.20992500 & -0.56106000 \\
\hline $\mathrm{H}$ & -3.03168000 & -3.32904800 & -0.51428000 \\
\hline $\mathrm{H}$ & -3.93421800 & 2.35075700 & -2.34695300 \\
\hline $\mathrm{H}$ & -4.37166400 & 1.50282000 & -0.82970100 \\
\hline $\mathrm{H}$ & -3.50890800 & 3.04676400 & -0.74190400 \\
\hline $\mathrm{H}$ & 2.19877600 & 3.90985700 & -2.58348900 \\
\hline $\mathrm{H}$ & 1.41306300 & 4.40748500 & -1.04941800 \\
\hline $\mathrm{H}$ & 2.94838500 & 3.52981700 & -0.99405400 \\
\hline $\mathrm{H}$ & 3.84089100 & -2.22011700 & -2.29955500 \\
\hline $\mathrm{H}$ & 4.25824300 & -1.40316600 & -0.75560600 \\
\hline $\mathrm{H}$ & 3.36426100 & -2.93108400 & -0.72365800 \\
\hline 0 & 0.47452700 & 0.05692300 & -4.82969300 \\
\hline $\mathrm{H}$ & 1.25431200 & -0.51258600 & -4.68848300 \\
\hline $\mathrm{H}$ & 0.82758700 & 0.96441700 & -4.76390600 \\
\hline
\end{tabular}


$\left[\mathbf{C u}_{2}(\mathbf{A c})_{4}\left(\mathbf{H}_{2} \mathbf{O}\right)_{2}\right]$ (BS structure) $E_{\text {tot }}=-4349.06603531730$ a.u.
CU $\quad-0.032556 \quad 0.166587 \quad-0.064384$
$\begin{array}{llll}\text { CU } & -0.112933 & 0.005696 & -2.585827\end{array}$
$\begin{array}{llll}0 & 0.150870 & -0.380098 & 2.190394\end{array}$
$\mathrm{H} \quad 1.030308 \quad-0.785755 \quad 2.070867$
$\mathrm{H} \quad-0.470811-1.125695 \quad 2.086411$
$\begin{array}{llll}0 & 1.003006 & 1.856333 & -0.307795\end{array}$
$\begin{array}{llll}0 & -1.745233 & 1.173267 & -0.196799\end{array}$
$\begin{array}{llll}0 & -1.069043 & -1.581851 & -0.085271\end{array}$
$\begin{array}{llll}0 & 1.693814 & -0.901583 & -0.175867\end{array}$
$\begin{array}{llll}0 & -1.072599 & -1.728688 & -2.353137\end{array}$
$\begin{array}{llll}0 & -1.837879 & 0.997812 & -2.465478\end{array}$
$\begin{array}{llll}0 & 1.670015 & -0.970672 & -2.443298\end{array}$
$\begin{array}{llll}0 & 0.893941 & 1.766846 & -2.576508\end{array}$
$\begin{array}{llll}\text { C } & -2.286695 & 1.370899 & -1.333310\end{array}$
C $\quad-1.357279-2.154376 \quad-1.189592$
C $\quad 2.183089-1.227998-1.306405$
$\begin{array}{llll}\text { C } & 1.238840 & 2.307832 & -1.473134\end{array}$
C $\quad-2.109764-3.470455-1.097452$
$\begin{array}{llll}\text { C } & -3.607651 & 2.120861 & -1.326912\end{array}$
$\begin{array}{llll}\text { C } & 2.000644 & 3.619949 & -1.545338\end{array}$
$\begin{array}{llll}\text { C } & 3.494930 & -1.993514 & -1.284546\end{array}$
$\mathrm{H} \quad-2.353713 \quad-3.849748 \quad-2.094453$
$\mathrm{H} \quad-1.492106 \quad-4.204195 \quad-0.561187$
$\begin{array}{llll}\mathrm{H} & -3.028987 & -3.324481 & -0.514417\end{array}$
$\mathrm{H} \quad-3.930228 \quad 2.349219 \quad-2.347414$
$\mathrm{H} \quad-4.365838 \quad 1.499288 \quad-0.830391$
$\begin{array}{llll}\mathrm{H} & -3.503258 & 3.043490 & -0.741615\end{array}$ 


$\begin{array}{lrrr}\text { H } & 2.197229 & 3.905332 & -2.583589 \\ \text { H } & 1.410210 & 4.402814 & -1.050070 \\ \text { H } & 2.945227 & 3.524895 & -0.993586 \\ \text { H } & 3.837056 & -2.218363 & -2.299583 \\ \text { H } & 4.253480 & -1.400402 & -0.756070 \\ \text { H } & 3.360373 & -2.928570 & -0.723608 \\ \mathrm{O} & 0.475933 & 0.057583 & -4.833800 \\ \mathrm{H} & 1.255342 & -0.512202 & -4.691727 \\ \mathrm{H} & 0.828739 & 0.965047 & -4.766332\end{array}$

$\left[\mathbf{C o}_{2}\left(\eta^{5}-\mathbf{C p}\right)_{2}\left(\mu-\eta^{5}-1,8-\mathbf{D C N}\right)\right]$ (high-spin structure $E_{\text {tot }}=-3924.16738828946$ a.u.
C $\quad 0.428949 \quad-0.876513 \quad-0.462974$
$\begin{array}{llll}\text { C } & 0.035880 & -0.281655 & 0.773338\end{array}$
C $\quad 1.850111-1.133562 \quad-0.434131$
$\begin{array}{llll}\text { C } & 1.216231 & -0.304479 & 1.613416\end{array}$
$\begin{array}{llll}\text { C } & 2.342825 & -0.737979 & 0.842552\end{array}$
CO $\quad 0.947855 \quad-2.293005 \quad 1.021965$
$\begin{array}{llll}\text { C } & -0.392445 & -3.576370 & 2.051647\end{array}$
$\begin{array}{llll}\text { C } & 1.267555 & -4.343356 & 0.621604\end{array}$
$\begin{array}{llll}\text { C } & -0.148819 & -4.072530 & 0.743252\end{array}$
$\begin{array}{llll}\text { C } & 0.878761 & -3.497479 & 2.723981\end{array}$
$\begin{array}{llll}\text { C } & 1.889600 & -4.039638 & 1.863077\end{array}$
$\begin{array}{llll}\text { C } & -1.316191 & 0.117929 & 1.184927\end{array}$
$\begin{array}{lrrr}\text { C } & -1.553458 & 1.233921 & 2.071029\end{array}$
$\begin{array}{llll}\text { C } & -2.399185 & -0.679894 & 0.801377\end{array}$
$\begin{array}{llll}\text { C } & -0.598277 & 2.285798 & 2.336012\end{array}$
$\begin{array}{llll}\text { C } & -0.832484 & 3.152862 & 3.408375\end{array}$
$\begin{array}{llll}\text { C } & 0.536723 & 2.561939 & 1.445124\end{array}$ 


\begin{tabular}{|c|c|c|c|}
\hline C & 1.809191 & 3.101974 & 1.800566 \\
\hline C & 0.520104 & 2.515152 & -0.003198 \\
\hline C & 1.817816 & 2.851780 & -0.506922 \\
\hline $\mathrm{C}$ & 2.607483 & 3.257911 & 0.607183 \\
\hline $\mathrm{CO}$ & 0.962895 & 4.505369 & 0.458425 \\
\hline C & -0.633880 & 5.850935 & 0.813367 \\
\hline C & 0.539042 & 6.318335 & 1.460354 \\
\hline C & 1.552019 & 6.529653 & 0.450113 \\
\hline $\mathrm{C}$ & 0.985927 & 6.238394 & -0.823583 \\
\hline $\mathrm{C}$ & -0.340572 & 5.751517 & -0.595630 \\
\hline $\mathrm{C}$ & -3.686561 & -0.495220 & 1.337362 \\
\hline C & -3.888204 & 0.441665 & 2.332533 \\
\hline $\mathrm{C}$ & -2.834078 & 1.304075 & 2.738663 \\
\hline $\mathrm{C}$ & -3.029336 & 2.235011 & 3.794893 \\
\hline $\mathrm{C}$ & -2.019503 & 3.104074 & 4.161374 \\
\hline $\mathrm{H}$ & -3.992811 & 2.248745 & 4.308815 \\
\hline $\mathrm{H}$ & -4.859010 & 0.534808 & 2.824162 \\
\hline $\mathrm{H}$ & -4.504400 & -1.142934 & 1.015564 \\
\hline $\mathrm{H}$ & -2.214826 & -1.521396 & 0.131753 \\
\hline $\mathrm{H}$ & -0.107932 & 3.944325 & 3.604661 \\
\hline $\mathrm{H}$ & -2.165604 & 3.804685 & 4.985691 \\
\hline $\mathrm{H}$ & -0.890875 & -4.229001 & -0.035162 \\
\hline $\mathrm{H}$ & 1.762386 & -4.731083 & -0.265073 \\
\hline $\mathrm{H}$ & 2.948530 & -4.121267 & 2.090276 \\
\hline $\mathrm{H}$ & 1.035656 & -3.132797 & 3.736001 \\
\hline $\mathrm{H}$ & -1.348933 & -3.255961 & 2.453690 \\
\hline $\mathrm{H}$ & 3.368337 & -0.812964 & 1.191704 \\
\hline $\mathrm{H}$ & 1.243009 & 0.016684 & 2.650381 \\
\hline & -0.223663 & -1.053547 & -1.314372 \\
\hline
\end{tabular}




$\begin{array}{lrrr}\mathrm{H} & 2.436279 & -1.533819 & -1.257193 \\ \mathrm{H} & 2.119512 & 2.858355 & -1.550093 \\ \mathrm{H} & 3.638864 & 3.599197 & 0.577135 \\ \mathrm{H} & -0.334474 & 2.214814 & -0.602132 \\ \mathrm{H} & 2.146973 & 3.309997 & 2.813011 \\ \mathrm{H} & 1.494569 & 6.291249 & -1.781638 \\ \mathrm{H} & 2.563909 & 6.880816 & 0.634706 \\ \mathrm{H} & -1.031073 & 5.404419 & -1.360334 \\ \mathrm{H} & -1.565796 & 5.565993 & 1.293011 \\ \mathrm{H} & 0.664832 & 6.482118 & 2.527156\end{array}$

$\left[\mathbf{C o}_{2}\left(\eta^{5}-\mathbf{C p}\right)_{2}\left(\mu-\eta^{5}-1,8-\mathbf{D C N}\right)\right]$ (BS structure) $E_{\text {tot }}=-3924.16731173741$ a.u.
$\begin{array}{llll}\text { C } & 0.414084 & -0.927313 & -0.461499\end{array}$
$\begin{array}{llll}\text { C } & 0.037113 & -0.292268 & 0.769629\end{array}$
$\begin{array}{llll}\text { C } & 1.839127 & -1.158899 & -0.467439\end{array}$
$\begin{array}{llll}\text { C } & 1.220956 & -0.322820 & 1.588579\end{array}$
$\begin{array}{llll}\text { C } & 2.341175 & -0.773958 & 0.804308\end{array}$
$\begin{array}{llll}\text { CO } & 0.946129 & -2.312719 & 1.014981\end{array}$
$\begin{array}{llll}\text { C } & -0.399486 & -3.583197 & 2.066938\end{array}$
$\begin{array}{llll}\text { C } & 1.260394 & -4.385618 & 0.637704\end{array}$
$\begin{array}{llll}\text { C } & -0.149607 & -4.073754 & 0.753107\end{array}$
$\begin{array}{llll}\text { C } & 0.870059 & -3.489298 & 2.725203\end{array}$
$\begin{array}{llll}\text { C } & 1.883494 & -4.047163 & 1.861831\end{array}$
$\begin{array}{llll}\text { C } & -1.310525 & 0.112095 & 1.184211\end{array}$
$\begin{array}{llll}\text { C } & -1.551358 & 1.230008 & 2.068594\end{array}$
$\begin{array}{llll}\text { C } & -2.393709 & -0.687845 & 0.799387\end{array}$
$\begin{array}{llll}\text { C } & -0.603121 & 2.289941 & 2.332516\end{array}$
$\begin{array}{llll}\text { C } & -0.842803 & 3.154669 & 3.407894\end{array}$ 


\begin{tabular}{|c|c|c|c|}
\hline C & 0.530939 & 2.577513 & 1.447364 \\
\hline C & 1.793917 & 3.154608 & 1.811867 \\
\hline C & 0.534173 & 2.539586 & 0.007701 \\
\hline $\mathrm{C}$ & 1.841801 & 2.889004 & -0.483447 \\
\hline C & 2.622287 & 3.281997 & 0.636665 \\
\hline $\mathrm{CO}$ & 0.967613 & 4.530396 & 0.465146 \\
\hline C & -0.634821 & 5.883263 & 0.818450 \\
\hline C & 0.555854 & 6.332663 & 1.451674 \\
\hline C & 1.557109 & 6.570101 & 0.431032 \\
\hline $\mathrm{C}$ & 0.989387 & 6.248446 & -0.825568 \\
\hline $\mathrm{C}$ & -0.342445 & 5.754841 & -0.581897 \\
\hline $\mathrm{C}$ & -3.680361 & -0.508037 & 1.335952 \\
\hline $\mathrm{C}$ & -3.884547 & 0.427675 & 2.332019 \\
\hline $\mathrm{C}$ & -2.833211 & 1.293948 & 2.737385 \\
\hline $\mathrm{C}$ & -3.033526 & 2.223185 & 3.794157 \\
\hline $\mathrm{C}$ & -2.028621 & 3.098286 & 4.160628 \\
\hline $\mathrm{H}$ & -3.996788 & 2.230534 & 4.308612 \\
\hline $\mathrm{H}$ & -4.855417 & 0.517441 & 2.824107 \\
\hline $\mathrm{H}$ & -4.496235 & -1.158067 & 1.013645 \\
\hline $\mathrm{H}$ & -2.208384 & -1.527774 & 0.128269 \\
\hline $\mathrm{H}$ & -0.123392 & 3.950140 & 3.606344 \\
\hline $\mathrm{H}$ & -2.178608 & 3.797141 & 4.985870 \\
\hline $\mathrm{H}$ & -0.893409 & -4.230068 & -0.024168 \\
\hline $\mathrm{H}$ & 1.749184 & -4.790013 & -0.244486 \\
\hline $\mathrm{H}$ & 2.941787 & -4.129870 & 2.092601 \\
\hline $\mathrm{H}$ & 1.035531 & -3.114181 & 3.732044 \\
\hline $\mathrm{H}$ & -1.355425 & -3.254490 & 2.462817 \\
\hline $\mathrm{H}$ & 3.370128 & -0.846740 & 1.144142 \\
\hline H & 1.268417 & 0.008998 & 2.621709 \\
\hline
\end{tabular}




$\begin{array}{rrrr}\mathrm{H} & -0.252165 & -1.117203 & -1.299694 \\ \mathrm{H} & 2.412507 & -1.565501 & -1.296044 \\ \mathrm{H} & 2.153236 & 2.893097 & -1.523899 \\ \mathrm{H} & 3.652372 & 3.627245 & 0.620434 \\ \mathrm{H} & -0.307007 & 2.230302 & -0.606059 \\ \mathrm{H} & 2.114440 & 3.374381 & 2.827661 \\ \mathrm{H} & 1.487436 & 6.292348 & -1.789808 \\ \mathrm{H} & 2.567933 & 6.927301 & 0.608161 \\ \mathrm{H} & -1.033666 & 5.399756 & -1.342424 \\ \mathrm{H} & -1.561854 & 5.603670 & 1.309826 \\ \mathrm{H} & 0.691338 & 6.500274 & 2.517060\end{array}$

meta- $\mathrm{C}_{8} \mathrm{H}_{8}$ (high-spin structure $E_{\mathrm{tot}}=-309.671132520$ a.u.

$\begin{array}{llll}\mathrm{C} & -0.036898 & 0.000000 & -0.073509 \\ \mathrm{C} & -0.053343 & 0.000000 & 1.361486 \\ \mathrm{C} & 1.167995 & 0.000000 & -0.772201 \\ \mathrm{C} & 2.388541 & 0.000000 & -0.101122 \\ \mathrm{C} & 1.199721 & 0.000000 & 2.032161 \\ \mathrm{C} & 2.437271 & 0.000000 & 1.333095 \\ \mathrm{C} & 3.662920 & 0.000000 & 2.032161 \\ \mathrm{C} & -1.263563 & 0.000000 & 2.072097 \\ \mathrm{H} & -0.987029 & 0.000000 & -0.611517 \\ \mathrm{H} & 1.155400 & 0.000000 & -1.864326 \\ \mathrm{H} & 3.326093 & 0.000000 & -0.660678 \\ \mathrm{H} & 1.212418 & 0.000000 & 3.125209 \\ \mathrm{H} & 4.608540 & 0.000000 & 1.474258 \\ \mathrm{H} & 3.700531 & 0.000000 & 3.106279 \\ \mathrm{H} & -2.220851 & 0.000000 & 1.550514\end{array}$


meta- $\mathrm{C}_{8} \mathrm{H}_{8}$ (BS structure)

$E_{\mathrm{tot}}=-309.671054200$ a.u.
$\begin{array}{llll}\text { C } & -0.036075 & 0.000000 & -0.070619\end{array}$
$\begin{array}{llll}\text { C } & -0.049289 & 0.000000 & 1.356379\end{array}$
$\begin{array}{llll}\text { C } & 1.168719 & 0.000000 & -0.771319\end{array}$
$\begin{array}{llll}\text { C } & 2.388289 & -0.000000 & -0.097296\end{array}$
$\begin{array}{llll}\text { C } & 1.199418 & -0.000000 & 2.026566\end{array}$
$\begin{array}{llll}\text { C } & 2.432334 & -0.000000 & 1.329501\end{array}$
$\begin{array}{llll}\text { C } & 3.670680 & -0.000000 & 2.020873\end{array}$
$\begin{array}{llll}\text { C } & -1.271885 & 0.000000 & 2.074318\end{array}$
H $\quad-0.985633 \quad-0.000000 \quad-0.609087$
$\begin{array}{llll}\mathrm{H} & 1.156577 & 0.000000 & -1.863005\end{array}$
H $\quad 3.325733 \quad-0.000000 \quad-0.656369$
H $\quad 1.211354-0.000000 \quad 3.119251$
$\begin{array}{llll}\mathrm{H} & 4.616190 & 0.000000 & 1.479417\end{array}$
$\begin{array}{llll}\text { H } & 3.704951 & 0.000000 & 3.110021\end{array}$
$\begin{array}{llll}\mathrm{H} & -2.228773 & 0.000000 & 1.553162\end{array}$
$\begin{array}{llll}\mathrm{H} & -1.282954 & 0.000000 & 3.163941\end{array}$ 